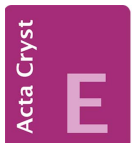

CRYSTALLOGRAPHIC COMMUNICATIONS

ISSN 2056-9890

Received 18 April 2015

Accepted 26 April 2015

Edited by H. Stoeckli-Evans, University of Neuchâtel, Switzerland

Keywords: crystal structure; 2-chloroquinolines; molecular conformation; hydrogen bonding; $\pi$ $\pi$ stacking interactions

CCDC references: 1061944; 992268; 992267; $1061945 ; 1061946$

Supporting information: this article has supporting information at journals.iucr.org/e

\section{Crystal structures of five (2-chloroquinolin-3-yl)- methyl ethers: supramolecular assembly in one and two dimensions mediated by hydrogen bonding and $\pi-\pi$ stacking}

Haliwana B. V. Sowmya, ${ }^{a}$ Tholappanavara H. Suresha Kumara, ${ }^{a, b}$ Nagendrappa Gopalpur, ${ }^{a}$ Jerry P. Jasinski, ${ }^{c} *$ Sean P. Millikan, ${ }^{\mathrm{C}}$ Hemmige S. Yathirajan ${ }^{\mathrm{d}}$ and Christopher Glidewell ${ }^{\mathbf{e}}$

${ }^{\text {a}}$ PG Department of Chemistry, Jain University, 52 Bellary Road, Hebbal, Bangalore 560024 , India, ${ }^{\mathbf{b}}$ Department of Chemistry, UBDT College of Engineering (a Constituent College of VTU, Belagavi), Davanagere 577 004, India, ${ }^{\mathbf{c}}$ Department of Chemistry, Keene State College, 229 Main Street, Keene, NH 03435-2001, USA, ${ }^{\mathbf{d} D e p a r t m e n t ~ o f ~ S t u d i e s ~}$ in Chemistry, University of Mysore, Manasagangotri, Mysore 570 006, India, and ${ }^{\mathbf{e} S c h o o l}$ of Chemistry, University of St Andrews, St Andrews, Fife KY16 9ST, Scotland. *Correspondence e-mail: jpjasinski@hotmail.com

In the molecules of the title compounds, methyl 5-bromo-2-[(2-chloroquinolin3-yl)methoxy]benzoate, $\mathrm{C}_{18} \mathrm{H}_{13} \mathrm{BrClNO}_{3}$, (I), methyl 5-bromo-2-[(2-chloro-6methylquinolin-3-yl)methoxy]benzoate, $\mathrm{C}_{19} \mathrm{H}_{15} \mathrm{BrClNO}_{3}$, (II), methyl 2-[(2chloro-6-methylquinolin-3-yl)methoxy]benzoate, $\mathrm{C}_{19} \mathrm{H}_{16} \mathrm{ClNO}_{3}$, (III), which crystallizes with $Z^{\prime}=4$ in space group $P 2_{1} 2_{1} 2_{1}$, and 2-chloro-3-[(naphthalen-1yloxy)methyl]quinoline, $\mathrm{C}_{20} \mathrm{H}_{14} \mathrm{ClNO}$, (IV), the non- $\mathrm{H}$ atoms are nearly coplanar, but in $\{5-[(2-$ chloroquinolin-3-yl)methoxy $]-4-($ hydroxymethyl)-6methylpyridin-3-yl methanol, $\mathrm{C}_{18} \mathrm{H}_{17} \mathrm{ClN}_{2} \mathrm{O}_{3}$, (V), the planes of the quinoline unit and of the unfused pyridine ring are almost parallel, although not coplanar. The molecules of (I) are linked by two independent $\pi-\pi$ stacking interactions to form chains, but there are no hydrogen bonds present in the structure. In (II), the molecules are weakly linked into chains by a single type of $\pi-\pi$ stacking interaction. In (III), three of the four independent molecules are linked by $\pi-\pi$ stacking interactions but the other molecule does not participate in such interactions. Weak $\mathrm{C}-\mathrm{H} \cdots \mathrm{O}$ hydrogen bonds link the molecules into three types of chains, two of which contain just one type of independent molecule while the third type of chain contains two types of molecule. The molecules of (IV) are linked into chains by a $\mathrm{C}-\mathrm{H} \cdots \pi$ (arene) hydrogen bond, but $\pi-\pi$ stacking interactions are absent. In (V), there is an intramolecular $\mathrm{O}-\mathrm{H} \cdots \mathrm{O}$ hydrogen bond, and molecules are linked into sheets by a combination of $\mathrm{O}-$ $\mathrm{H} \cdots \mathrm{N}$ hydrogen bonds and $\pi-\pi$ stacking interactions.

\section{Chemical context}

The quinoline nucleus occurs in a number of natural compounds, such as the Cinchona alkaloids, and many of these are pharmacologically active substances displaying a broad range of biological activity. Quinoline itself has been found to possess antimalarial, anti-bacterial, antifungal, anthelminthic, cardiotonic, anticonvulsant, anti-inflammatory and analgesic activity (Marella et al., 2013). The synthesis, reactions and biological applications of 2-chloroquinoline-3-carbaldehydes have been reviewed (Abdel-Wahab et al., 2012), and the structure of a simple reduction product (2-chloroquinolin-3yl)methanol, derived from the parent 2-chloroquinoline-3carbaldehyde, has been reported (Hathwar et al., 2010). The structures of two related esters, [(2-chloroquinolin-3-yl)methyl acetate and (2-chloro-6-methylquinolin-3-yl)methyl acetate], have also been reported recently along with a study of their radical-scavenging and antimicrobial activities 
(Tabassum et al., 2014). Here we report the structures of five related ethers, namely methyl 5-bromo-2-[(2-chloroquinolin3-yl)methoxy]benzoate, (I) (Fig. 1), methyl 5-bromo-2-[(2chloro-6-methylquinolin-3-yl)methoxy]benzoate, (II) (Fig. 2), methyl 2-[(2-chloro-6-methylquinolin-3-yl)methoxy]benzoate, (III) (Figs. 3-6), 2-chloro-3-[(naphthalen-1-yloxy)methyl]quinoline (IV) (Fig. 7) and \{5-[(2-chloroquinolin-3-yl)methoxy]-4-(hydroxymethyl)-6-methylpyridin-3-yl\}methanol, (V) (Fig. 8). Compounds (I)-(V) are all of general type $Q \mathrm{CH}_{2} \mathrm{O}$, where $Q$ represents a 2-chloroquinolin-3-yl unit, which carries a 6-methyl substituent in compounds (II) and (III), although not in compounds (I), (IV) and (V), and where $R$ represents a methoxycarbonylphenyl unit in compounds (I)-(III), a 1naphthyl unit in compound (IV), and a multiply-substituted pyridyl unit in compound (V). Compound (I)-(V) were all prepared by reaction of the corresponding chloromethyl compounds $Q \mathrm{CH}_{2} \mathrm{Cl}$ with the appropriate hydroxy compound $R \mathrm{OH}$ under basic conditions, with yields ranging from 86 to $97 \%$.<smiles>COC(=O)c1cc(Br)ccc1OCc1cc2ccccc2nc1Cl</smiles>

(I)<smiles>COC(=O)c1ccccc1OCc1cc2cc(C)ccc2nc1Cl</smiles>

(III)<smiles>COC(=O)c1cc(Br)ccc1OCc1cc2cc(C)ccc2nc1Cl</smiles>

(II)<smiles>Clc1nc2ccccc2cc1COc1cccc2ccccc12</smiles>

(IV)<smiles>Cc1ncc(CO)c(CO)c1OCc1cc2ccccc2nc1Cl</smiles>

(v)

\section{Structural commentary}

As noted above, the molecular constitutions of compounds (I)-(III) are very similar: those of compounds (I) and (II) differ only in the presence of a 6-methyl substituent in (II) which is absent from (I), while those of compounds (II) and (III) differ only in the presence of a bromo substituent in (II) which is absent from (III). Despite these close similarities, compounds (I)-(III) all crystallize in different space groups, $P 2_{1} / n$ and Pbca, respectively, for (I) and (II), both with $Z^{\prime}=1$, and $P 2_{1} 2_{1} 2_{1}$ with $Z^{\prime}=4$ for (III). A search for possible addi-

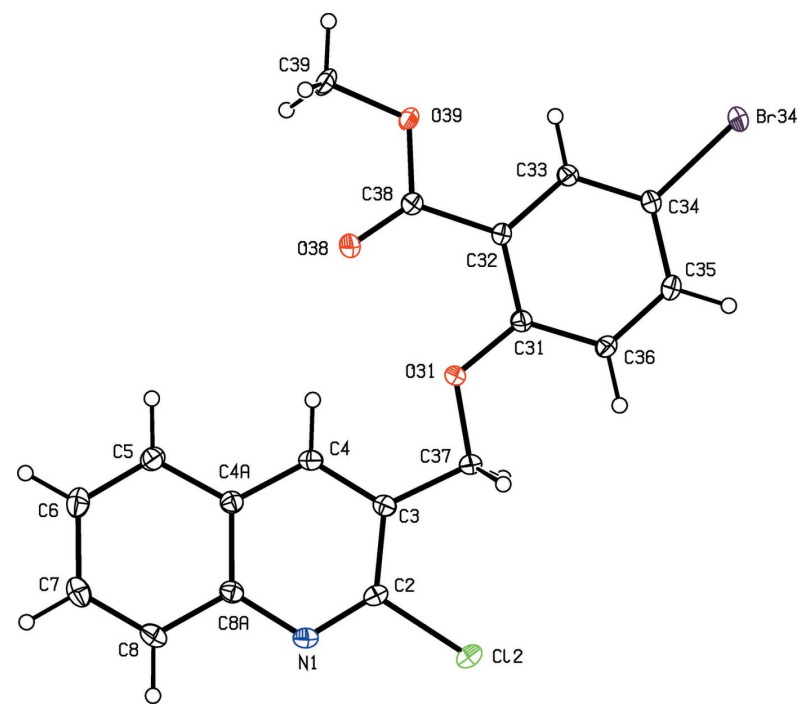

Figure 1

The molecular structure of compound (I) showing the atom-labelling scheme. Displacement ellipsoids are drawn at the $30 \%$ probability level.

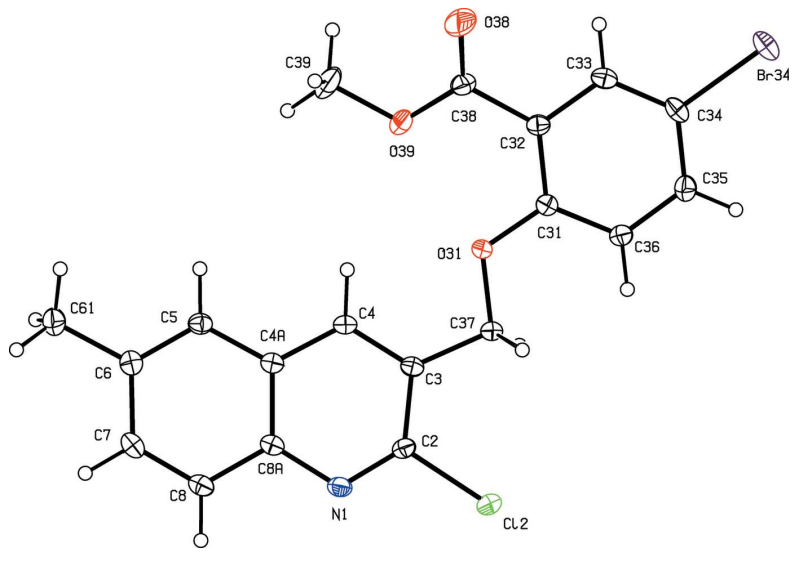

Figure 2

The molecular structure of compound (II) showing the atom-labelling scheme. Displacement ellipsoids are drawn at the $30 \%$ probability level.

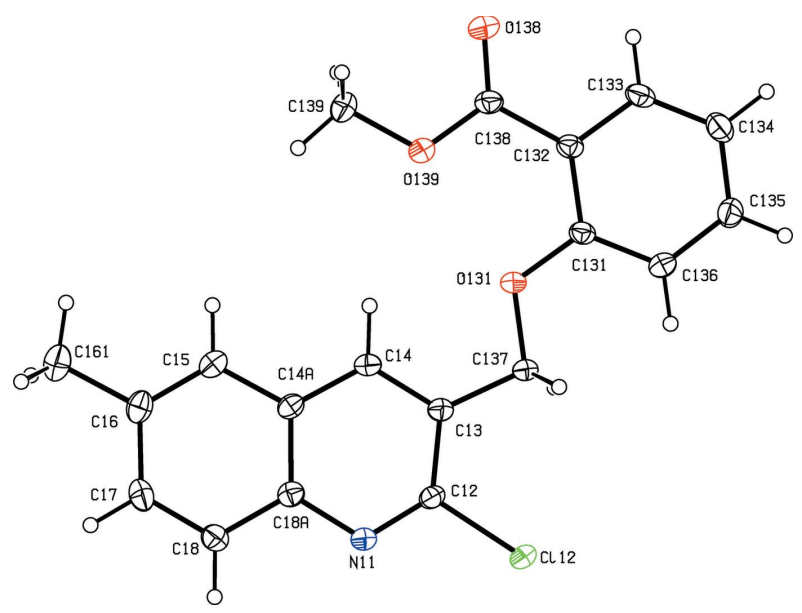

Figure 3

The structure of a type 1 molecule of compound (III), showing the atomlabelling scheme. Displacement ellipsoids are drawn at the $30 \%$ probability level. 


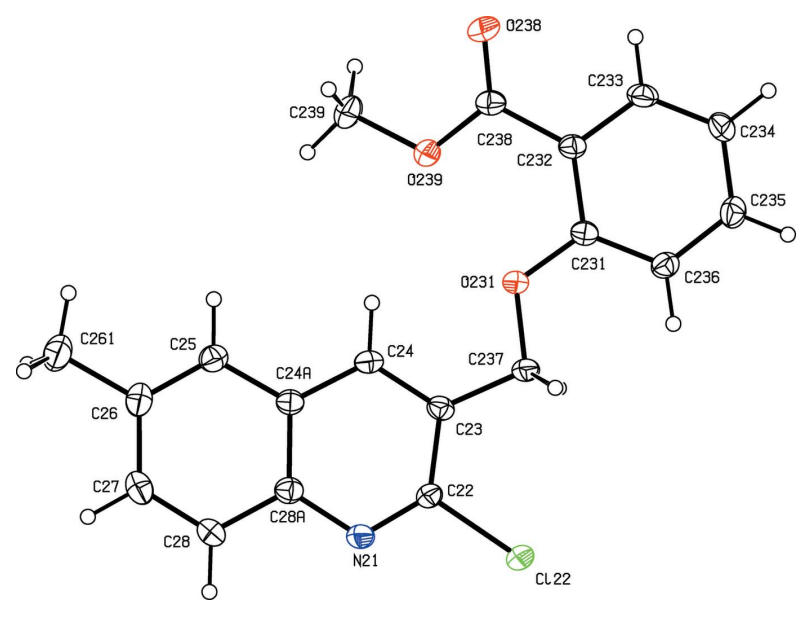

Figure 4

The structure of a type 2 molecule of compound (III), showing the atomlabelling scheme. Displacement ellipsoids are drawn at the $30 \%$ probability level.

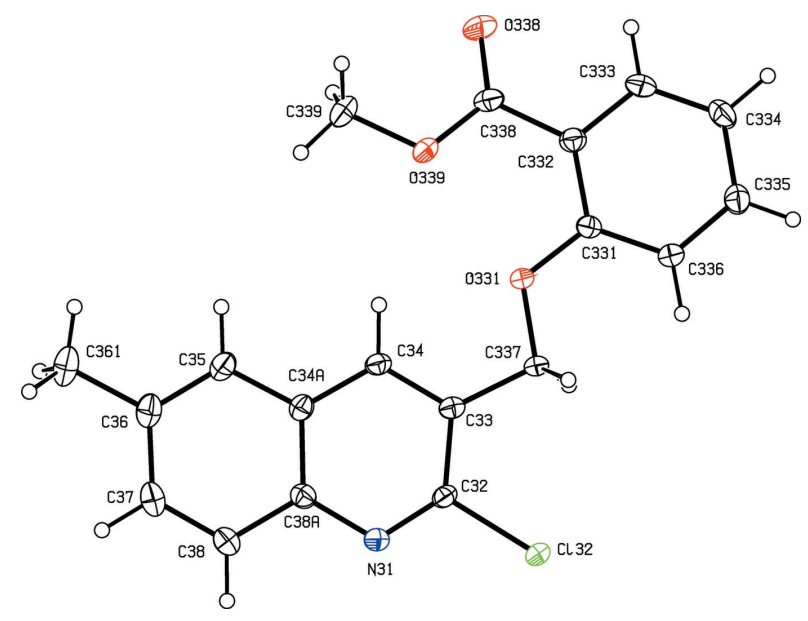

Figure 5

The structure of a type 3 molecule of compound (III), showing the atomlabelling scheme. Displacement ellipsoids are drawn at the $30 \%$ probability level.

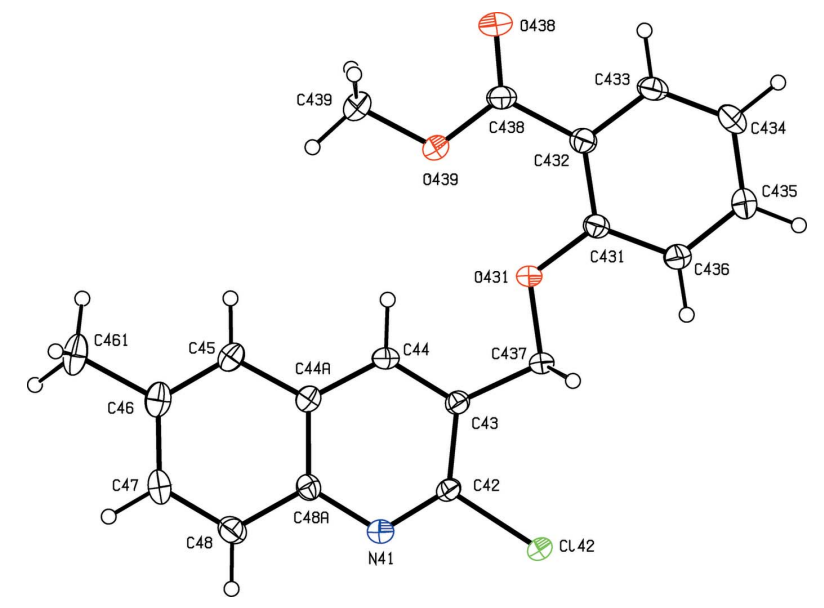

Figure 6

The structure of a type 4 molecule of compound (III), showing the atomlabelling scheme. Displacement ellipsoids are drawn at the $30 \%$ probability level.

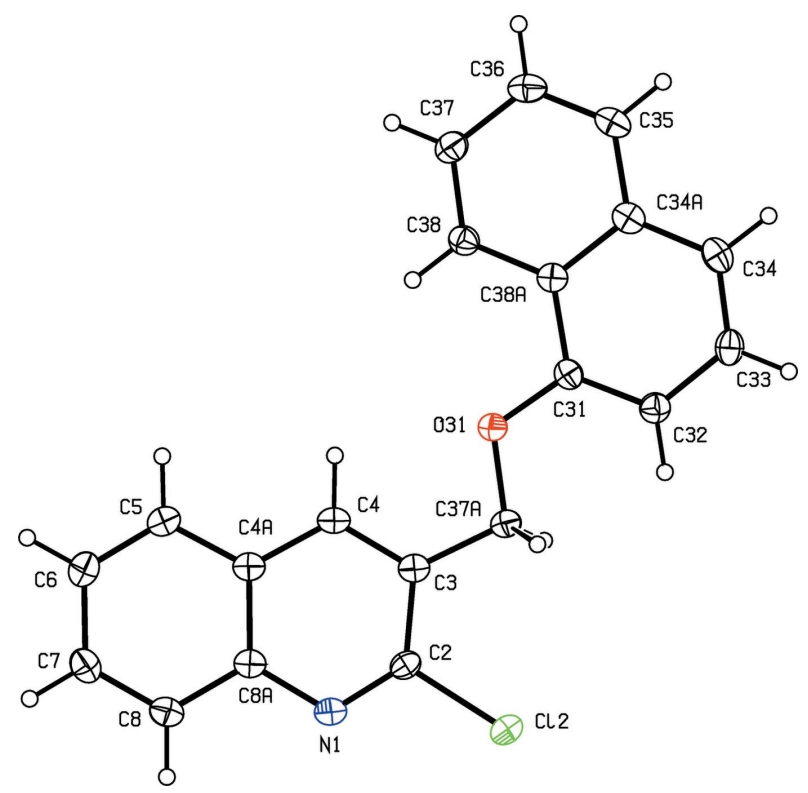

Figure 7

The molecular structure of compound (IV) showing the atom-labelling scheme. Displacement ellipsoids are drawn at the $30 \%$ probability level.

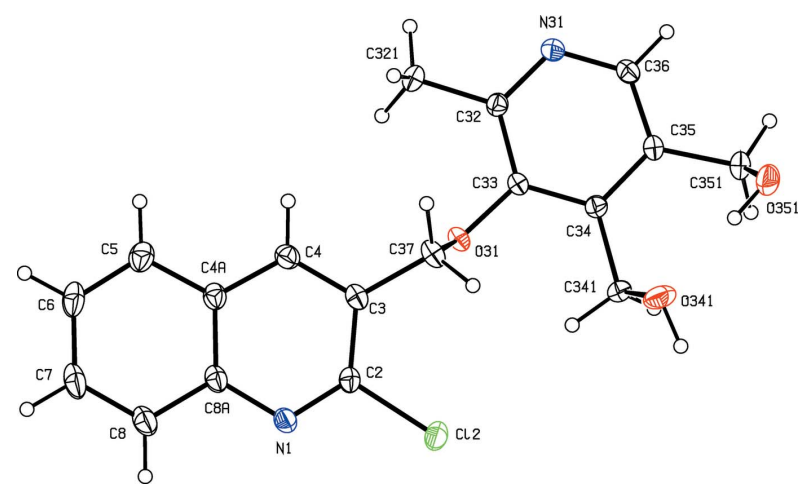

Figure 8

The molecular structure of compound (V) showing the atom-labelling scheme. Displacement ellipsoids are drawn at the $30 \%$ probability level.

tional crystallographic symmetry in compound (III) found none: comparison of the atomic coordinates for the $\mathrm{Cl}$ atoms within the selected asymmetric unit shows that while the $x$-coordinates of atoms $\mathrm{Cl} 12$ and $\mathrm{Cl} 32$ differ by $c a 0.5$ and their $z$-coordinates are almost identical, the $y$-coordinates of these two atoms differ by $\mathrm{ca} 0.13$; similarly the $x$-coordinates of atoms $\mathrm{Cl} 22$ and $\mathrm{Cl} 42$ again differ by $\mathrm{ca} 0.5$ but now the $y$-coordinates are almost identical, while the $z$-coordinates differ by $c a 0.18$. Hence it is not possible to identify even pseudosymmetry here. For compound (III), it will be convenient to refer to the molecules containing atoms N11N14 as molecules of types $1-4$, respectively. Compounds (IV) and $(\mathrm{V})$ both crystallize with $Z^{\prime}=1$, in space groups $P 2_{1}$ and $P 2_{1} / c$, respectively.

In compounds (I)-(III), the non-H atoms are almost coplanar, as shown by the relevant torsional and dihedral angles (Table 1). It is interesting to note that the orientation of the 
Table 1

Selected torsional and dihedral angles $\left(^{\circ}\right)$ for compounds (I)-(III).

'Dihedral 1' represents the dihedral angle between the mean planes of the quinoline and phenyl rings. 'Dihedral 2' represents the dihedral angle between the mean planes of the phenyl ring and the carboxyl unit.

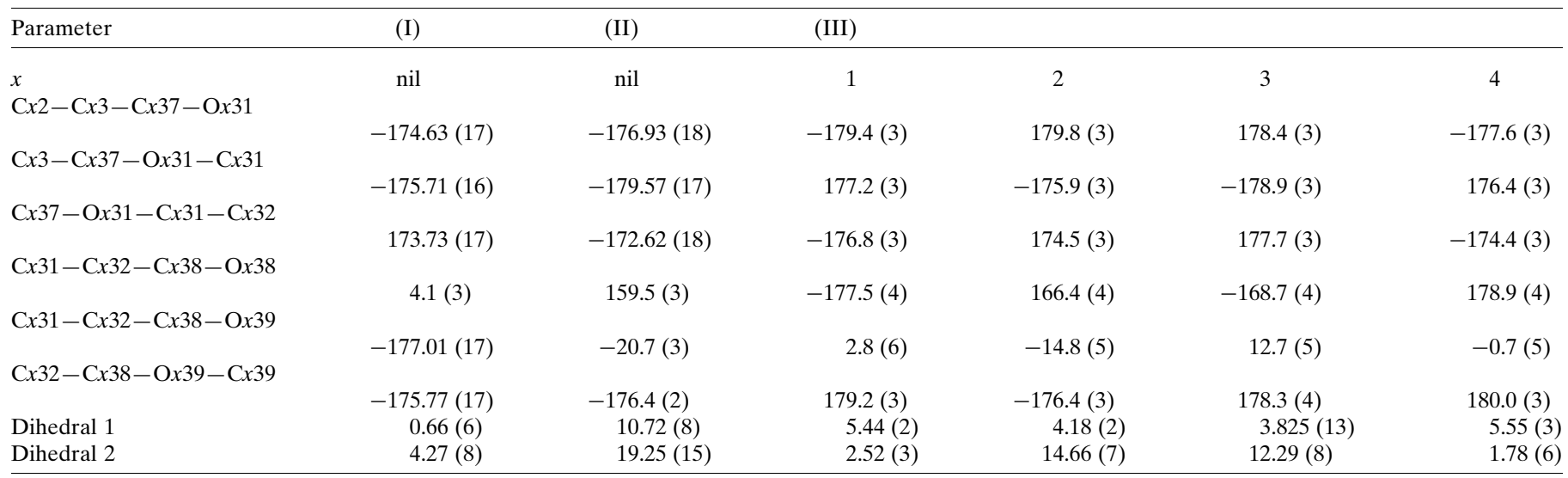

Table 2

Hydrogen bonds and short intermolecular contacts $\left(\AA{ }^{\circ}{ }^{\circ}\right)$ for compounds $(\mathrm{II})-(\mathrm{V})$.

$C g 1, C g 2$ and $C g 3$ are the centroids of rings C231-C236, C331-C336 and C31-C34,C34A,C38A, respectively.

\begin{tabular}{|c|c|c|c|c|c|}
\hline Compound & $D-\mathrm{H} \cdots A$ & $D-\mathrm{H}$ & $\mathrm{H} \cdots \mathrm{A}$ & $D \cdots A$ & $D-\mathrm{H} \cdots A$ \\
\hline (II) & $\mathrm{C} 36-\mathrm{H} 36 \cdots \mathrm{O} 38^{\mathrm{i}}$ & 0.95 & 2.53 & $3.277(3)$ & 136 \\
\hline \multirow[t]{6}{*}{ (III) } & $\mathrm{C} 28-\mathrm{H} 28 \cdots \mathrm{N} 41^{\mathrm{ii}}$ & 0.95 & 2.63 & $3.565(5)$ & 169 \\
\hline & $\mathrm{C} 136-\mathrm{H} 136 \cdots \mathrm{O} 138^{\mathrm{iii}}$ & 0.95 & 2.50 & $3.261(4)$ & 137 \\
\hline & $\mathrm{C} 336-\mathrm{H} 336 \cdots \mathrm{O} 338^{\mathrm{v}}$ & 0.95 & 2.46 & $3.238(4)$ & 139 \\
\hline & $\mathrm{C} 436-\mathrm{H} 436 \cdots \mathrm{O} 238^{\mathrm{iv}}$ & 0.95 & 2.51 & $3.254(4)$ & 136 \\
\hline & $\mathrm{C} 337-\mathrm{H} 33 B \cdots C g 1$ & 0.99 & 2.64 & $3.441(4)$ & 138 \\
\hline & $\mathrm{C} 437-\mathrm{H} 43 A \cdots C g 2$ & 0.99 & 2.64 & $3.446(4)$ & 138 \\
\hline & $\mathrm{O} 351-\mathrm{H} 351 \cdots \mathrm{O} 341$ & 0.91 & 1.86 & 2.7209 (19) & 158 \\
\hline & $\mathrm{C} 4-\mathrm{H} 4 \cdots \mathrm{O} 351^{\text {viii }}$ & 0.95 & 2.60 & $3.374(2)$ & 139 \\
\hline
\end{tabular}

Symmetry codes: (i) $x+\frac{1}{2}, y,-z+\frac{1}{2}$; (ii) $x-\frac{1}{2},-y+\frac{3}{2},-z+1$; (iii) $-x, y-\frac{1}{2},-z+\frac{1}{2}$; (iv) $-x+1, y+\frac{1}{2},-z+\frac{1}{2}$; (v) $-x+1, y-\frac{1}{2},-z+\frac{1}{2}$; (vi) $x-1, y, z$; (vii) $x-\frac{1}{2},-y+\frac{3}{2}, z+\frac{1}{2}$; (viii) $-x+\frac{1}{2}$, $y-\frac{1}{2},-z+\frac{1}{2}$.

ester function in compound (I) differs from that in compounds (II) and (III) (Table 1 and Figs. 1-6): this difference may arise, at least in part, from the participation of the carbonyl $\mathrm{O}$ atom of the ester unit in short $\mathrm{C}-\mathrm{H} \cdots \mathrm{O}$ interactions in all of the molecules of compounds (II) and (III) but not in compound (I) (Table 2). The non-H atoms in compound (IV) are also nearly coplanar, with a dihedral angle between the mean planes of the quinoline and naphthalene units of $7.39(12)^{\circ}$. By contrast, while the quinoline and pyridine units in compound (V) are nearly parallel (Fig. 8), with a dihedral angle between their mean planes of only $3.10(9)^{\circ}$, they are by no means coplanar, as indicated by the values of the torsional angles $\mathrm{C} 2-\mathrm{C} 3-\mathrm{C} 37-\mathrm{O} 31, \quad 92.08$ (18), $\mathrm{C} 3-\mathrm{C} 37-\mathrm{O} 31-\mathrm{C} 33$, 165.21 (13) and $\mathrm{C} 37-\mathrm{O} 31-\mathrm{C} 33-\mathrm{C} 32,-90.17(17)^{\circ}$. This again may perhaps be ascribed in part to the strong hydrogen bonds present in the crystal structure of (V) (Table 2).

None of the molecules of compounds (I)-(V) exhibits any internal symmetry and hence all are conformationally chiral. For compounds (I), (II) and (V), the centrosymmetric space groups accommodate equal numbers of the two conforma- tional enantiomers, but only one such enantiomer is present in each crystal of compound (IV): the absolute configuration of the enantiomer present in the crystal selected for data collection was established by means of the Flack $x$ parameter (Flack, 1983), although this has no chemical significance. For compound (III), the value of the Flack $x$ parameter gives evidence of partial inversion twinning.

\section{Supramolecular interactions}

The supramolecular assembly in compounds (I)-(V) is determined by a variety of direction-specific intermolecular interactions, including both $\pi-\pi$ stacking interactions and hydrogen bonds of $\mathrm{C}-\mathrm{H} \cdots \mathrm{N}, \mathrm{C}-\mathrm{H} \cdots \mathrm{O}$ and $\mathrm{C}-\mathrm{H} \cdots \pi$ types, as well as $\mathrm{O}-\mathrm{H} \cdots \mathrm{N}$ hydrogen bonds in compound (V) only. In compound (III), there are two fairly short intermolecular $\mathrm{C}-\mathrm{H} \cdots \mathrm{N}$ contacts involving $\mathrm{C}-\mathrm{H}$ bonds from methyl groups bonded to the quinoline nucleus: not only are such bonds of low acidity, but these methyl groups are likely to be undergoing very rapid rotation about the adjacent $\mathrm{C}-\mathrm{C}$ bonds 


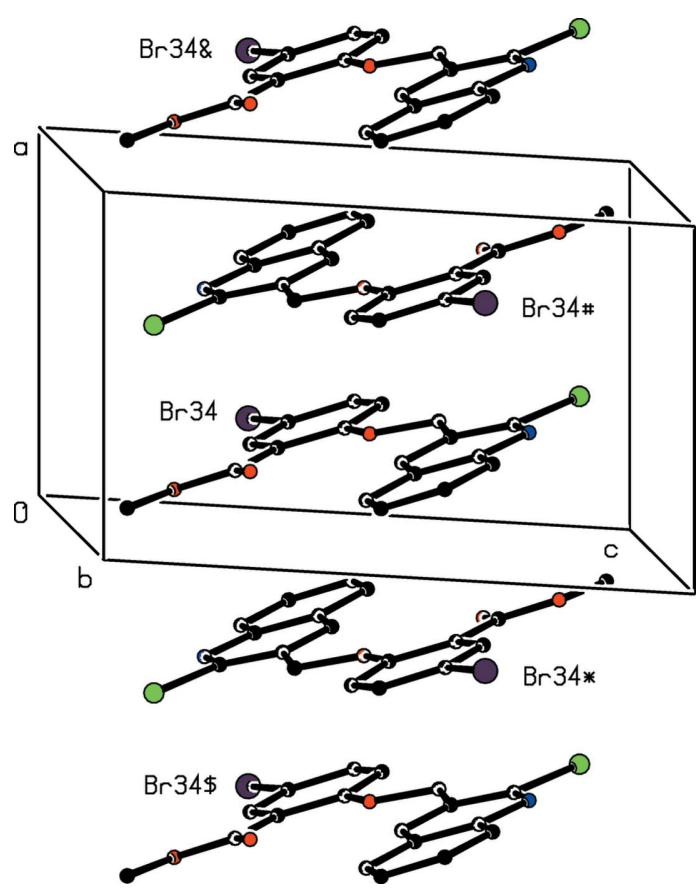

Figure 9

Part of the crystal structure of compound (I) showing the formation of a $\pi$-stacked chain along [100]. For the sake of clarity, H atoms have been omitted. Atoms marked with an asterisk (*), a hash (\#), a dollar sign (\$) or an ampersand $(\&)$ are at the symmetry positions $(-x,-y+1,-z+1)$, $(-x+1,-y+1,-z+1),(x-1, y, z)$ and $(x+1, y, z)$, respectively.

(Riddell \& Rogerson, 1996, 1997). When a group having local $C_{3}$ symmetry, such as a methyl group, is directly bonded to a group having approximate local $C_{2}$ symmetry, such as an aryl ring, the rotational barrier between these two groups is extremely low, of the order of $\mathrm{J} \mathrm{mol}^{-1}$ rather than the usual $\mathrm{kJ}$ $\mathrm{mol}^{-1}$ (Naylor \& Wilson, 1957; Tannenbaum et al., 1956). Accordingly, these contacts in (III) are not regarded as having any structural significance. Likewise, the $\mathrm{C}-\mathrm{H} \cdots \mathrm{O}$ contact in (V) involving the methyl group bonded to the unfused pyridine ring is not regarded as significant.

There are no hydrogen bonds of any kind in the crystal structure of compound (I), but molecules are linked into chains by $\pi-\pi$ stacking interactions. The fused aryl ring of the molecule at $(x, y, z)$ and the brominated ring of the molecule at $(-x+1,-y+1,-z+1)$ make a dihedral angle of $1.04^{\circ}$; the ring centroid separation is $3.6168(10) \AA$, and the shortest perpendicular distance from the centroid of one ring to the plane of the other is 3.4132 (6) $\AA$, with a ring-centroid offset of ca $1.20 \AA$. For the heterocyclic ring at $(x, y, z)$ and the brominated aryl ring at $(-x, 1-y, 1-z)$, the corresponding values are $1.52(9)^{\circ}, 3.7454$ (11) $\AA$, 3.4357 (8) $\AA$ and $c a 1.49 \AA$. The combination of these two stacking interactions links the molecules of (I) into a chain running parallel to the [100] direction (Fig. 9). Two chains of this type pass through each unit cell but there are no direction-specific interactions between adjacent chains.

The only short $\mathrm{C}-\mathrm{H} \cdots \mathrm{O}$ contact in the structure of compound (II) has a $\mathrm{C}-\mathrm{H} \cdots \mathrm{O}$ angle of only $136^{\circ}$ (Table 2), and so it is unlikely to be of major structural significance

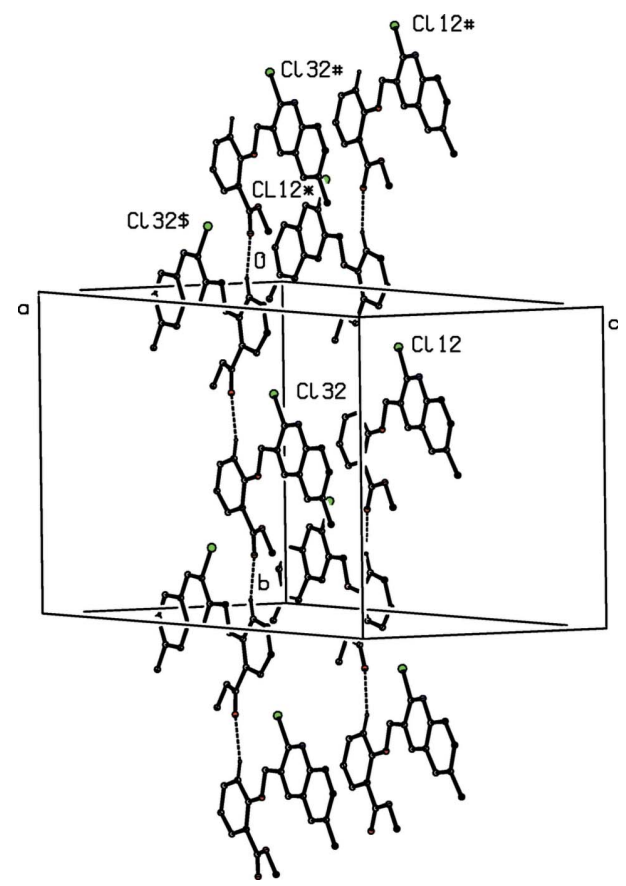

Figure 10

Part of the crystal structure of compound (III) showing the formation of two independent chains running parallel to the [010] direction and formed separately by the molecules of types 1 and 3 . For the sake of clarity, $\mathrm{H}$ atoms not involved in the motifs shown have been omitted. Atoms marked with an asterisk (*), a hash (\#) or a dollar sign (\$) are at the symmetry positions $\left(-x, y-\frac{1}{2},-z+\frac{1}{2}\right),(x, y-1, z)$ and $\left(-x+1, y-\frac{1}{2}\right.$, $\left.-z++\frac{1}{2}\right)$, respectively.

(Wood et al., 2009). However, there is a weak $\pi-\pi$ stacking interaction between molecules related by a $2_{1}$ screw axis. The pyridyl ring at $(x, y, z)$ and the brominated aryl ring at $\left(-x+\frac{1}{2}\right.$, $\left.y+\frac{1}{2}, z\right)$ make a dihedral angle of $3.87(10)^{\circ}$ : the shortest perpendicular distance from the centroid of one ring to the plane of the other is 3.3816 (9) $\AA$, but the ring-centroid separation is 3.882 (12), resulting in a ring-centroid offset of $c a$ $1.78 \AA$. Thus there is only a very modest overlap of these rings and a consequently weak stacking interaction: if this interaction is, in fact, regarded as significant, it links the molecules into a $\pi$-stacked chain running parallel to [010].

Within the selected asymmetric unit for compound (III), three of the four independent molecules, those of types 2, 3 and 4 ( $c f$. Figs. $3-6$ ), are linked by two $\pi-\pi$ stacking interactions, but the type 1 molecule does not participate in any such interaction. One of these stacking interactions involves the pyridyl ring of the type 2 molecule and the fused aryl ring of the type 3 molecule, while the other involves the pyridyl ring of the type 3 molecule and the fused aryl ring of the type 4 molecule. The dihedral angles between the ring planes within these two interactions are 3.11 (18) and $0.96(7)^{\circ}$, respectively, the ring-centroid separations are 3.553 (2) $\AA$ and 3.544 (2) $\AA$, and the shortest perpendicular distances from the centroid of one ring in each interaction to the plane of the other ring are 3.4014 (15) and 3.3820 (15) $\AA$, corresponding to ring-centroid offsets of $c a 1.03$ and $c a 1.06 \AA$, respectively. The only short $\mathrm{C}-\mathrm{H} \cdots \mathrm{N}$ contact within the crystal structure of compound 


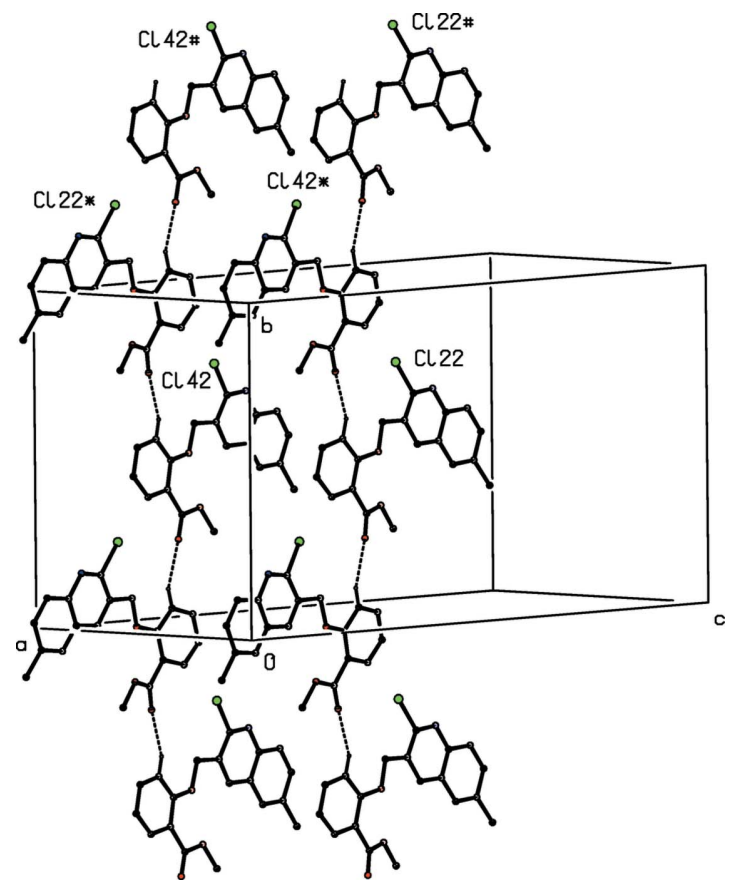

Figure 11

Part of the crystal structure of compound (III) showing the formation of a chain running parallel to the [010] direction and containing alternating molecules of types 2 and 4 . For the sake of clarity, $\mathrm{H}$ atoms not involved in the motifs shown have been omitted. Atoms marked with an asterisk $(*)$ or a hash (\#) are at the symmetry positions $\left(-x+1, y+\frac{1}{2},-z+\frac{1}{2}\right)$ and $(x$, $y+1, z)$, respectively.

(III) has an $\mathrm{H} \cdots \mathrm{N}$ distance which is not significantly less than the sum of the van der Waals radii, but there are four independent $\mathrm{C}-\mathrm{H} \cdots \mathrm{O}$ hydrogen bonds present in the structure although all are probably weak as they have quite small $\mathrm{C}-$ $\mathrm{H} \cdots \mathrm{O}$ angles (Table 2). However, the pattern of these contacts is of interest as it precludes the possibility of any additional crystallographic symmetry in this structure where $Z^{\prime}=4$. One of the $\mathrm{C}-\mathrm{H} \cdots \mathrm{O}$ interactions involves only mol-
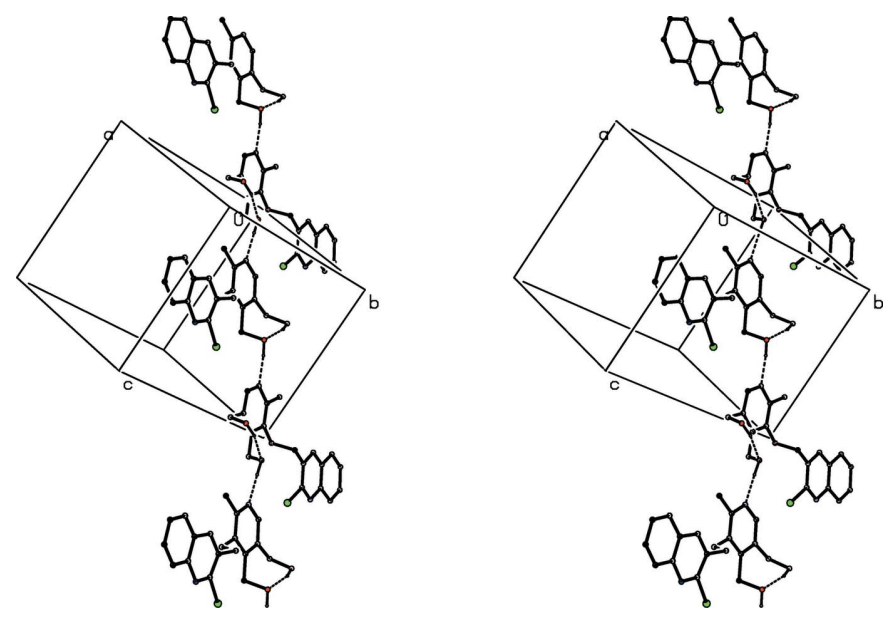

Figure 12

A stereoview of part of the crystal structure of compound $(\mathrm{V})$ showing the formation of a $C(7)$ chain formed by $\mathrm{O}-\mathrm{H} \cdots \mathrm{N}$ hydrogen bonds and running parallel to $[10 \overline{1}]$. For the sake of clarity, $\mathrm{H}$ atoms bonded to $\mathrm{C}$ atoms have been omitted.

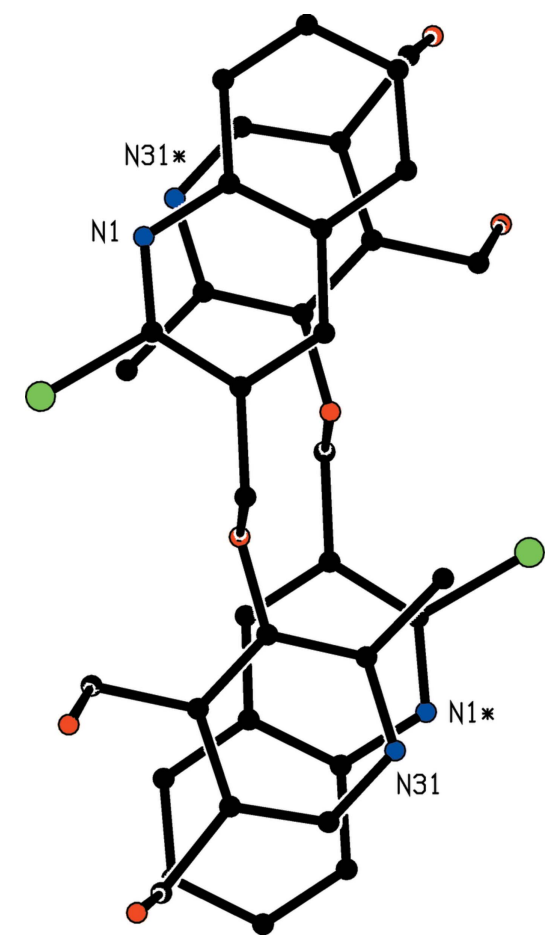

Figure 13

Part of the crystal structure of compound (V) showing the formation of a centrosymmetric $\pi$-stacked dimer. For the sake of clarity, $\mathrm{H}$ atoms have all been omitted. Atoms marked with an asterisk $(*)$ are at the symmetry position $(-x+1,-y+1,-z+1)$.

ecules of type 1 which are related by the $2_{1}$ screw axis along $\left(0, y, \frac{1}{4}\right)$, forming a $C(6)$ (Bernstein et al., 1995) running parallel to the [010] direction (Fig. 10); an entirely similar chain is formed by type 3 molecules related to one another by the $2_{1}$ screw axis along $\left(\frac{1}{2}, y, \frac{1}{4}\right)$. However, the molecules of types 2 and 4 which are related by the $2_{1}$ screw axis along $\left(\frac{1}{2}, y, \frac{1}{4}\right)$ together form a $C_{2}^{2}(12)$ chain parallel to [010] (Fig. 11), which runs antiparallel to the chains formed by the molecules of types 1 and 3 . Hence the patterns of supramolecular assembly in compounds (I)-(III), as well as their crystallization characteristics, show significant differences.

There are no hydrogen bonds of the $\mathrm{C}-\mathrm{H} \cdots \mathrm{N}$ or $\mathrm{C}-$ $\mathrm{H}$.. O types in the crystal structure of compound (IV) and, despite the large number of independent aromatic rings, there are no $\pi-\pi$ stacking interactions. The only direction-specific intermolecular interaction is a weak $\mathrm{C}-\mathrm{H} \cdots \pi$ (arene) contact involving molecules related by translation.

The supramolecular assembly in compound (V) is, however, rather more elaborate, resulting in part from the presence of additional hydrogen-bond donors and acceptors in the unfused pyridine unit. An intramolecular $\mathrm{O}-\mathrm{H} \cdots \mathrm{O}$ hydrogen bond (Table 2) gives rise to an $S(7)$ (Bernstein et al., $1995)$ motif, and an intermolecular $\mathrm{O}-\mathrm{H} \cdots \mathrm{N}$ hydrogen bond links molecules related by the $n$-glide plane at $y=\frac{3}{4}$, forming a $C(7)$ chain running parallel to the $[10 \overline{1}]$ direction (Fig. 12). In addition, inversion-related pairs of molecules are linked by $\pi-$ $\pi$ stacking interactions involving the unfused pyridine ring of one molecule and the quinoline unit of the other (Fig. 13). Thus the unfused pyridine ring of the molecule at $(x, y, z)$ and 

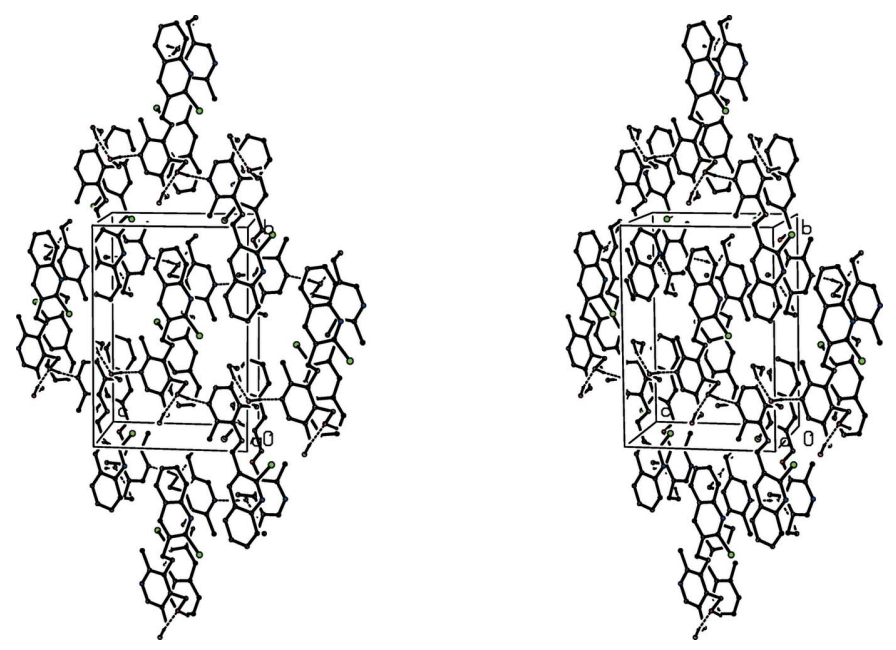

Figure 14

A stereoview of part of the crystal structure of compound (V) showing the formation of a $\pi$-stacked sheet of hydrogen bonded chains lying parallel to (101). For the sake of clarity, $\mathrm{H}$ atoms bonded to $\mathrm{C}$ atoms have been omitted.

the fused pyridine ring of the molecule at $(1-x, 1-y, 1-z)$ make a dihedral angle of $4.43(8)^{\circ}$ : the ring-centroid separation is 3.7499 (9) $\AA$ and the shortest perpendicular distance from the centroid of one ring to the plane of the other is 3.5077 (7) $\AA$, corresponding to a ring-centroid offset of $c a$ $1.33 \AA$ A. For the unfused pyridyl ring at $(x, y, z)$ and the fused aryl ring at $(-x+1,-y+1,-z+1)$ the corresponding values are $1.73(8)^{\circ}, 3.7333(10) \AA, 3.4637$ (8) $\AA$ and $c a 1.39 \AA$, respectively. The effect of the hydrogen-bonded chains is to link the $\pi$-stacked dimer centered at $\left(\frac{1}{2}, \frac{1}{2}, \frac{1}{2}\right)$ directly to the four symmetry-related dimers centred at $(1,0,0),(1,1,0),(0,0,1)$ and $(0,1,1)$, thus forming a sheet of $\pi$-stacked hydrogenbonded chains lying parallel to (101) [Fig. 14].

\section{Database survey}

The structures of a number of fairly simple 2-chloroquinolione derivatives related to compounds (I) $-(\mathrm{V})$ have been reported in recent years. A structural study of a closely related group of six simply substituted 2-chloroquinolines (Hathwar et al., 2010) focused on supramolecular aggregation via $\mathrm{C}-\mathrm{H} \cdots \mathrm{Cl}$ hydrogen bonds and attractive $\mathrm{Cl} \cdots \mathrm{Cl}$ interactions. However, it must be pointed out firstly that it is now well established (Brammer et al., 2001; Thallapally \& Nangia, 2001) that Cl atoms bonded to $\mathrm{C}$ atoms are extremely poor acceptors of hydrogen bonds, even from strong donors such as $\mathrm{O}-\mathrm{H}$ or $\mathrm{N}-\mathrm{H}$; and secondly, that for none of the compounds in this group were the shortest intermolecular $\mathrm{Cl} \cdots \mathrm{Cl}$ distances less than the sum of the van der Waals radii (Bondi, 1964; Nyburg \& Faerman, 1985; Rowland \& Taylor, 1996): indeed, the concept of the van der Waals radius was nowhere mentioned by the original authors. Two of the six compounds in this group contained 3-hydroxymethyl substituents and, in each of these, the molecules are linked into $C(6)$ chains by means of $\mathrm{O}-$ $\mathrm{H} \cdots \mathrm{N}$ hydrogen bonds.
Molecules of 2-[(2-chloroquinolin-3-yl)(hydroxy)methyl]acrylonitrile (Anuradha et al., 2013a) are also linked into $C(6)$ chains by $\mathrm{O}-\mathrm{H} \cdots \mathrm{N}$ hydrogen bonds, while in the closely related methyl 2-[(2-chloroquinolin-3-yl)(hydroxy)methyl]acrylate, where $Z^{\prime}=2$ (Anuradha et al., 2013b), molecules of one type are linked by $\mathrm{O}-\mathrm{H} \cdots \mathrm{O}$ hydrogen bonds, again forming $C(6)$ chains to which the molecules of the second type are linked by $\mathrm{O}-\mathrm{H} \cdots \mathrm{N}$ hydrogen bonds. Chains of $C(6)$ type are formed also in $\mathrm{N}$-\{(2-chloro-3-quinlinyl)methyl]-4-fluoroaniline (Jasinski et al., 2010), which is closely related to compounds (I) $-(\mathrm{V})$ except that an amino linkage replaces the ether linkage in (I)-(V), so that the chains are built from $\mathrm{N}-$ $\mathrm{H} \cdot \cdots \mathrm{N}$ hydrogen bonds.

In the esters (2-choloroquinolin-3-yl)methyl acetate and (2-chloro-6-methylquinolin-3-yl)methyl acetate (Tabassum et al., 2014), there are no strong hydrogen bond donors: in the methylated compound, where $Z^{\prime}=2$, the only hydrogen bond, of $\mathrm{C}-\mathrm{H} \cdots \mathrm{O}$ type, links the two independent molecules, while in the unmethylated compound, the molecules are linked into $C(5)$ chains by $\mathrm{C}-\mathrm{H} \cdots \mathrm{N}$ hydrogen bonds. In the structure of 2-chloro-3-(dimethoxymethyl)-6-methoxyquinoline (Chandrika et al., 2015), there are no hydrogen bonds of any kind.

\section{Synthesis and crystallization}

For the synthesis of compounds (I)-(V), a mixture of $0.4 \mathrm{mmol}$ of the appropriate quinoline derivative, 2-chloro-3-(chloromethyl)quinoline for compounds (I), (IV) and (V) or 2chloro-3-(chloromethyl)-5-methylquinoline for compounds (II) and (III) and $0.4 \mathrm{mmol}$ of the appropriate hydroxy compound, methyl 5-bromo-2-hydroxybenzoate for (I) and (II), methyl 2-hydroxybenzoate for (III), 1-hydroxynaphthalene for (IV), or 3-hydroxy-4,5-bis(hydroxymethyl)-2methylpyridinium chloride for $(\mathrm{V})$, were dissolved in $\mathrm{N}, \mathrm{N}$ dimethylformamide $(3-5 \mathrm{ml})$ together with potassium carbonate $(2 \mathrm{mmol})$ and these mixtures were stirred at ambient temperature for $6-9 \mathrm{~h}$, with monitoring by TLC. When each reaction was complete, ice-cold water $(5 \mathrm{ml})$ was added and the resulting solid products were collected by filtration, washed with water and dried in air. Crystals suitable for singlecrystal X-ray diffraction were obtained by slow evaporation, at ambient temperature and in the presence of air, of solutions in dichloromethane, with yields in the range $86-97 \%$.

\section{Refinement}

Crystal data, data collection and structure refinement details are summarized in Table 3. All $\mathrm{H}$ atoms were located in difference Fourier maps. C-bound $\mathrm{H}$ atoms were then treated as riding atoms in geometrically idealized positions: $\mathrm{C}-\mathrm{H}$ distances $0.95-0.99 \AA$ 遖 with $U_{\text {iso }}(\mathrm{H})=1.5 U_{\text {eq }}(\mathrm{C})$ for the methyl groups, which were permitted to rotate but not to tilt, and $1.2 U_{\text {eq }}(\mathrm{C})$ for other C-bound $\mathrm{H}$ atoms.

The $\mathrm{H}$ atoms bonded to $\mathrm{O}$ atoms in compound $(\mathrm{V})$ were permitted to ride at the positions located in the difference Fourier map, with $U_{\text {iso }}(\mathrm{H})=1.5 U_{\text {eq }}(\mathrm{O})$, giving $\mathrm{O}-\mathrm{H}$ distances of $0.91 \AA$. For compound (III), the Flack $x$ parameter (Flack, 
Table 3

Experimental details.

(I) (II)

Crystal data

Chemical formula

$M_{\mathrm{r}}$

Crystal system, space group

Temperature (K)

$a, b, c(\AA)$

$\alpha, \beta, \gamma\left({ }^{\circ}\right)$

$V\left(\AA^{3}\right)$

$Z$

Radiation type

$\mu\left(\mathrm{mm}^{-1}\right)$

Crystal size (mm)

$\mathrm{C}_{18} \mathrm{H}_{13} \mathrm{BrClNO}_{3}$

406.64

Monoclinic, $P 2_{1} / n$

173

7.3185 (4), 18.4177 (7), $11.7870(5)$

$90,93.609(4), 90$

1585.62 (13)

4

Mo $K \alpha$

2.78

$0.44 \times 0.23 \times 0.12$

Agilent Eos Gemini

Multi-scan ( $S A D A B S$.

Sheldrick, 2003)

Diffractometer

Absorption correction

$T_{\min }, T_{\max }$

No. of measured, independent and observed $[I>2 \sigma(I)]$ reflections

$R_{\text {int }}$

$(\sin \theta / \lambda)_{\max }\left(\AA^{-1}\right)$

$0.335,0.717$

$17735,4612,3682$

0.036

0.703

$0.033,0.071,1.06$

4612

218

No. of reflections

No. of parameters

No. of restraints

$\mathrm{H}$-atom treatment

$\Delta \rho_{\max }, \Delta \rho_{\min }\left(\mathrm{e} \AA^{-3}\right)$

Absolute structure

Absolute structure parameter

$0.42,-0.41$

\section{$\mathrm{C}_{19} \mathrm{H}_{15} \mathrm{BrClNO}_{3}$}

420.67

Orthorhombic, $\mathrm{Pbca}$

173

15.1920 (3), 11.98641 (19), 19.0307 (3)

$90,90,90$

3465.44 (10)

8

$\mathrm{Cu} K \alpha$

4.81

$0.24 \times 0.16 \times 0.08$

\section{$\mathrm{C}_{19} \mathrm{H}_{16} \mathrm{ClNO}_{3}$}

341.78

Orthorhombic, $P 2_{1} 2_{1} 2_{1}$

173

13.5860 (3), 15.5857 (2), 30.9389 (5)

90, 90,90

$6551.2(2)$

16

$\mathrm{Cu} \mathrm{K \alpha}$

2.21

$0.48 \times 0.26 \times 0.14$

Agilent Eos Gemini

Multi-scan (SADABS;

Sheldrick, 2003)

$0.472,0.734$

45901, 12840, 11257

0.048

0.619

$0.046,0.129,1.04$

12840

874

0

$\mathrm{H}$-atom parameters constrained $\quad \mathrm{H}$-atom parameters constrained

$0.52,-0.49$

H-atom parameters constrained

$0.42,-0.31$

Refined as an inversion twin 0.152 (16)

\section{(IV)}

(V)

Crystal data

Chemical formula

$M_{\mathrm{r}}$

Crystal system, space group

Temperature $(\mathrm{K})$

$a, b, c(\AA)$

$\alpha, \beta, \gamma\left({ }^{\circ}\right)$

$V\left(\AA^{3}\right)$

$Z$

Radiation type

$\mu\left(\mathrm{mm}^{-1}\right)$

Crystal size (mm)

Data collection

Diffractometer

Absorption correction

$T_{\min }, T_{\max }$

No. of measured, independent and observed $[I>2 \sigma(I)]$ reflections

$R_{\text {int }}$

$(\sin \theta / \lambda)_{\max }\left(\AA^{-1}\right)$

\section{$\mathrm{C}_{20} \mathrm{H}_{14} \mathrm{ClNO}$}

319.77

Monoclinic, $P 2_{1}$

173

5.3165 (3), 10.5098 (4), 13.6201 (7)

$90,98.527(5), 90$

$752.62(6)$

2

$\mathrm{Cu} K \alpha$

2.27

$0.34 \times 0.10 \times 0.08$

Agilent Eos Gemini

Multi-scan (SADABS; Sheldrick, 2003)

$0.551,0.834$

4606, 2014, 1938

0.029

0.619

$0.033,0.090,1.08$

2014

208

1

$\mathrm{H}$-atom parameters constrained

$0.22,-0.19$

Classical Flack method preferred

over Parsons because s.u. lower -0.007 (18)
$\mathrm{C}_{18} \mathrm{H}_{17} \mathrm{ClN}_{2} \mathrm{O}_{3}$

344.79

Monoclinic, $P 2_{1} / n$

173

9.7866 (3), 15.3336 (4), 10.6570 (3)

90, $92.381(3), 90$

$1597.85(8)$

4

$\mathrm{Cu} K \alpha$

2.29

$0.42 \times 0.38 \times 0.32$

Agilent Eos Gemini

Multi-scan (SADABS; Sheldrick, 2003)

$0.375,0.481$

$9423,3112,2764$

0.043

0.618

$0.045,0.127,1.05$

3112

219

0

$\mathrm{H}$-atom parameters constrained

$0.32,-0.25$

$\Delta \rho_{\max }, \Delta \rho_{\min }\left(\mathrm{e} \AA^{-3}\right)$

Absolute structure parameter

Computer programs: CrysAlis PRO and CrysAlis RED (Agilent, 2012), SHELXS97 (Sheldrick, 2008), SHELXL2014 (Sheldrick, 2015) and PLATON (Spek, 2009). 
1983) for the crystal selected for data collection was $x=$ 0.161 (1) calculated (Parsons et al., 2013) using 4617 quotients of type $\left[\left(I^{+}\right)-\left(I^{-}\right)\right] /\left[\left(I^{+}\right)+\left(I^{-}\right)\right]$. Use of the TWIN/BASF instructions in SHELXL2014 (Sheldrick, 2015) gave a value for the twin fraction of 0.152 (16). For compound (IV), the absolute configuration of the conformational enantiomer present in the crystal selected for data collection was established by means of the Flack $x$ parameter calculated as $x=-0.007$ (18) by the standard method (Flack, 1983) and as $x=0.06(2)$ calculated using 102 quotients of type $\left[\left(I^{+}\right)-\left(I^{-}\right)\right] /\left[\left(I^{+}\right)+\left(I^{-}\right)\right]$.

\section{Acknowledgements}

HBVS and THS thank the authorities of Jain University for their support and encouragement. HSY thanks the University of Mysore for research facilities. JPJ acknowledges the NSFMRI program (grant No. 1039027) for funds to purchase the $\mathrm{X}$-ray diffractometer.

\section{References}

Abdel-Wahab, B. F., Khidre, R. E., Farahat, A. A. \& El-Ahl, A. S. (2012). Arkivoc, (i), 211-276.

Agilent (2012). CrysAlis PRO and CrysAlis RED. Agilent Technologies Ltd, Yarnton, Oxfordshire, England.

Anuradha, T., Srinivasan, J., Seshadri, P. R. \& Bakthadoss, M. (2013a). Acta Cryst. E69, o779.

Anuradha, T., Srinivasan, J., Seshadri, P. R. \& Bakthadoss, M. (2013b). Acta Cryst. E69, o990.

Bernstein, J., Davis, R. E., Shimoni, L. \& Chang, N.-L. (1995). Angew. Chem. Int. Ed. Engl. 34, 1555-1573.

Bondi, A. (1964). J. Phys. Chem. 68, 441-451.
Brammer, L., Bruton, E. A. \& Sherwood, P. (2001). Cryst. Growth Des. 1, 277-290.

Chandrika, N., Suresha Kumara, T. H., Jasinski, J. P., Millikan, S. P., Yathirajan, H. S. \& Glidewell, C. (2015). Acta Cryst. E71, o364o365.

Flack, H. D. (1983). Acta Cryst. A39, 876-881.

Hathwar, V. R., Roopan, S. M., Subashini, R., Khan, F. N. \& Guru Row, T. N. (2010). J. Chem. Soc. (Bangalore), 122, 677-685.

Jasinski, J. P., Pek, A. E., Chidan Kumar, C. S., Yathirajan, H. S. \& Kumar, S. (2010). Acta Cryst. E66, o2548-o2549.

Marella, A., Tanwar, O. P., Saha, R., Ali, M. R., Srivastava, S., Akhter, M., Shaquiquzzaman, M. \& Alam, M. M. (2013). Saudi Pharm. J. 21, 1-12.

Naylor, R. E. \& Wilson, E. B. (1957). J. Chem. Phys. 26, 1057-1060. Nyburg, S. C. \& Faerman, C. H. (1985). Acta Cryst. B41, 274-279.

Parsons, S., Flack, H. D. \& Wagner, T. (2013). Acta Cryst. B69, 249 259.

Riddell, F. G. \& Rogerson, M. (1996). J. Chem. Soc. Perkin Trans. 2, pp. 493-504.

Riddell, F. G. \& Rogerson, M. (1997). J. Chem. Soc. Perkin Trans. 2, pp. 249-256.

Rowland, R. S. \& Taylor, R. (1996). J. Phys. Chem. 100, 7384-7391.

Sheldrick, G. M. (2003). SADABS. University of Göttingen, Germany.

Sheldrick, G. M. (2008). Acta Cryst. A64, 112-122.

Sheldrick, G. M. (2015). Acta Cryst. C71, 3-8.

Spek, A. L. (2009). Acta Cryst. D65, 148-155.

Tabassum, S., Suresha Kumara, T. H., Jasinski, J. P., Millikan, S. P., Yathirajan, H. S., Sujan Ganapathy, P. S., Sowmya, H. V., More, S. S., Nagendrappa, G., Kaur, M. \& Jose, G. (2014). J. Mol. Struct. 1070, 10-20.

Tannenbaum, E., Myers, R. J. \& Gwinn, W. D. (1956). J. Chem. Phys. 25, 42-47.

Thallapally, P. K. \& Nangia, A. (2001). CrystEngComm, 3, 114-119.

Wood, P. A., Allen, F. H. \& Pidcock, E. (2009). CrystEngComm, 11, 1563-1571. 


\section{supporting information}

Acta Cryst. (2015). E71, 609-617 [doi:10.1107/S2056989015008233]

\section{Crystal structures of five (2-chloroquinolin-3-yl)methyl ethers: supramolecular assembly in one and two dimensions mediated by hydrogen bonding and $\pi-\pi$ stacking}

Haliwana B. V. Sowmya, Tholappanavara H. Suresha Kumara, Nagendrappa Gopalpur, Jerry P. Jasinski, Sean P. Millikan, Hemmige S. Yathirajan and Christopher Glidewell

Computing details

For all compounds, data collection: CrysAlis PRO (Agilent, 2012); cell refinement: CrysAlis PRO (Agilent, 2012); data reduction: CrysAlis RED (Agilent, 2012); program(s) used to solve structure: SHELXS97 (Sheldrick, 2008); program(s) used to refine structure: SHELXL2014 (Sheldrick, 2015); molecular graphics: PLATON (Spek, 2009); software used to prepare material for publication: SHELXL2014 (Sheldrick, 2015) and PLATON (Spek, 2009).

(I) Methyl 5-bromo-2-[(2-chloroquinolin-3-yl)methoxy]benzoate

Crystal data

$\mathrm{C}_{18} \mathrm{H}_{13} \mathrm{BrClNO}_{3}$

$M_{r}=406.64$

Monoclinic, $P 2_{1} / n$

$a=7.3185(4) \AA$

$b=18.4177(7) \AA$

$c=11.7870(5) \AA$

$\beta=93.609(4)^{\circ}$

$V=1585.62(13) \AA^{3}$

$Z=4$

Data collection

Agilent Eos Gemini diffractometer

Radiation source: Enhance (Mo) X-ray Source $\omega$ scans

Absorption correction: multi-scan

(SADABS; Sheldrick, 2003)

$T_{\min }=0.335, T_{\max }=0.717$

17735 measured reflections

Refinement

Refinement on $F^{2}$

Least-squares matrix: full

$R\left[F^{2}>2 \sigma\left(F^{2}\right)\right]=0.033$

$w R\left(F^{2}\right)=0.071$

$S=1.06$

4612 reflections
$F(000)=816$

$D_{\mathrm{x}}=1.703 \mathrm{Mg} \mathrm{m}^{-3}$

Mo $K \alpha$ radiation, $\lambda=0.71073 \AA$

Cell parameters from 5443 reflections

$\theta=3.0-33.0^{\circ}$

$\mu=2.78 \mathrm{~mm}^{-1}$

$T=173 \mathrm{~K}$

Plate, colourles

$0.44 \times 0.23 \times 0.12 \mathrm{~mm}$

4612 independent reflections

3682 reflections with $I>2 \sigma(I)$

$R_{\text {int }}=0.036$

$\theta_{\text {max }}=30.0^{\circ}, \theta_{\min }=3.0^{\circ}$

$h=-10 \rightarrow 8$

$k=-24 \rightarrow 25$

$l=-16 \rightarrow 16$

218 parameters

0 restraints

Hydrogen site location: inferred from neighbouring sites

$\mathrm{H}$-atom parameters constrained 
$w=1 /\left[\sigma^{2}\left(F_{\mathrm{o}}^{2}\right)+(0.0288 P)^{2}+0.6015 P\right]$

where $P=\left(F_{\mathrm{o}}^{2}+2 F_{\mathrm{c}}^{2}\right) / 3$

$(\Delta / \sigma)_{\max }=0.001$

$$
\Delta \rho_{\max }=0.42 \text { e } \AA^{-3}
$$

$\Delta \rho_{\min }=-0.41 \mathrm{e} \AA^{-3}$

\section{Special details}

Geometry. All e.s.d.'s (except the e.s.d. in the dihedral angle between two 1.s. planes) are estimated using the full covariance matrix. The cell e.s.d.'s are taken into account individually in the estimation of e.s.d.'s in distances, angles and torsion angles; correlations between e.s.d.'s in cell parameters are only used when they are defined by crystal symmetry. An approximate (isotropic) treatment of cell e.s.d.'s is used for estimating e.s.d.'s involving l.s. planes.

Fractional atomic coordinates and isotropic or equivalent isotropic displacement parameters $\left(\hat{A}^{2}\right)$

\begin{tabular}{|c|c|c|c|c|}
\hline & $x$ & $y$ & $z$ & $U_{\text {iso }} * / U_{\text {eq }}$ \\
\hline N1 & $0.3544(2)$ & $0.64638(8)$ & $0.75859(12)$ & $0.0228(3)$ \\
\hline $\mathrm{C} 2$ & $0.3622(2)$ & $0.57729(10)$ & 0.74061 (14) & $0.0218(4)$ \\
\hline $\mathrm{Cl} 2$ & $0.44084(7)$ & $0.52448(3)$ & $0.85755(4)$ & $0.03157(12)$ \\
\hline $\mathrm{C} 3$ & $0.3125(2)$ & $0.54040(10)$ & $0.63773(15)$ & $0.0209(4)$ \\
\hline $\mathrm{C} 4$ & $0.2421(2)$ & $0.58250(10)$ & $0.55035(15)$ & $0.0219(4)$ \\
\hline $\mathrm{H} 4$ & 0.2034 & 0.5606 & 0.4799 & $0.026^{*}$ \\
\hline $\mathrm{C} 4 \mathrm{~A}$ & $0.2265(2)$ & $0.65814(10)$ & $0.56389(15)$ & $0.0209(4)$ \\
\hline $\mathrm{C} 5$ & $0.1545(3)$ & $0.70490(11)$ & $0.47672(16)$ & $0.0257(4)$ \\
\hline H5 & 0.1115 & 0.6851 & 0.4055 & $0.031^{*}$ \\
\hline C6 & $0.1462(3)$ & $0.77791(11)$ & $0.49390(17)$ & $0.0303(4)$ \\
\hline H6 & 0.0952 & 0.8087 & 0.4354 & $0.036^{*}$ \\
\hline $\mathrm{C} 7$ & $0.2134(3)$ & $0.80793(11)$ & $0.59853(18)$ & $0.0315(4)$ \\
\hline $\mathrm{H} 7$ & 0.2101 & 0.8590 & 0.6093 & $0.038^{*}$ \\
\hline $\mathrm{C} 8$ & $0.2826(3)$ & $0.76456(11)$ & $0.68391(17)$ & $0.0276(4)$ \\
\hline $\mathrm{H} 8$ & 0.3271 & 0.7855 & 0.7539 & $0.033^{*}$ \\
\hline C8A & $0.2888(2)$ & $0.68865(10)$ & $0.66944(14)$ & $0.0212(4)$ \\
\hline C37 & $0.3404(3)$ & 0.46017 (10) & $0.62654(15)$ & $0.0246(4)$ \\
\hline H37A & 0.4696 & 0.4471 & 0.6467 & $0.030^{*}$ \\
\hline H37B & 0.2612 & 0.4333 & 0.6772 & $0.030^{*}$ \\
\hline $\mathrm{O} 31$ & $0.2926(2)$ & $0.44326(7)$ & $0.51108(10)$ & $0.0275(3)$ \\
\hline $\mathrm{C} 31$ & $0.2926(2)$ & $0.37331(9)$ & $0.47662(14)$ & $0.0203(3)$ \\
\hline $\mathrm{C} 32$ & $0.2264(2)$ & $0.35949(9)$ & $0.36438(14)$ & $0.0191(3)$ \\
\hline $\mathrm{C} 33$ & $0.2199(2)$ & $0.28798(9)$ & $0.32599(14)$ & $0.0203(3)$ \\
\hline H33 & 0.1751 & 0.2779 & 0.2502 & $0.024 *$ \\
\hline C34 & $0.2772(3)$ & $0.23189(9)$ & $0.39624(15)$ & $0.0212(4)$ \\
\hline $\mathrm{Br} 34$ & $0.26437(3)$ & $0.13590(2)$ & $0.33927(2)$ & $0.03066(7)$ \\
\hline $\mathrm{C} 35$ & $0.3456(3)$ & $0.24530(10)$ & $0.50667(15)$ & $0.0235(4)$ \\
\hline H35 & 0.3860 & 0.2064 & 0.5548 & $0.028^{*}$ \\
\hline $\mathrm{C} 36$ & $0.3543(3)$ & $0.31595(10)$ & $0.54578(15)$ & $0.0224(4)$ \\
\hline H36 & 0.4030 & 0.3255 & 0.6209 & $0.027 *$ \\
\hline $\mathrm{C} 38$ & $0.1676(2)$ & $0.41935(10)$ & $0.28586(14)$ & $0.0217(4)$ \\
\hline $\mathrm{O} 38$ & $0.1774(2)$ & $0.48297(8)$ & $0.30438(12)$ & $0.0415(4)$ \\
\hline O39 & $0.1018(2)$ & $0.39288(7)$ & 0.18579 (11) & $0.0361(4)$ \\
\hline C39 & $0.0528(3)$ & $0.44627(11)$ & $0.10042(18)$ & $0.0380(5)$ \\
\hline H39A & -0.0067 & 0.4223 & 0.0336 & $0.057^{*}$ \\
\hline Н39B & 0.1633 & 0.4714 & 0.0786 & $0.057 *$ \\
\hline
\end{tabular}


supporting information

\begin{tabular}{|c|c|c|c|c|c|c|}
\hline & $U^{11}$ & $U^{22}$ & $U^{33}$ & $U^{12}$ & $U^{13}$ & $U^{23}$ \\
\hline N1 & $0.0241(8)$ & $0.0260(8)$ & $0.0181(7)$ & $-0.0004(6)$ & $0.0007(6)$ & $-0.0038(6)$ \\
\hline $\mathrm{C} 2$ & $0.0222(9)$ & $0.0266(9)$ & $0.0164(8)$ & $0.0002(7)$ & $-0.0006(7)$ & $0.0005(7)$ \\
\hline $\mathrm{Cl} 2$ & $0.0414(3)$ & $0.0328(3)$ & $0.0196(2)$ & $0.0036(2)$ & -0.00517 (19) & $0.00316(18)$ \\
\hline $\mathrm{C} 3$ & $0.0220(9)$ & $0.0205(9)$ & $0.0202(8)$ & $-0.0018(7)$ & $0.0021(7)$ & $-0.0023(7)$ \\
\hline $\mathrm{C} 4$ & $0.0240(9)$ & $0.0237(9)$ & $0.0178(8)$ & $-0.0012(7)$ & $0.0002(7)$ & $-0.0028(7)$ \\
\hline $\mathrm{C} 4 \mathrm{~A}$ & $0.0207(9)$ & $0.0222(9)$ & $0.0201(8)$ & $0.0005(7)$ & $0.0031(7)$ & $-0.0006(7)$ \\
\hline $\mathrm{C} 5$ & $0.0272(10)$ & $0.0282(10)$ & $0.0215(8)$ & $0.0036(8)$ & $0.0006(7)$ & $0.0006(7)$ \\
\hline C6 & $0.0314(11)$ & $0.0278(10)$ & $0.0320(10)$ & $0.0073(8)$ & $0.0048(8)$ & $0.0068(8)$ \\
\hline $\mathrm{C} 7$ & $0.0334(11)$ & $0.0228(10)$ & 0.0390 & $0.0045(8)$ & $0.0077(9)$ & $-0.0024(8)$ \\
\hline $\mathrm{C} 8$ & $0.0304(11)$ & $0.0242(10)$ & $0.0282(9)$ & $0.0009(8)$ & $0.0034(8)$ & $-0.0079(8)$ \\
\hline $\mathrm{C} 8 \mathrm{~A}$ & $0.0199(9)$ & $0.0227(9)$ & $0.0213(8)$ & $0.0014(7)$ & $0.0036(7)$ & $-0.0029(7)$ \\
\hline $\mathrm{C} 37$ & $0.0354(11)$ & $0.0211(9)$ & $0.0170(8)$ & $0.0003(7)$ & $-0.0022(7)$ & $-0.0010(7)$ \\
\hline $\mathrm{O} 31$ & $0.0467(9)$ & $0.0168(6)$ & $0.0181(6)$ & $0.0019(6)$ & $-0.0048(6)$ & $-0.0020(5)$ \\
\hline $\mathrm{C} 31$ & $0.0231(9)$ & $0.0188(9)$ & $0.0191(8)$ & $-0.0002(7)$ & $0.0020(7)$ & $0.0000(6)$ \\
\hline $\mathrm{C} 32$ & $0.0215(9)$ & $0.0172(8)$ & $0.0186(8)$ & $0.0003(6)$ & $0.0009(6)$ & $0.0019(6)$ \\
\hline $\mathrm{C} 33$ & $0.0228(9)$ & $0.0187(8)$ & $0.0193(8)$ & $-0.0016(7)$ & $0.0003(7)$ & $0.0000(6)$ \\
\hline $\mathrm{C} 34$ & $0.0250(9)$ & $0.0158(8)$ & $0.0227(8)$ & $-0.0012(6)$ & $0.0008(7)$ & $0.0006(7)$ \\
\hline Br34 & $0.04557(13)$ & $0.01513(9)$ & $0.03011(11)$ & $-0.00002(8)$ & $-0.00682(8)$ & $-0.00001(7)$ \\
\hline $\mathrm{C} 35$ & $0.0284(10)$ & $0.0198(9)$ & $0.0224(9)$ & $0.0007(7)$ & $0.0010(7)$ & $0.0058(7)$ \\
\hline $\mathrm{C} 36$ & $0.0280(10)$ & $0.0209(9)$ & $0.0178(8)$ & $-0.0014(7)$ & $-0.0013(7)$ & $0.0026(7)$ \\
\hline $\mathrm{C} 38$ & $0.0247(9)$ & $0.0207(9)$ & $0.0197(8)$ & $0.0003(7)$ & $0.0011(7)$ & $0.0010(7)$ \\
\hline $\mathrm{O} 38$ & $0.0816(12)$ & $0.0175(7)$ & $0.0240(7)$ & $0.0027(7)$ & $-0.0074(7)$ & $0.0014(6)$ \\
\hline O39 & $0.0601(10)$ & $0.0178(7)$ & $0.0272(7)$ & $-0.0043(6)$ & $-0.0220(7)$ & $0.0050(6)$ \\
\hline C39 & $0.0540(14)$ & $0.0263(11)$ & $0.0307(10)$ & $-0.0027(9)$ & $-0.0207(10)$ & $0.0108(8)$ \\
\hline
\end{tabular}

Geometric parameters $\left(A,{ }^{o}\right)$

\begin{tabular}{llll}
\hline $\mathrm{N} 1-\mathrm{C} 2$ & $1.292(2)$ & $\mathrm{C} 37-\mathrm{H} 37 \mathrm{~B}$ & 0.9900 \\
$\mathrm{~N} 1-\mathrm{C} 8 \mathrm{~A}$ & $1.370(2)$ & $\mathrm{O} 31-\mathrm{C} 31$ & $1.351(2)$ \\
$\mathrm{C} 2-\mathrm{C} 3$ & $1.417(2)$ & $\mathrm{C} 31-\mathrm{C} 36$ & $1.392(2)$ \\
$\mathrm{C} 2-\mathrm{C} 2$ & $1.7542(18)$ & $\mathrm{C} 31-\mathrm{C} 32$ & $1.403(2)$ \\
$\mathrm{C} 3-\mathrm{C} 4$ & $1.364(2)$ & $\mathrm{C} 32-\mathrm{C} 33$ & $1.393(2)$ \\
$\mathrm{C} 3-\mathrm{C} 37$ & $1.499(2)$ & $\mathrm{C} 32-\mathrm{C} 38$ & $1.486(2)$ \\
$\mathrm{C} 4-\mathrm{C} 4 \mathrm{~A}$ & $1.408(3)$ & $\mathrm{C} 33-\mathrm{C} 34$ & $1.373(2)$ \\
$\mathrm{C} 4-\mathrm{H} 4$ & 0.9500 & $\mathrm{C} 33-\mathrm{H} 33$ & 0.9500 \\
$\mathrm{C} 4 \mathrm{~A}-\mathrm{C} 8 \mathrm{~A}$ & $1.414(2)$ & $\mathrm{C} 34-\mathrm{C} 35$ & $1.387(2)$ \\
$\mathrm{C} 4 \mathrm{~A}-\mathrm{C} 5$ & $1.417(3)$ & $\mathrm{C} 34-\mathrm{B} 34$ & $1.8913(18)$ \\
$\mathrm{C} 5-\mathrm{C} 6$ & $1.362(3)$ & $\mathrm{C} 35-\mathrm{C} 36$ & $1.380(3)$ \\
$\mathrm{C} 5-\mathrm{H} 5$ & 0.9500 & $\mathrm{C} 35-\mathrm{H} 35$ & 0.9500 \\
$\mathrm{C} 6-\mathrm{C} 7$ & $1.411(3)$ & $\mathrm{C} 36-\mathrm{H} 36$ & 0.9500 \\
$\mathrm{C} 6-\mathrm{H} 6$ & 0.9500 & $\mathrm{C} 38-\mathrm{O} 38$ & $1.193(2)$ \\
$\mathrm{C} 7-\mathrm{C} 8$ & $1.358(3)$ & $\mathrm{C} 38-\mathrm{O} 39$ & $1.338(2)$ \\
$\mathrm{C} 7-\mathrm{H} 7$ & 0.9500 & $\mathrm{O} 39-\mathrm{C} 39$ & $1.436(2)$
\end{tabular}




\begin{tabular}{|c|c|c|c|}
\hline $\mathrm{C} 8-\mathrm{C} 8 \mathrm{~A}$ & $1.410(3)$ & $\mathrm{C} 39-\mathrm{H} 39 \mathrm{~A}$ & 0.9800 \\
\hline $\mathrm{C} 8-\mathrm{H} 8$ & 0.9500 & C39-H39B & 0.9800 \\
\hline $\mathrm{C} 37-\mathrm{O} 31$ & $1.418(2)$ & $\mathrm{C} 39-\mathrm{H} 39 \mathrm{C}$ & 0.9800 \\
\hline C37-H37A & 0.9900 & & \\
\hline $\mathrm{C} 2-\mathrm{N} 1-\mathrm{C} 8 \mathrm{~A}$ & $116.80(15)$ & $\mathrm{C} 3-\mathrm{C} 37-\mathrm{H} 37 \mathrm{~B}$ & 110.6 \\
\hline $\mathrm{N} 1-\mathrm{C} 2-\mathrm{C} 3$ & $126.94(16)$ & $\mathrm{H} 37 \mathrm{~A}-\mathrm{C} 37-\mathrm{H} 37 \mathrm{~B}$ & 108.7 \\
\hline $\mathrm{N} 1-\mathrm{C} 2-\mathrm{Cl} 2$ & $115.65(13)$ & $\mathrm{C} 31-\mathrm{O} 31-\mathrm{C} 37$ & $119.56(14)$ \\
\hline $\mathrm{C} 3-\mathrm{C} 2-\mathrm{Cl} 2$ & $117.41(14)$ & $\mathrm{O} 31-\mathrm{C} 31-\mathrm{C} 36$ & $123.63(15)$ \\
\hline $\mathrm{C} 4-\mathrm{C} 3-\mathrm{C} 2$ & $115.97(16)$ & $\mathrm{O} 31-\mathrm{C} 31-\mathrm{C} 32$ & $116.73(15)$ \\
\hline $\mathrm{C} 4-\mathrm{C} 3-\mathrm{C} 37$ & $122.75(16)$ & $\mathrm{C} 36-\mathrm{C} 31-\mathrm{C} 32$ & $119.63(16)$ \\
\hline $\mathrm{C} 2-\mathrm{C} 3-\mathrm{C} 37$ & $121.27(16)$ & $\mathrm{C} 33-\mathrm{C} 32-\mathrm{C} 31$ & $118.73(15)$ \\
\hline $\mathrm{C} 3-\mathrm{C} 4-\mathrm{C} 4 \mathrm{~A}$ & $120.43(16)$ & $\mathrm{C} 33-\mathrm{C} 32-\mathrm{C} 38$ & $119.78(15)$ \\
\hline $\mathrm{C} 3-\mathrm{C} 4-\mathrm{H} 4$ & 119.8 & $\mathrm{C} 31-\mathrm{C} 32-\mathrm{C} 38$ & $121.48(15)$ \\
\hline $\mathrm{C} 4 \mathrm{~A}-\mathrm{C} 4-\mathrm{H} 4$ & 119.8 & $\mathrm{C} 34-\mathrm{C} 33-\mathrm{C} 32$ & $120.84(16)$ \\
\hline $\mathrm{C} 4-\mathrm{C} 4 \mathrm{~A}-\mathrm{C} 8 \mathrm{~A}$ & $117.96(16)$ & $\mathrm{C} 34-\mathrm{C} 33-\mathrm{H} 33$ & 119.6 \\
\hline $\mathrm{C} 4-\mathrm{C} 4 \mathrm{~A}-\mathrm{C} 5$ & $123.28(16)$ & $\mathrm{C} 32-\mathrm{C} 33-\mathrm{H} 33$ & 119.6 \\
\hline $\mathrm{C} 8 \mathrm{~A}-\mathrm{C} 4 \mathrm{~A}-\mathrm{C} 5$ & $118.75(17)$ & $\mathrm{C} 33-\mathrm{C} 34-\mathrm{C} 35$ & $120.69(16)$ \\
\hline $\mathrm{C} 6-\mathrm{C} 5-\mathrm{C} 4 \mathrm{~A}$ & $120.70(18)$ & $\mathrm{C} 33-\mathrm{C} 34-\mathrm{Br} 34$ & $118.86(13)$ \\
\hline $\mathrm{C} 6-\mathrm{C} 5-\mathrm{H} 5$ & 119.7 & $\mathrm{C} 35-\mathrm{C} 34-\mathrm{Br} 34$ & $120.44(13)$ \\
\hline $\mathrm{C} 4 \mathrm{~A}-\mathrm{C} 5-\mathrm{H} 5$ & 119.7 & $\mathrm{C} 36-\mathrm{C} 35-\mathrm{C} 34$ & $119.18(16)$ \\
\hline $\mathrm{C} 5-\mathrm{C} 6-\mathrm{C} 7$ & $120.03(18)$ & $\mathrm{C} 36-\mathrm{C} 35-\mathrm{H} 35$ & 120.4 \\
\hline $\mathrm{C} 5-\mathrm{C} 6-\mathrm{H} 6$ & 120.0 & $\mathrm{C} 34-\mathrm{C} 35-\mathrm{H} 35$ & 120.4 \\
\hline $\mathrm{C} 7-\mathrm{C} 6-\mathrm{H} 6$ & 120.0 & $\mathrm{C} 35-\mathrm{C} 36-\mathrm{C} 31$ & $120.88(16)$ \\
\hline $\mathrm{C} 8-\mathrm{C} 7-\mathrm{C} 6$ & $120.69(19)$ & $\mathrm{C} 35-\mathrm{C} 36-\mathrm{H} 36$ & 119.6 \\
\hline $\mathrm{C} 8-\mathrm{C} 7-\mathrm{H} 7$ & 119.7 & $\mathrm{C} 31-\mathrm{C} 36-\mathrm{H} 36$ & 119.6 \\
\hline $\mathrm{C} 6-\mathrm{C} 7-\mathrm{H} 7$ & 119.7 & $\mathrm{O} 38-\mathrm{C} 38-\mathrm{O} 39$ & $122.23(16)$ \\
\hline $\mathrm{C} 7-\mathrm{C} 8-\mathrm{C} 8 \mathrm{~A}$ & $120.48(18)$ & $\mathrm{O} 38-\mathrm{C} 38-\mathrm{C} 32$ & $127.08(16)$ \\
\hline $\mathrm{C} 7-\mathrm{C} 8-\mathrm{H} 8$ & 119.8 & $\mathrm{O} 39-\mathrm{C} 38-\mathrm{C} 32$ & $110.68(15)$ \\
\hline $\mathrm{C} 8 \mathrm{~A}-\mathrm{C} 8-\mathrm{H} 8$ & 119.8 & $\mathrm{C} 38-\mathrm{O} 39-\mathrm{C} 39$ & $115.37(15)$ \\
\hline $\mathrm{N} 1-\mathrm{C} 8 \mathrm{~A}-\mathrm{C} 8$ & $118.90(16)$ & $\mathrm{O} 39-\mathrm{C} 39-\mathrm{H} 39 \mathrm{~A}$ & 109.5 \\
\hline $\mathrm{N} 1-\mathrm{C} 8 \mathrm{~A}-\mathrm{C} 4 \mathrm{~A}$ & $121.80(16)$ & $\mathrm{O} 39-\mathrm{C} 39-\mathrm{H} 39 \mathrm{~B}$ & 109.5 \\
\hline $\mathrm{C} 8-\mathrm{C} 8 \mathrm{~A}-\mathrm{C} 4 \mathrm{~A}$ & $119.31(17)$ & $\mathrm{H} 39 \mathrm{~A}-\mathrm{C} 39-\mathrm{H} 39 \mathrm{~B}$ & 109.5 \\
\hline $\mathrm{O} 31-\mathrm{C} 37-\mathrm{C} 3$ & $105.92(14)$ & $\mathrm{O} 39-\mathrm{C} 39-\mathrm{H} 39 \mathrm{C}$ & 109.5 \\
\hline $\mathrm{O} 31-\mathrm{C} 37-\mathrm{H} 37 \mathrm{~A}$ & 110.6 & $\mathrm{H} 39 \mathrm{~A}-\mathrm{C} 39-\mathrm{H} 39 \mathrm{C}$ & 109.5 \\
\hline $\mathrm{C} 3-\mathrm{C} 37-\mathrm{H} 37 \mathrm{~A}$ & 110.6 & $\mathrm{H} 39 \mathrm{~B}-\mathrm{C} 39-\mathrm{H} 39 \mathrm{C}$ & 109.5 \\
\hline $\mathrm{O} 31-\mathrm{C} 37-\mathrm{H} 37 \mathrm{~B}$ & 110.6 & & \\
\hline $\mathrm{C} 8 \mathrm{~A}-\mathrm{N} 1-\mathrm{C} 2-\mathrm{C} 3$ & $1.0(3)$ & $\mathrm{C} 2-\mathrm{C} 3-\mathrm{C} 37-\mathrm{O} 31$ & $-174.63(17)$ \\
\hline $\mathrm{C} 8 \mathrm{~A}-\mathrm{N} 1-\mathrm{C} 2-\mathrm{Cl} 2$ & $-178.33(13)$ & $\mathrm{C} 3-\mathrm{C} 37-\mathrm{O} 31-\mathrm{C} 31$ & $-175.71(16)$ \\
\hline $\mathrm{N} 1-\mathrm{C} 2-\mathrm{C} 3-\mathrm{C} 4$ & $-2.9(3)$ & $\mathrm{C} 37-\mathrm{O} 31-\mathrm{C} 31-\mathrm{C} 36$ & $-6.4(3)$ \\
\hline $\mathrm{Cl} 2-\mathrm{C} 2-\mathrm{C} 3-\mathrm{C} 4$ & $176.39(14)$ & $\mathrm{C} 37-\mathrm{O} 31-\mathrm{C} 31-\mathrm{C} 32$ & $173.73(17)$ \\
\hline $\mathrm{N} 1-\mathrm{C} 2-\mathrm{C} 3-\mathrm{C} 37$ & $175.89(19)$ & $\mathrm{O} 31-\mathrm{C} 31-\mathrm{C} 32-\mathrm{C} 33$ & $-178.50(16)$ \\
\hline $\mathrm{C} 12-\mathrm{C} 2-\mathrm{C} 3-\mathrm{C} 37$ & $-4.8(2)$ & $\mathrm{C} 36-\mathrm{C} 31-\mathrm{C} 32-\mathrm{C} 33$ & $1.6(3)$ \\
\hline $\mathrm{C} 2-\mathrm{C} 3-\mathrm{C} 4-\mathrm{C} 4 \mathrm{~A}$ & $1.6(3)$ & $\mathrm{O} 31-\mathrm{C} 31-\mathrm{C} 32-\mathrm{C} 38$ & $2.8(3)$ \\
\hline $\mathrm{C} 37-\mathrm{C} 3-\mathrm{C} 4-\mathrm{C} 4 \mathrm{~A}$ & $-177.14(17)$ & $\mathrm{C} 36-\mathrm{C} 31-\mathrm{C} 32-\mathrm{C} 38$ & $-177.03(17)$ \\
\hline $\mathrm{C} 3-\mathrm{C} 4-\mathrm{C} 4 \mathrm{~A}-\mathrm{C} 8 \mathrm{~A}$ & $1.1(3)$ & $\mathrm{C} 31-\mathrm{C} 32-\mathrm{C} 33-\mathrm{C} 34$ & $-0.1(3)$ \\
\hline $\mathrm{C} 3-\mathrm{C} 4-\mathrm{C} 4 \mathrm{~A}-\mathrm{C} 5$ & $-179.93(18)$ & $\mathrm{C} 38-\mathrm{C} 32-\mathrm{C} 33-\mathrm{C} 34$ & $178.58(17)$ \\
\hline
\end{tabular}




$\begin{array}{llll}\mathrm{C} 4-\mathrm{C} 4 \mathrm{~A}-\mathrm{C} 5-\mathrm{C} 6 & -178.59(19) & \mathrm{C} 32-\mathrm{C} 33-\mathrm{C} 34-\mathrm{C} 35 & -1.0(3) \\ \mathrm{C} 8 \mathrm{~A}-\mathrm{C} 4 \mathrm{~A}-\mathrm{C} 5-\mathrm{C} 6 & 0.3(3) & \mathrm{C} 32-\mathrm{C} 33-\mathrm{C} 34-\mathrm{Br} 34 & 179.85(14) \\ \mathrm{C} 4 \mathrm{~A}-\mathrm{C} 5-\mathrm{C} 6-\mathrm{C} 7 & 1.4(3) & \mathrm{C} 33-\mathrm{C} 34-\mathrm{C} 35-\mathrm{C} 36 & 0.5(3) \\ \mathrm{C} 5-\mathrm{C} 6-\mathrm{C} 7-\mathrm{C} 8 & -1.6(3) & \mathrm{B} 34-\mathrm{C} 34-\mathrm{C} 35-\mathrm{C} 36 & 179.65(14) \\ \mathrm{C} 6-\mathrm{C} 7-\mathrm{C} 8-\mathrm{C} 8 \mathrm{~A} & 0.1(3) & \mathrm{C} 34-\mathrm{C} 35-\mathrm{C} 36-\mathrm{C} 31 & 1.1(3) \\ \mathrm{C} 2-\mathrm{N} 1-\mathrm{C} 8 \mathrm{~A}-\mathrm{C} 8 & -178.21(17) & \mathrm{O} 31-\mathrm{C} 31-\mathrm{C} 36-\mathrm{C} 35 & 178.00(17) \\ \mathrm{C} 2-\mathrm{N} 1-\mathrm{C} 8 \mathrm{~A}-\mathrm{C} 4 \mathrm{~A} & 2.2(3) & \mathrm{C} 32-\mathrm{C} 31-\mathrm{C} 36-\mathrm{C} 35 & -2.2(3) \\ \mathrm{C} 7-\mathrm{C} 8-\mathrm{C} 8 \mathrm{~A}-\mathrm{N} 1 & -178.03(18) & \mathrm{C} 33-\mathrm{C} 32-\mathrm{C} 38-\mathrm{O} 38 & -174.5(2) \\ \mathrm{C} 7-\mathrm{C} 8-\mathrm{C} 8 \mathrm{~A}-\mathrm{C} 4 \mathrm{~A} & 1.6(3) & \mathrm{C} 31-\mathrm{C} 32-\mathrm{C} 38-\mathrm{O} 38 & 4.1(3) \\ \mathrm{C} 4-\mathrm{C} 4 \mathrm{~A}-\mathrm{C} 8 \mathrm{~A}-\mathrm{N} 1 & -3.2(3) & \mathrm{C} 33-\mathrm{C} 32-\mathrm{C} 38-\mathrm{O} 39 & 4.3(2) \\ \mathrm{C} 5-\mathrm{C} 4 \mathrm{~A}-\mathrm{C} 8 \mathrm{~A}-\mathrm{N} 1 & 177.82(17) & \mathrm{C} 31-\mathrm{C} 32-\mathrm{C} 38-\mathrm{O} 39 & -177.01(17) \\ \mathrm{C} 4-\mathrm{C} 4 \mathrm{~A}-\mathrm{C} 8 \mathrm{~A}-\mathrm{C} 8 & 177.18(17) & \mathrm{O} 38-\mathrm{C} 38-\mathrm{O} 39-\mathrm{C} 39 & 3.1(3) \\ \mathrm{C} 5-\mathrm{C} 4 \mathrm{~A}-\mathrm{C} 8 \mathrm{~A}-\mathrm{C} 8 & -1.8(3) & \mathrm{C} 32-\mathrm{C} 38-\mathrm{O} 39-\mathrm{C} 39 & -175.77(17) \\ \mathrm{C} 4-\mathrm{C} 3-\mathrm{C} 37-\mathrm{O} 31 & 4.1(3) & & \end{array}$

(II) Methyl 5-bromo-2-[(2-chloro-6-methylquinolin-3-yl)methoxy]benzoate

Crystal data

$\mathrm{C}_{19} \mathrm{H}_{15} \mathrm{BrClNO}_{3}$

$M_{r}=420.67$

Orthorhombic, $\mathrm{Pbca}$

$a=15.1920(3) \AA$

$b=11.98641(19) \AA$

$c=19.0307(3) \AA$

$V=3465.44(10) \AA^{3}$

$Z=8$

$F(000)=1696$

\section{Data collection}

Agilent Eos Gemini diffractometer

Radiation source: Enhance $(\mathrm{Cu}) \mathrm{X}$-ray Source $\omega$ scans

Absorption correction: multi-scan

(SADABS; Sheldrick, 2003)

$T_{\min }=0.399, T_{\max }=0.680$

21861 measured reflections

\section{Refinement}

Refinement on $F^{2}$

Least-squares matrix: full

$R\left[F^{2}>2 \sigma\left(F^{2}\right)\right]=0.034$

$w R\left(F^{2}\right)=0.093$

$S=1.06$

3421 reflections

229 parameters

0 restraints

Hydrogen site location: inferred from neighbouring sites
$D_{\mathrm{x}}=1.613 \mathrm{Mg} \mathrm{m}^{-3}$

$\mathrm{Cu} K \alpha$ radiation, $\lambda=1.54184 \AA$

Cell parameters from 3421 reflections

$\theta=4.7-72.5^{\circ}$

$\mu=4.81 \mathrm{~mm}^{-1}$

$T=173 \mathrm{~K}$

Block, colourless

$0.24 \times 0.16 \times 0.08 \mathrm{~mm}$

3421 independent reflections

3062 reflections with $I>2 \sigma(I)$

$R_{\text {int }}=0.055$

$\theta_{\max }=72.5^{\circ}, \theta_{\min }=4.7^{\circ}$

$h=-18 \rightarrow 18$

$k=-14 \rightarrow 12$

$l=-23 \rightarrow 18$

$\mathrm{H}$-atom parameters constrained

$w=1 /\left[\sigma^{2}\left(F_{\mathrm{o}}^{2}\right)+(0.0523 P)^{2}+1.8465 P\right]$

where $P=\left(F_{\mathrm{o}}^{2}+2 F_{\mathrm{c}}^{2}\right) / 3$

$(\Delta / \sigma)_{\max }<0.001$

$\Delta \rho_{\max }=0.52$ e $\AA^{-3}$

$\Delta \rho_{\min }=-0.49$ e $\AA^{-3}$

Extinction correction: SHELXL2014 (Sheldrick, 2015), $\mathrm{Fc}^{*}=\mathrm{kFc}\left[1+0.001 \mathrm{xFc}^{2} \lambda^{3} / \sin (2 \theta)\right]^{-1 / 4}$

Extinction coefficient: 0.00048 (6) 


\section{Special details}

Geometry. All e.s.d.'s (except the e.s.d. in the dihedral angle between two 1.s. planes) are estimated using the full covariance matrix. The cell e.s.d.'s are taken into account individually in the estimation of e.s.d.'s in distances, angles and torsion angles; correlations between e.s.d.'s in cell parameters are only used when they are defined by crystal symmetry. An approximate (isotropic) treatment of cell e.s.d.'s is used for estimating e.s.d.'s involving l.s. planes.

Fractional atomic coordinates and isotropic or equivalent isotropic displacement parameters $\left(\AA^{2}\right)$

\begin{tabular}{|c|c|c|c|c|}
\hline & $x$ & $y$ & $z$ & $U_{\text {iso }} * / U_{\text {eq }}$ \\
\hline $\mathrm{N} 1$ & $0.42983(12)$ & $0.87307(15)$ & $0.45790(10)$ & $0.0272(4)$ \\
\hline $\mathrm{C} 2$ & $0.42690(13)$ & $0.78272(18)$ & $0.42079(11)$ & $0.0249(4)$ \\
\hline $\mathrm{Cl} 2$ & $0.52936(3)$ & $0.72811(5)$ & $0.39507(3)$ & $0.03655(16)$ \\
\hline $\mathrm{C} 3$ & $0.35033(14)$ & $0.72343(17)$ & $0.40030(10)$ & $0.0226(4)$ \\
\hline $\mathrm{C} 4$ & $0.27189(13)$ & $0.76626(18)$ & $0.42260(11)$ & $0.0224(4)$ \\
\hline $\mathrm{H} 4$ & 0.2185 & 0.7297 & 0.4106 & $0.027^{*}$ \\
\hline $\mathrm{C} 4 \mathrm{~A}$ & $0.26987(13)$ & $0.86525(18)$ & $0.46363(11)$ & $0.0219(4)$ \\
\hline $\mathrm{C} 5$ & $0.19142(14)$ & $0.91446(18)$ & $0.48888(11)$ & $0.0253(4)$ \\
\hline H5 & 0.1366 & 0.8805 & 0.4780 & $0.030^{*}$ \\
\hline C6 & $0.19242(15)$ & $1.00976(18)$ & $0.52857(11)$ & $0.0267(4)$ \\
\hline $\mathrm{C} 7$ & $0.27500(15)$ & $1.05991(19)$ & $0.54421(12)$ & $0.0302(5)$ \\
\hline $\mathrm{H} 7$ & 0.2765 & 1.1261 & 0.5716 & $0.036^{*}$ \\
\hline $\mathrm{C} 8$ & $0.35220(15)$ & 1.01485 (19) & $0.52062(12)$ & $0.0299(5)$ \\
\hline $\mathrm{H} 8$ & 0.4066 & 1.0499 & 0.5316 & $0.036^{*}$ \\
\hline $\mathrm{C} 8 \mathrm{~A}$ & $0.35123(14)$ & $0.91644(17)$ & $0.48007(11)$ & $0.0242(4)$ \\
\hline C37 & 0.35875 & $0.61865(18)$ & $0.35765(11)$ & $0.0243(4)$ \\
\hline H37A & 0.3916 & 0.6338 & 0.3137 & $0.029^{*}$ \\
\hline H37B & 0.3909 & 0.5610 & 0.3846 & $0.029^{*}$ \\
\hline $\mathrm{O} 31$ & $0.27182(9)$ & $0.58119(13)$ & $0.34187(8)$ & $0.0254(3)$ \\
\hline $\mathrm{C} 31$ & $0.26370(13)$ & $0.48636(18)$ & $0.30374(11)$ & $0.0224(4)$ \\
\hline $\mathrm{C} 32$ & $0.17885(14)$ & $0.45623(18)$ & $0.28094(11)$ & $0.0236(4)$ \\
\hline $\mathrm{C} 33$ & $0.16860(15)$ & $0.36086(18)$ & $0.23979(11)$ & $0.0267(4)$ \\
\hline H33 & 0.1116 & 0.3399 & 0.2240 & $0.032 *$ \\
\hline C34 & $0.24049(16)$ & $0.29669(19)$ & $0.22183(11)$ & $0.0271(5)$ \\
\hline $\operatorname{Br} 34$ & $0.22547(2)$ & $0.17010(2)$ & $0.16308(2)$ & $0.03596(12)$ \\
\hline $\mathrm{C} 35$ & $0.32387(16)$ & 0.32597 (18) & $0.24421(12)$ & $0.0297(5)$ \\
\hline H35 & 0.3731 & 0.2813 & 0.2317 & $0.036^{*}$ \\
\hline $\mathrm{C} 36$ & $0.33537(14)$ & $0.42003(18)$ & $0.28463(11)$ & $0.0273(5)$ \\
\hline H36 & 0.3928 & 0.4401 & 0.2997 & $0.033^{*}$ \\
\hline C38 & $0.09631(14)$ & $0.5209(2)$ & $0.29458(12)$ & $0.0309(5)$ \\
\hline $\mathrm{O} 38$ & $0.03146(13)$ & $0.5097(2)$ & $0.25963(13)$ & $0.0739(8)$ \\
\hline O39 & $0.10112(11)$ & $0.59054(16)$ & $0.34798(9)$ & $0.0380(4)$ \\
\hline C39 & $0.02126(19)$ & $0.6494(3)$ & $0.36541(18)$ & $0.0522(7)$ \\
\hline H39A & 0.0089 & 0.7052 & 0.3291 & $0.078^{*}$ \\
\hline H39B & 0.0283 & 0.6866 & 0.4109 & $0.078^{*}$ \\
\hline H39C & -0.0277 & 0.5963 & 0.3680 & $0.078^{*}$ \\
\hline C61 & $0.10926(16)$ & $1.0618(2)$ & $0.55704(13)$ & $0.0365(5)$ \\
\hline H61A & 0.0590 & 1.0382 & 0.5284 & $0.055^{*}$ \\
\hline H61B & 0.1145 & 1.1432 & 0.5555 & $0.055^{*}$ \\
\hline
\end{tabular}


supporting information

H61C

0.1003

1.0376

0.6057

$0.055^{*}$

Atomic displacement parameters $\left(\AA^{2}\right)$

\begin{tabular}{lllllll}
\hline & $U^{11}$ & $U^{22}$ & $U^{33}$ & $U^{12}$ & $U^{13}$ & $U^{23}$ \\
\hline N1 & $0.0241(9)$ & $0.0257(9)$ & $0.0319(10)$ & $-0.0054(7)$ & $-0.0027(7)$ & $-0.0008(8)$ \\
C2 & $0.0193(10)$ & $0.0264(11)$ & $0.0291(11)$ & $-0.0017(8)$ & $0.0002(8)$ & $0.0026(8)$ \\
C12 & $0.0207(3)$ & $0.0373(3)$ & $0.0516(4)$ & $-0.0023(2)$ & $0.0022(2)$ & $-0.0088(3)$ \\
C3 & $0.0236(10)$ & $0.0229(10)$ & $0.0214(10)$ & $-0.0037(8)$ & $-0.0022(8)$ & $0.0021(8)$ \\
C4 & $0.0202(10)$ & $0.0249(10)$ & $0.0222(10)$ & $-0.0041(8)$ & $-0.0023(7)$ & $0.0023(8)$ \\
C4A & $0.0236(10)$ & $0.0226(10)$ & $0.0196(10)$ & $-0.0023(8)$ & $-0.0023(7)$ & $0.0033(8)$ \\
C5 & $0.0248(10)$ & $0.0288(11)$ & $0.0224(10)$ & $-0.0030(9)$ & $-0.0021(8)$ & $0.0012(8)$ \\
C6 & $0.0309(11)$ & $0.0263(11)$ & $0.0228(10)$ & $0.0025(9)$ & $-0.0017(8)$ & $0.0019(8)$ \\
C7 & $0.0397(13)$ & $0.0231(10)$ & $0.0276(11)$ & $-0.0022(9)$ & $-0.0023(9)$ & $-0.0035(9)$ \\
C8 & $0.0308(11)$ & $0.0263(11)$ & $0.0325(11)$ & $-0.0059(9)$ & $-0.0045(9)$ & $-0.0022(9)$ \\
C8A & $0.0262(10)$ & $0.0227(10)$ & $0.0238(10)$ & $-0.0040(8)$ & $-0.0038(8)$ & $0.0014(8)$ \\
C37 & $0.0199(10)$ & $0.0245(10)$ & $0.0285(11)$ & $-0.0029(8)$ & $-0.0007(8)$ & $-0.0021(9)$ \\
O31 & $0.0198(7)$ & $0.0252(8)$ & $0.0312(8)$ & $-0.0013(6)$ & $-0.0016(5)$ & $-0.0079(6)$ \\
C31 & $0.0241(10)$ & $0.0226(10)$ & $0.0204(10)$ & $-0.0015(8)$ & $0.0002(7)$ & $0.0013(8)$ \\
C32 & $0.0230(10)$ & $0.0237(10)$ & $0.0242(10)$ & $-0.0026(8)$ & $-0.0009(8)$ & $0.0037(8)$ \\
C33 & $0.0283(11)$ & $0.0279(11)$ & $0.0238(10)$ & $-0.0068(9)$ & $-0.0032(8)$ & $0.0032(9)$ \\
C34 & $0.0407(12)$ & $0.0212(10)$ & $0.0195(10)$ & $-0.0054(9)$ & $-0.0007(9)$ & $-0.0012(8)$ \\
Br34 & $0.0551(2)$ & $0.02513(16)$ & $0.02764(17)$ & $-0.00542(10)$ & $-0.00230(10)$ & $-0.00488(9)$ \\
C35 & $0.0322(11)$ & $0.0286(12)$ & $0.0283(11)$ & $0.0027(9)$ & $0.0045(9)$ & $-0.0016(9)$ \\
C36 & $0.0225(10)$ & $0.0300(12)$ & $0.0294(11)$ & $-0.0023(9)$ & $0.0004(8)$ & $-0.0022(9)$ \\
C38 & $0.0225(10)$ & $0.0334(12)$ & $0.0369(12)$ & $-0.0003(9)$ & $-0.0027(9)$ & $-0.0007(10)$ \\
O38 & $0.0309(10)$ & $0.100(2)$ & $0.0905(17)$ & $0.0164(11)$ & $-0.0257(11)$ & $-0.0441(16)$ \\
O39 & $0.0232(8)$ & $0.0460(11)$ & $0.0446(10)$ & $0.0068(7)$ & $0.0006(7)$ & $-0.0115(8)$ \\
C39 & $0.0298(13)$ & $0.0636(19)$ & $0.0632(19)$ & $0.0151(13)$ & $0.0099(13)$ & $-0.0070(16)$ \\
C61 & $0.0372(13)$ & $0.0366(13)$ & $0.0357(13)$ & $0.0042(10)$ & $0.0022(10)$ & $-0.0082(10)$ \\
& & & & & & \\
\hline & & & & & & \\
\hline
\end{tabular}

Geometric parameters $\left(\AA,{ }^{\circ}\right)$

\begin{tabular}{llll}
\hline $\mathrm{N} 1-\mathrm{C} 2$ & $1.294(3)$ & $\mathrm{O} 31-\mathrm{C} 31$ & $1.354(3)$ \\
$\mathrm{N} 1-\mathrm{C} 8 \mathrm{~A}$ & $1.369(3)$ & $\mathrm{C} 31-\mathrm{C} 36$ & $1.396(3)$ \\
$\mathrm{C} 2-\mathrm{C} 3$ & $1.418(3)$ & $\mathrm{C} 31-\mathrm{C} 32$ & $1.407(3)$ \\
$\mathrm{C} 2-\mathrm{C} 2$ & $1.758(2)$ & $\mathrm{C} 32-\mathrm{C} 33$ & $1.394(3)$ \\
$\mathrm{C} 3-\mathrm{C} 4$ & $1.365(3)$ & $\mathrm{C} 32-\mathrm{C} 38$ & $1.497(3)$ \\
$\mathrm{C} 3-\mathrm{C} 37$ & $1.501(3)$ & $\mathrm{C} 33-\mathrm{C} 34$ & $1.379(3)$ \\
$\mathrm{C} 4-\mathrm{C} 4 \mathrm{~A}$ & $1.421(3)$ & $\mathrm{C} 33-\mathrm{H} 33$ & 0.9500 \\
$\mathrm{C} 4-\mathrm{H} 4$ & 0.9500 & $\mathrm{C} 34-\mathrm{C} 35$ & $1.382(3)$ \\
$\mathrm{C} 4 \mathrm{~A}-\mathrm{C} 5$ & $1.414(3)$ & $\mathrm{C} 34-\mathrm{Br} 34$ & $1.899(2)$ \\
$\mathrm{C} 4 \mathrm{~A}-\mathrm{C} 8 \mathrm{~A}$ & $1.415(3)$ & $\mathrm{C} 35-\mathrm{C} 36$ & $1.376(3)$ \\
$\mathrm{C} 5-\mathrm{C} 6$ & $1.370(3)$ & $\mathrm{C} 35-\mathrm{H} 35$ & 0.9500 \\
$\mathrm{C} 5-\mathrm{H} 5$ & 0.9500 & $\mathrm{C} 36-\mathrm{H} 36$ & 0.9500 \\
$\mathrm{C} 6-\mathrm{C} 7$ & $1.423(3)$ & $\mathrm{C} 38-\mathrm{O} 38$ & $1.196(3)$ \\
$\mathrm{C} 6-\mathrm{C} 61$ & $1.509(3)$ & $\mathrm{C} 38-\mathrm{O} 39$ & $1.317(3)$ \\
$\mathrm{C} 7-\mathrm{C} 8$ & $1.367(3)$ & $\mathrm{O} 39-\mathrm{C} 39$ & $1.442(3)$
\end{tabular}




\begin{tabular}{|c|c|c|c|}
\hline $\mathrm{C} 7-\mathrm{H} 7$ & 0.9500 & C39-H39A & 0.9800 \\
\hline $\mathrm{C} 8-\mathrm{C} 8 \mathrm{~A}$ & $1.410(3)$ & C39-H39B & 0.9800 \\
\hline $\mathrm{C} 8-\mathrm{H} 8$ & 0.9500 & $\mathrm{C} 39-\mathrm{H} 39 \mathrm{C}$ & 0.9800 \\
\hline $\mathrm{C} 37-\mathrm{O} 31$ & $1.427(2)$ & C61-H61A & 0.9800 \\
\hline C37-H37A & 0.9900 & C61-H61B & 0.9800 \\
\hline C37-H37B & 0.9900 & $\mathrm{C} 61-\mathrm{H} 61 \mathrm{C}$ & 0.9800 \\
\hline $\mathrm{C} 2-\mathrm{N} 1-\mathrm{C} 8 \mathrm{~A}$ & $117.13(18)$ & $\mathrm{O} 31-\mathrm{C} 31-\mathrm{C} 36$ & $123.12(19)$ \\
\hline $\mathrm{N} 1-\mathrm{C} 2-\mathrm{C} 3$ & $126.73(19)$ & $\mathrm{O} 31-\mathrm{C} 31-\mathrm{C} 32$ & $117.66(18)$ \\
\hline $\mathrm{N} 1-\mathrm{C} 2-\mathrm{Cl} 2$ & $115.67(15)$ & $\mathrm{C} 36-\mathrm{C} 31-\mathrm{C} 32$ & $119.2(2)$ \\
\hline $\mathrm{C} 3-\mathrm{C} 2-\mathrm{Cl} 2$ & $117.59(16)$ & $\mathrm{C} 33-\mathrm{C} 32-\mathrm{C} 31$ & $119.1(2)$ \\
\hline $\mathrm{C} 4-\mathrm{C} 3-\mathrm{C} 2$ & $116.24(19)$ & $\mathrm{C} 33-\mathrm{C} 32-\mathrm{C} 38$ & $115.38(19)$ \\
\hline $\mathrm{C} 4-\mathrm{C} 3-\mathrm{C} 37$ & $123.86(18)$ & $\mathrm{C} 31-\mathrm{C} 32-\mathrm{C} 38$ & $125.50(19)$ \\
\hline $\mathrm{C} 2-\mathrm{C} 3-\mathrm{C} 37$ & $119.89(19)$ & $\mathrm{C} 34-\mathrm{C} 33-\mathrm{C} 32$ & $120.5(2)$ \\
\hline $\mathrm{C} 3-\mathrm{C} 4-\mathrm{C} 4 \mathrm{~A}$ & $120.25(18)$ & $\mathrm{C} 34-\mathrm{C} 33-\mathrm{H} 33$ & 119.7 \\
\hline $\mathrm{C} 3-\mathrm{C} 4-\mathrm{H} 4$ & 119.9 & $\mathrm{C} 32-\mathrm{C} 33-\mathrm{H} 33$ & 119.7 \\
\hline $\mathrm{C} 4 \mathrm{~A}-\mathrm{C} 4-\mathrm{H} 4$ & 119.9 & $\mathrm{C} 33-\mathrm{C} 34-\mathrm{C} 35$ & $120.5(2)$ \\
\hline $\mathrm{C} 5-\mathrm{C} 4 \mathrm{~A}-\mathrm{C} 8 \mathrm{~A}$ & $118.7(2)$ & $\mathrm{C} 33-\mathrm{C} 34-\mathrm{Br} 34$ & $119.77(17)$ \\
\hline $\mathrm{C} 5-\mathrm{C} 4 \mathrm{~A}-\mathrm{C} 4$ & $123.60(18)$ & $\mathrm{C} 35-\mathrm{C} 34-\mathrm{Br} 34$ & $119.65(18)$ \\
\hline $\mathrm{C} 8 \mathrm{~A}-\mathrm{C} 4 \mathrm{~A}-\mathrm{C} 4$ & $117.70(18)$ & $\mathrm{C} 36-\mathrm{C} 35-\mathrm{C} 34$ & $119.8(2)$ \\
\hline $\mathrm{C} 6-\mathrm{C} 5-\mathrm{C} 4 \mathrm{~A}$ & $121.7(2)$ & $\mathrm{C} 36-\mathrm{C} 35-\mathrm{H} 35$ & 120.1 \\
\hline $\mathrm{C} 6-\mathrm{C} 5-\mathrm{H} 5$ & 119.1 & $\mathrm{C} 34-\mathrm{C} 35-\mathrm{H} 35$ & 120.1 \\
\hline $\mathrm{C} 4 \mathrm{~A}-\mathrm{C} 5-\mathrm{H} 5$ & 119.1 & $\mathrm{C} 35-\mathrm{C} 36-\mathrm{C} 31$ & $120.9(2)$ \\
\hline $\mathrm{C} 5-\mathrm{C} 6-\mathrm{C} 7$ & $118.5(2)$ & $\mathrm{C} 35-\mathrm{C} 36-\mathrm{H} 36$ & 119.6 \\
\hline $\mathrm{C} 5-\mathrm{C} 6-\mathrm{C} 61$ & $122.2(2)$ & $\mathrm{C} 31-\mathrm{C} 36-\mathrm{H} 36$ & 119.6 \\
\hline $\mathrm{C} 7-\mathrm{C} 6-\mathrm{C} 61$ & $119.2(2)$ & $\mathrm{O} 38-\mathrm{C} 38-\mathrm{O} 39$ & $123.1(2)$ \\
\hline $\mathrm{C} 8-\mathrm{C} 7-\mathrm{C} 6$ & $121.4(2)$ & $\mathrm{O} 38-\mathrm{C} 38-\mathrm{C} 32$ & $122.3(2)$ \\
\hline $\mathrm{C} 8-\mathrm{C} 7-\mathrm{H} 7$ & 119.3 & $\mathrm{O} 39-\mathrm{C} 38-\mathrm{C} 32$ & $114.56(18)$ \\
\hline $\mathrm{C} 6-\mathrm{C} 7-\mathrm{H} 7$ & 119.3 & $\mathrm{C} 38-\mathrm{O} 39-\mathrm{C} 39$ & $116.1(2)$ \\
\hline $\mathrm{C} 7-\mathrm{C} 8-\mathrm{C} 8 \mathrm{~A}$ & $120.1(2)$ & O39- $339-\mathrm{H} 39 \mathrm{~A}$ & 109.5 \\
\hline $\mathrm{C} 7-\mathrm{C} 8-\mathrm{H} 8$ & 120.0 & O39- & 109.5 \\
\hline $\mathrm{C} 8 \mathrm{~A}-\mathrm{C} 8-\mathrm{H} 8$ & 120.0 & $\mathrm{H} 39 \mathrm{~A}-\mathrm{C} 39-\mathrm{H} 39 \mathrm{~B}$ & 109.5 \\
\hline $\mathrm{N} 1-\mathrm{C} 8 \mathrm{~A}-\mathrm{C} 8$ & $118.51(19)$ & $\mathrm{O} 39-\mathrm{C} 39-\mathrm{H} 39 \mathrm{C}$ & 109.5 \\
\hline $\mathrm{N} 1-\mathrm{C} 8 \mathrm{~A}-\mathrm{C} 4 \mathrm{~A}$ & $121.94(19)$ & $\mathrm{H} 39 \mathrm{~A}-\mathrm{C} 39-\mathrm{H} 39 \mathrm{C}$ & 109.5 \\
\hline $\mathrm{C} 8-\mathrm{C} 8 \mathrm{~A}-\mathrm{C} 4 \mathrm{~A}$ & $119.5(2)$ & $\mathrm{H} 39 \mathrm{~B}-\mathrm{C} 39-\mathrm{H} 39 \mathrm{C}$ & 109.5 \\
\hline $\mathrm{O} 31-\mathrm{C} 37-\mathrm{C} 3$ & $107.35(16)$ & C6- $661-\mathrm{H} 61 \mathrm{~A}$ & 109.5 \\
\hline $\mathrm{O} 31-\mathrm{C} 37-\mathrm{H} 37 \mathrm{~A}$ & 110.2 & $\mathrm{C} 6-\mathrm{C} 61-\mathrm{H} 61 \mathrm{~B}$ & 109.5 \\
\hline $\mathrm{C} 3-\mathrm{C} 37-\mathrm{H} 37 \mathrm{~A}$ & 110.2 & $\mathrm{H} 61 \mathrm{~A}-\mathrm{C} 61-\mathrm{H} 61 \mathrm{~B}$ & 109.5 \\
\hline $\mathrm{O} 31-\mathrm{C} 37-\mathrm{H} 37 \mathrm{~B}$ & 110.2 & $\mathrm{C} 6-\mathrm{C} 61-\mathrm{H} 61 \mathrm{C}$ & 109.5 \\
\hline $\mathrm{C} 3-\mathrm{C} 37-\mathrm{H} 37 \mathrm{~B}$ & 110.2 & $\mathrm{H} 61 \mathrm{~A}-\mathrm{C} 61-\mathrm{H} 61 \mathrm{C}$ & 109.5 \\
\hline $\mathrm{H} 37 \mathrm{~A}-\mathrm{C} 37-\mathrm{H} 37 \mathrm{~B}$ & 108.5 & $\mathrm{H} 61 \mathrm{~B}-\mathrm{C} 61-\mathrm{H} 61 \mathrm{C}$ & 109.5 \\
\hline $\mathrm{C} 31-\mathrm{O} 31-\mathrm{C} 37$ & $117.47(16)$ & & \\
\hline $\mathrm{C} 8 \mathrm{~A}-\mathrm{N} 1-\mathrm{C} 2-\mathrm{C} 3$ & $0.2(3)$ & $\mathrm{C} 4-\mathrm{C} 3-\mathrm{C} 37-\mathrm{O} 31$ & $4.3(3)$ \\
\hline $\mathrm{C} 8 \mathrm{~A}-\mathrm{N} 1-\mathrm{C} 2-\mathrm{Cl} 2$ & $-178.63(15)$ & $\mathrm{C} 2-\mathrm{C} 3-\mathrm{C} 37-\mathrm{O} 31$ & $-176.93(18)$ \\
\hline $\mathrm{N} 1-\mathrm{C} 2-\mathrm{C} 3-\mathrm{C} 4$ & $-0.4(3)$ & $\mathrm{C} 3-\mathrm{C} 37-\mathrm{O} 31-\mathrm{C} 31$ & $-179.57(17)$ \\
\hline $\mathrm{C} 12-\mathrm{C} 2-\mathrm{C} 3-\mathrm{C} 4$ & $178.35(16)$ & $\mathrm{C} 37-\mathrm{O} 31-\mathrm{C} 31-\mathrm{C} 36$ & $5.4(3)$ \\
\hline $\mathrm{N} 1-\mathrm{C} 2-\mathrm{C} 3-\mathrm{C} 37$ & $-179.3(2)$ & $\mathrm{C} 37-\mathrm{O} 31-\mathrm{C} 31-\mathrm{C} 32$ & $-172.62(18)$ \\
\hline
\end{tabular}




$\begin{array}{llll}\mathrm{C} 12-\mathrm{C} 2-\mathrm{C} 3-\mathrm{C} 37 & -0.5(3) & \mathrm{O} 31-\mathrm{C} 31-\mathrm{C} 32-\mathrm{C} 33 & 178.09(18) \\ \mathrm{C} 2-\mathrm{C} 3-\mathrm{C} 4-\mathrm{C} 4 \mathrm{~A} & 0.3(3) & \mathrm{C} 36-\mathrm{C} 31-\mathrm{C} 32-\mathrm{C} 33 & 0.0(3) \\ \mathrm{C} 37-\mathrm{C} 3-\mathrm{C} 4-\mathrm{C} 4 \mathrm{~A} & 179.16(19) & \mathrm{O} 31-\mathrm{C} 31-\mathrm{C} 32-\mathrm{C} 38 & 0.7(3) \\ \mathrm{C} 3-\mathrm{C} 4-\mathrm{C} 4 \mathrm{~A}-\mathrm{C} 5 & -179.75(19) & \mathrm{C} 36-\mathrm{C} 31-\mathrm{C} 32-\mathrm{C} 38 & -177.4(2) \\ \mathrm{C} 3-\mathrm{C} 4-\mathrm{C} 4 \mathrm{~A}-\mathrm{C} 8 \mathrm{~A} & 0.0(3) & \mathrm{C} 31-\mathrm{C} 32-\mathrm{C} 33-\mathrm{C} 34 & 0.2(3) \\ \mathrm{C} 8 \mathrm{~A}-\mathrm{C} 4 \mathrm{~A}-\mathrm{C} 5-\mathrm{C} 6 & 0.0(3) & \mathrm{C} 38-\mathrm{C} 32-\mathrm{C} 33-\mathrm{C} 34 & 177.8(2) \\ \mathrm{C} 4-\mathrm{C} 4 \mathrm{~A}-\mathrm{C} 5-\mathrm{C} 6 & 179.7(2) & \mathrm{C} 32-\mathrm{C} 33-\mathrm{C} 34-\mathrm{C} 35 & -0.1(3) \\ \mathrm{C} 4 \mathrm{~A}-\mathrm{C} 5-\mathrm{C} 6-\mathrm{C} 7 & 0.2(3) & \mathrm{C} 32-\mathrm{C} 33-\mathrm{C} 34-\mathrm{Br} 34 & -177.84(16) \\ \mathrm{C} 4 \mathrm{~A}-\mathrm{C} 5-\mathrm{C} 6-\mathrm{C} 61 & -178.7(2) & \mathrm{C} 33-\mathrm{C} 34-\mathrm{C} 35-\mathrm{C} 36 & -0.1(3) \\ \mathrm{C} 5-\mathrm{C} 6-\mathrm{C} 7-\mathrm{C} 8 & -0.1(3) & \mathrm{B} 34-\mathrm{C} 34-\mathrm{C} 35-\mathrm{C} 36 & 177.58(17) \\ \mathrm{C} 61-\mathrm{C} 6-\mathrm{C} 7-\mathrm{C} 8 & 178.9(2) & \mathrm{C} 34-\mathrm{C} 35-\mathrm{C} 36-\mathrm{C} 31 & 0.3(3) \\ \mathrm{C} 6-\mathrm{C} 7-\mathrm{C} 8-\mathrm{C} 8 \mathrm{~A} & -0.2(4) & \mathrm{O} 31-\mathrm{C} 31-\mathrm{C} 36-\mathrm{C} 35 & -178.2(2) \\ \mathrm{C} 2-\mathrm{N} 1-\mathrm{C} 8 \mathrm{~A}-\mathrm{C} 8 & 180.0(2) & \mathrm{C} 32-\mathrm{C} 31-\mathrm{C} 36-\mathrm{C} 35 & -0.2(3) \\ \mathrm{C} 2-\mathrm{N} 1-\mathrm{C} 8 \mathrm{~A}-\mathrm{C} 4 \mathrm{~A} & 0.2(3) & \mathrm{C} 33-\mathrm{C} 32-\mathrm{C} 38-\mathrm{O} 38 & -18.0(4) \\ \mathrm{C} 7-\mathrm{C} 8-\mathrm{C} 8 \mathrm{~A}-\mathrm{N} 1 & -179.4(2) & \mathrm{C} 31-\mathrm{C} 32-\mathrm{C} 38-\mathrm{O} 38 & 159.5(3) \\ \mathrm{C} 7-\mathrm{C} 8-\mathrm{C} 8 \mathrm{~A}-\mathrm{C} 4 \mathrm{~A} & 0.4(3) & \mathrm{C} 33-\mathrm{C} 32-\mathrm{C} 38-\mathrm{O} 39 & 161.8(2) \\ \mathrm{C} 5-\mathrm{C} 4 \mathrm{~A}-\mathrm{C} 8 \mathrm{~A}-\mathrm{N} 1 & 179.49(19) & \mathrm{C} 31-\mathrm{C} 32-\mathrm{C} 38-\mathrm{O} 39 & -20.7(3) \\ \mathrm{C} 4-\mathrm{C} 4 \mathrm{~A}-\mathrm{C} 8 \mathrm{~A}-\mathrm{N} 1 & -0.3(3) & \mathrm{O} 38-\mathrm{C} 38-\mathrm{O} 39-\mathrm{C} 39 & 3.5(4) \\ \mathrm{C} 5-\mathrm{C} 4 \mathrm{~A}-\mathrm{C} 8 \mathrm{~A}-\mathrm{C} 8 & -0.3(3) & \mathrm{C} 32-\mathrm{C} 38-\mathrm{O} 39-\mathrm{C} 39 & -176.4(2) \\ \mathrm{C} 4-\mathrm{C} 4 \mathrm{~A}-\mathrm{C} 8 \mathrm{~A}-\mathrm{C} 8 & 179.95(19) & & \end{array}$

Hydrogen-bond geometry $\left(\AA,{ }^{\circ}\right)$

\begin{tabular}{lllll}
\hline$D-\mathrm{H} \cdots A$ & $D-\mathrm{H}$ & $\mathrm{H} \cdots A$ & $D \cdots A$ & $D-\mathrm{H} \cdots A$ \\
\hline $\mathrm{C} 36-\mathrm{H} 36 \cdots \mathrm{O} 38^{\mathrm{i}}$ & 0.95 & 2.53 & $3.277(3)$ & 136 \\
\hline
\end{tabular}

Symmetry code: (i) $x+1 / 2, y,-z+1 / 2$.

(III) Methyl 2-[(2-chloro-6-methylquinolin-3-yl)methoxy]benzoate

Crystal data

$\mathrm{C}_{19} \mathrm{H}_{16} \mathrm{ClNO}_{3}$

$M_{r}=341.78$

Orthorhombic, $P 2{ }_{1} 2_{1} 2_{1}$

$a=13.5860$ (3) $\AA$

$b=15.5857$ (2) $\AA$

$c=30.9389(5) \AA$

$V=6551.2(2) \AA^{3}$

$Z=16$

$F(000)=2848$

\section{Data collection}

Agilent Eos Gemini diffractometer

Radiation source: Enhance $(\mathrm{Cu}) \mathrm{X}$-ray Source $\omega$ scans

Absorption correction: multi-scan

(SADABS; Sheldrick, 2003)

$T_{\min }=0.472, T_{\max }=0.734$

45901 measured reflections
$D_{\mathrm{x}}=1.386 \mathrm{Mg} \mathrm{m}^{-3}$

$\mathrm{Cu} K \alpha$ radiation, $\lambda=1.54184 \AA$

Cell parameters from 12841 reflections

$\theta=3.6-72.6^{\circ}$

$\mu=2.21 \mathrm{~mm}^{-1}$

$T=173 \mathrm{~K}$

Block, colourless

$0.48 \times 0.26 \times 0.14 \mathrm{~mm}$

12840 independent reflections

11257 reflections with $I>2 \sigma(I)$

$R_{\text {int }}=0.048$

$\theta_{\max }=72.6^{\circ}, \theta_{\min }=3.6^{\circ}$

$h=-16 \rightarrow 15$

$k=-19 \rightarrow 15$

$l=-38 \rightarrow 30$ 


\section{Refinement}

Refinement on $F^{2}$

Least-squares matrix: full

$R\left[F^{2}>2 \sigma\left(F^{2}\right)\right]=0.046$

$w R\left(F^{2}\right)=0.129$

$S=1.04$

12840 reflections

874 parameters

0 restraints

Hydrogen site location: inferred from neighbouring sites
$\mathrm{H}$-atom parameters constrained

$w=1 /\left[\sigma^{2}\left(F_{\mathrm{o}}{ }^{2}\right)+(0.0759 P)^{2}+0.9452 P\right]$

where $P=\left(F_{\mathrm{o}}^{2}+2 F_{\mathrm{c}}^{2}\right) / 3$

$(\Delta / \sigma)_{\max }=0.001$

$\Delta \rho_{\max }=0.42 \mathrm{e}^{-3}$

$\Delta \rho_{\text {min }}=-0.31$ e $\AA^{-3}$

Absolute structure: Refined as an inversion twin.

Absolute structure parameter: 0.152 (16)

Special details

Geometry. All e.s.d.'s (except the e.s.d. in the dihedral angle between two 1.s. planes) are estimated using the full covariance matrix. The cell e.s.d.'s are taken into account individually in the estimation of e.s.d.'s in distances, angles and torsion angles; correlations between e.s.d.'s in cell parameters are only used when they are defined by crystal symmetry. An approximate (isotropic) treatment of cell e.s.d.'s is used for estimating e.s.d.'s involving 1.s. planes.

Refinement. Refined as a 2-component inversion twin.

Fractional atomic coordinates and isotropic or equivalent isotropic displacement parameters $\left(\hat{A}^{2}\right)$

\begin{tabular}{lllll}
\hline & $x$ & $y$ & $z$ & $U_{\text {iso }} * / U_{\text {eq }}$ \\
\hline N11 & $0.0311(2)$ & $0.26008(18)$ & $0.44748(8)$ & $0.0301(6)$ \\
C12 & $0.0259(3)$ & $0.2694(2)$ & $0.40606(10)$ & $0.0293(7)$ \\
C112 & $0.02044(8)$ & $0.17391(5)$ & $0.37626(3)$ & $0.0430(2)$ \\
C13 & $0.0238(2)$ & $0.3474(2)$ & $0.38287(10)$ & $0.0268(6)$ \\
C14 & $0.0259(2)$ & $0.4211(2)$ & $0.40728(10)$ & $0.0282(7)$ \\
H14 & 0.0256 & 0.4756 & 0.3935 & $0.034^{*}$ \\
C14A & $0.0287(2)$ & $0.4159(2)$ & $0.45268(11)$ & $0.0282(7)$ \\
C15 & $0.0243(3)$ & $0.4892(2)$ & $0.47997(12)$ & $0.0332(7)$ \\
H15 & 0.0226 & 0.5447 & 0.4674 & $0.040^{*}$ \\
C16 & $0.0225(3)$ & $0.4814(3)$ & $0.52403(12)$ & $0.0363(8)$ \\
C17 & $0.0282(3)$ & $0.3981(3)$ & $0.54256(11)$ & $0.0382(8)$ \\
H17 & 0.0286 & 0.3923 & 0.5731 & $0.046^{*}$ \\
C18 & $0.0332(3)$ & $0.3261(2)$ & $0.51731(11)$ & $0.0348(7)$ \\
H18 & 0.0371 & 0.2710 & 0.5304 & $0.042^{*}$ \\
C18A & $0.0325(2)$ & $0.3337(2)$ & $0.47186(11)$ & $0.0290(7)$ \\
C137 & $0.0188(3)$ & $0.3476(2)$ & $0.33422(10)$ & $0.0302(7)$ \\
H13A & 0.0764 & 0.3171 & 0.3220 & $0.036^{*}$ \\
H13B & -0.0418 & 0.3184 & 0.3243 & $0.036^{*}$ \\
O131 & $0.0187(2)$ & $0.43457(15)$ & $0.32062(7)$ & $0.0346(5)$ \\
C131 & $0.0188(3)$ & $0.4508(2)$ & $0.27728(10)$ & $0.0277(7)$ \\
C132 & $0.0243(3)$ & $0.5371(2)$ & $0.26405(10)$ & $0.0284(7)$ \\
C133 & $0.0259(3)$ & $0.5540(2)$ & $0.21978(11)$ & $0.0338(8)$ \\
H133 & 0.0303 & 0.6119 & 0.2103 & $0.041^{*}$ \\
C134 & $0.0215(3)$ & $0.4895(3)$ & $0.18938(11)$ & $0.0404(9)$ \\
H134 & 0.0230 & 0.5027 & 0.1594 & $0.048^{*}$ \\
C135 & $0.0147(3)$ & $0.4051(3)$ & $0.20307(12)$ & $0.0391(8)$ \\
H135 & 0.0107 & 0.3602 & 0.1824 & $0.047^{*}$ \\
& & & &
\end{tabular}




\begin{tabular}{|c|c|c|c|c|}
\hline C136 & $0.0138(3)$ & $0.3858(2)$ & $0.24656(11)$ & $0.0349(8)$ \\
\hline H136 & 0.0097 & 0.3276 & 0.2556 & $0.042 *$ \\
\hline $\mathrm{C} 138$ & $0.0272(3)$ & $0.6138(2)$ & $0.29250(11)$ & $0.0318(7)$ \\
\hline $\mathrm{O} 138$ & $0.0287(3)$ & $0.68600(18)$ & $0.27853(9)$ & $0.0590(8)$ \\
\hline O139 & $0.0286(2)$ & $0.59794(16)$ & $0.33477(8)$ & $0.0362(6)$ \\
\hline C139 & $0.0327(3)$ & $0.6724(3)$ & $0.36214(12)$ & $0.0412(9)$ \\
\hline H19A & 0.0351 & 0.6543 & 0.3925 & $0.062 *$ \\
\hline H19B & -0.0259 & 0.7077 & 0.3574 & $0.062 *$ \\
\hline H19C & 0.0917 & 0.7058 & 0.3553 & $0.062 *$ \\
\hline C161 & $0.0161(4)$ & $0.5585(3)$ & $0.55315(13)$ & $0.0489(10)$ \\
\hline H16A & 0.0696 & 0.5566 & 0.5743 & $0.073^{*}$ \\
\hline H16B & -0.0473 & 0.5583 & 0.5683 & $0.073^{*}$ \\
\hline $\mathrm{H} 16 \mathrm{C}$ & 0.0217 & 0.6109 & 0.5358 & $0.073^{*}$ \\
\hline $\mathrm{N} 21$ & $0.2866(2)$ & 0.67705 (19) & $0.53534(10)$ & $0.0345(6)$ \\
\hline $\mathrm{C} 22$ & $0.2884(3)$ & $0.6675(2)$ & $0.49411(11)$ & $0.0311(7)$ \\
\hline $\mathrm{Cl} 22$ & $0.30603(8)$ & $0.76266(6)$ & $0.46423(3)$ & $0.0463(2)$ \\
\hline $\mathrm{C} 23$ & $0.2803(2)$ & $0.5902(2)$ & $0.47028(11)$ & $0.0297(7)$ \\
\hline $\mathrm{C} 24$ & $0.2724(3)$ & $0.5170(2)$ & $0.49451(11)$ & $0.0312(7)$ \\
\hline $\mathrm{H} 24$ & 0.2675 & 0.4628 & 0.4806 & $0.037^{*}$ \\
\hline $\mathrm{C} 24 \mathrm{~A}$ & $0.2716(3)$ & $0.5217(2)$ & $0.54036(11)$ & $0.0302(7)$ \\
\hline $\mathrm{C} 25$ & $0.2632(3)$ & $0.4488(2)$ & $0.56743(12)$ & $0.0359(8)$ \\
\hline $\mathrm{H} 25$ & 0.2604 & 0.3933 & 0.5548 & $0.043^{*}$ \\
\hline $\mathrm{C} 26$ & $0.2590(3)$ & $0.4568(3)$ & $0.61136(13)$ & $0.0406(9)$ \\
\hline $\mathrm{C} 27$ & $0.2650(3)$ & $0.5396(3)$ & $0.62980(13)$ & $0.0434(9)$ \\
\hline $\mathrm{H} 27$ & 0.2622 & 0.5453 & 0.6603 & $0.052 *$ \\
\hline $\mathrm{C} 28$ & $0.2746(3)$ & $0.6112(3)$ & $0.60521(12)$ & $0.0388(8)$ \\
\hline $\mathrm{H} 28$ & 0.2794 & 0.6660 & 0.6186 & $0.047^{*}$ \\
\hline $\mathrm{C} 28 \mathrm{~A}$ & $0.2774(3)$ & $0.6041(2)$ & $0.55952(12)$ & $0.0332(7)$ \\
\hline C237 & $0.2795(3)$ & $0.5903(2)$ & $0.42186(11)$ & $0.0320(7)$ \\
\hline $\mathrm{H} 23 \mathrm{~A}$ & 0.2233 & 0.6245 & 0.4110 & $0.038^{*}$ \\
\hline H23B & 0.3411 & 0.6158 & 0.4106 & $0.038^{*}$ \\
\hline $\mathrm{O} 231$ & $0.2709(2)$ & $0.50396(16)$ & $0.40813(8)$ & $0.0375(6)$ \\
\hline $\mathrm{C} 231$ & $0.2748(3)$ & $0.4863(2)$ & $0.36514(11)$ & $0.0301(7)$ \\
\hline $\mathrm{C} 232$ & 0.2748 & $0.3993(2)$ & $0.35269(11)$ & $0.0294(7)$ \\
\hline $\mathrm{C} 233$ & $0.2777(3)$ & $0.3806(2)$ & $0.30865(12)$ & $0.0367(8)$ \\
\hline $\mathrm{H} 233$ & 0.2774 & 0.3223 & 0.2998 & $0.044^{*}$ \\
\hline $\mathrm{C} 234$ & $0.2811(3)$ & $0.4434(3)$ & $0.27777(12)$ & $0.0392(9)$ \\
\hline $\mathrm{H} 234$ & 0.2836 & 0.4286 & 0.2480 & $0.047^{*}$ \\
\hline $\mathrm{C} 235$ & $0.2810(3)$ & $0.5288(3)$ & $0.29027(12)$ & $0.0383(8)$ \\
\hline $\mathrm{H} 235$ & 0.2827 & 0.5727 & 0.2690 & $0.046^{*}$ \\
\hline $\mathrm{C} 236$ & $0.2782(3)$ & $0.5503(2)$ & $0.33367(12)$ & $0.0330(7)$ \\
\hline $\mathrm{H} 236$ & 0.2787 & 0.6090 & 0.3421 & $0.040^{*}$ \\
\hline $\mathrm{C} 238$ & $0.2727(3)$ & $0.3238(2)$ & $0.38217(12)$ & $0.0331(8)$ \\
\hline $\mathrm{O} 238$ & $0.2553(3)$ & $0.25274(18)$ & $0.36991(10)$ & $0.0546(8)$ \\
\hline $\mathrm{O} 239$ & $0.2936(2)$ & $0.34248(17)$ & $0.42314(8)$ & $0.0417(6)$ \\
\hline C239 & $0.2981(3)$ & $0.2700(3)$ & $0.45206(13)$ & $0.0485(10)$ \\
\hline $\mathrm{H} 29 \mathrm{~A}$ & 0.3414 & 0.2260 & 0.4398 & $0.073^{*}$ \\
\hline H29B & 0.2319 & 0.2462 & 0.4559 & $0.073 *$ \\
\hline
\end{tabular}




\begin{tabular}{|c|c|c|c|c|}
\hline $\mathrm{H} 29 \mathrm{C}$ & 0.3241 & 0.2885 & 0.4801 & $0.073^{*}$ \\
\hline C261 & $0.2477(4)$ & 0.3792 & $0.64007(14)$ & 0.0528 \\
\hline $\mathrm{H} 26 \mathrm{~A}$ & 0.3075 & 0.3716 & 0.6574 & $0.079 *$ \\
\hline $\mathrm{H} 26 \mathrm{~B}$ & 0.2372 & 0.3281 & 0.6222 & $0.079 *$ \\
\hline $\mathrm{H} 26 \mathrm{C}$ & 0.1912 & 0.3873 & 0.6593 & $0.079 *$ \\
\hline N31 & $0.5271(2)$ & $0.39181(18)$ & $0.44626(9)$ & $0.0282(6)$ \\
\hline $\mathrm{C} 32$ & $0.5293(2)$ & $0.3996(2)$ & $0.40484(10)$ & $0.0272(7)$ \\
\hline $\mathrm{C} 132$ & $0.53360(8)$ & $0.30319(5)$ & $0.37552(3)$ & $0.0403(2)$ \\
\hline C33 & $0.5300(2)$ & $0.4771(2)$ & $0.38069(10)$ & $0.0253(6)$ \\
\hline $\mathrm{C} 34$ & $0.5317(2)$ & $0.5512(2)$ & $0.40443(10)$ & $0.0272(7)$ \\
\hline H34 & 0.5335 & 0.6052 & 0.3902 & $0.033^{*}$ \\
\hline C34A & $0.5309(3)$ & 0.5474 & $0.44993(11)$ & $0.0277(7)$ \\
\hline $\mathrm{C} 35$ & 0.5330 & $0.6216(2)$ & $0.47646(12)$ & $0.0345(8)$ \\
\hline H35 & 0.5362 & 0.6766 & 0.4633 & $0.041 *$ \\
\hline C36 & 0.5305 & $0.6157(3)$ & $0.52091(12)$ & $0.0387(8)$ \\
\hline C37 & 0.5264 & $0.5331(3)$ & $0.53988(11)$ & $0.0401(8)$ \\
\hline H37 & 0.5248 & 0.5285 & 0.5705 & $0.048^{*}$ \\
\hline C38 & 0.5248 & 0.4602 & $0.51574(11)$ & $0.0356(8)$ \\
\hline H38 & 0.5219 & 0.4057 & 0.5295 & $0.043^{*}$ \\
\hline C38A & 0.5275 & $0.4658(2)$ & $0.46987(10)$ & $0.0279(7)$ \\
\hline C337 & 0.5297 & $0.4760(2)$ & $0.33200(10)$ & $0.0261(6)$ \\
\hline $\mathrm{H} 33 \mathrm{~A}$ & 0.5895 & 0.4469 & 0.3210 & $0.031 *$ \\
\hline H33B & 0.4711 & 0.4450 & 0.3211 & $0.031 *$ \\
\hline O331 & $0.5278(2)$ & $0.56258(14)$ & $0.31813(7)$ & $0.0317(5)$ \\
\hline C331 & $0.5256(2)$ & $0.5789(2)$ & $0.27513(10)$ & $0.0265(6)$ \\
\hline C332 & 0.5200 & $0.6657(2)$ & $0.26216(10)$ & $0.0299(7)$ \\
\hline C333 & $0.5204(3)$ & $0.6837(2)$ & $0.21809(11)$ & $0.0368(8)$ \\
\hline H333 & 0.5178 & 0.7418 & 0.2089 & $0.044^{*}$ \\
\hline C334 & 0.5244 & $0.6196(3)$ & $0.18732(11)$ & $0.0417(9)$ \\
\hline H334 & 0.5254 & 0.6336 & 0.1574 & $0.050 *$ \\
\hline C335 & $0.5269(3)$ & $0.5350(2)$ & $0.20050(11)$ & $0.0372(8)$ \\
\hline H335 & 0.5282 & 0.4905 & 0.1795 & $0.045 *$ \\
\hline C336 & 0.5277 & $0.5144(2)$ & $0.24398(11)$ & $0.0316(7)$ \\
\hline H336 & 0.5297 & 0.4560 & 0.2527 & $0.038 *$ \\
\hline C338 & 0.5135 & 0.7414 & $0.29139(11)$ & $0.0339(7)$ \\
\hline O338 & 0.4943 & $0.81240(18)$ & $0.27849(10)$ & $0.0545(8)$ \\
\hline O339 & $0.5325(2)$ & $0.72473(16)$ & $0.33255(8)$ & $0.0430(6)$ \\
\hline C339 & $0.5292(4)$ & 0.7983 & $0.36114(13)$ & $0.0584(12)$ \\
\hline H39A & 0.5762 & 0.8417 & 0.3511 & $0.088^{*}$ \\
\hline Н39B & 0.5466 & 0.7803 & 0.3905 & $0.088^{*}$ \\
\hline H39C & 0.4627 & 0.8226 & 0.3611 & $0.088^{*}$ \\
\hline C361 & $0.5325(4)$ & 0.6949 & $0.54871(13)$ & $0.0520(11)$ \\
\hline H36A & 0.5506 & 0.7446 & 0.5310 & $0.078 *$ \\
\hline H36B & 0.5810 & 0.6875 & 0.5719 & $0.078^{*}$ \\
\hline $\mathrm{H} 36 \mathrm{C}$ & 0.4673 & 0.7043 & 0.5614 & $0.078 *$ \\
\hline N41 & $0.7784(2)$ & $0.67216(19)$ & $0.35785(9)$ & $0.0312(6)$ \\
\hline $\mathrm{C} 42$ & $0.7768(2)$ & $0.6643(2)$ & $0.31639(11)$ & $0.0276(7)$ \\
\hline $\mathrm{C} 142$ & $0.77365(8)$ & $0.76085(5)$ & $0.28715(3)$ & $0.0395(2)$ \\
\hline
\end{tabular}




$\begin{array}{lllll}\text { C43 } & 0.7776(2) & 0.5869(2) & 0.29224(11) & 0.0265(7) \\ \text { C44 } & 0.7803(2) & 0.5131(2) & 0.31580(11) & 0.0283(7) \\ \text { H44 } & 0.7824 & 0.4593 & 0.3014 & 0.034^{*} \\ \text { C44A } & 0.7802(2) & 0.5158(2) & 0.36136(11) & 0.0278(7) \\ \text { C45 } & 0.7793(3) & 0.4418(2) & 0.38798(12) & 0.0338(8) \\ \text { H45 } & 0.7787 & 0.3867 & 0.3749 & 0.041^{*} \\ \text { C46 } & 0.7792(3) & 0.4481(3) & 0.43207(13) & 0.0371(9) \\ \text { C47 } & 0.7801(3) & 0.5302(3) & 0.45138(12) & 0.0393(9) \\ \text { H47 } & 0.7802 & 0.5347 & 0.4820 & 0.047^{*} \\ \text { C48 } & 0.7811(3) & 0.6034(3) & 0.42712(12) & 0.0371(8) \\ \text { H48 } & 0.7825 & 0.6578 & 0.4409 & 0.045^{*} \\ \text { C48A } & 0.7799(2) & 0.5982(2) & 0.38153(10) & 0.0284(7) \\ \text { C437 } & 0.7759(3) & 0.5886(2) & 0.24373(10) & 0.0287(7) \\ \text { H43A } & 0.7172 & 0.6200 & 0.2333 & 0.034^{*} \\ \text { H43B } & 0.8355 & 0.6176 & 0.2325 & 0.034^{*} \\ \text { O431 } & 0.77309(19) & 0.50187(15) & 0.22965(8) & 0.0334(5) \\ \text { C431 } & 0.7656(3) & 0.4859(2) & 0.18670(11) & 0.0294(7) \\ \text { C432 } & 0.7540(2) & 0.4005(2) & 0.17336(11) & 0.0314(7) \\ \text { C433 } & 0.7493(3) & 0.3835(2) & 0.12886(12) & 0.0388(8) \\ \text { H433 } & 0.7435 & 0.3257 & 0.1194 & 0.047^{*} \\ \text { C434 } & 0.7528(3) & 0.4479(3) & 0.09854(12) & 0.0452(10) \\ \text { H434 } & 0.7488 & 0.4348 & 0.0686 & 0.054^{*} \\ \text { C435 } & 0.7621(3) & 0.5321(3) & 0.11217(12) & 0.0420(9) \\ \text { H435 } & 0.7647 & 0.5770 & 0.0915 & 0.050^{*} \\ \text { C436 } & 0.7678(3) & 0.5512(2) & 0.15565(12) & 0.0353(8) \\ \text { H436 } & 0.7732 & 0.6093 & 0.1646 & 0.042^{*} \\ \text { C438 } & 0.7472(3) & 0.3232(2) & 0.20179(12) & 0.0347(8) \\ \text { O438 } & 0.7358(3) & 0.25227(18) & 0.18788(10) & 0.0560(8) \\ \text { O439 } & 0.7541(2) & 0.33994(16) & 0.24416(8) & 0.0404(6) \\ \text { C439 } & 0.7475(4) & 0.2656(3) & 0.27155(13) & 0.0501(10) \\ \text { H49A } & 0.7496 & 0.2835 & 0.3019 & 0.075^{*} \\ \text { H49B } & 0.8030 & 0.2272 & 0.2656 & 0.075^{*} \\ \text { H49C } & 0.6856 & 0.2355 & 0.2659 & 0.075^{*} \\ \text { C461 } & 0.7757(3) & 0.3687(3) & 0.46052(14) & 0.0498(11) \\ \text { H46A } & 0.7075 & 0.3576 & 0.4693 & 0.075^{*} \\ \text { H46B } & 0.8163 & 0.3780 & 0.4863 & 0.075^{*} \\ \text { H46C } & 0.8009 & 0.3193 & 0.4444 & 0.075^{*} \\ & & & & \end{array}$

Atomic displacement parameters $\left(\AA^{2}\right)$

\begin{tabular}{lllllll}
\hline & $U^{11}$ & $U^{22}$ & $U^{33}$ & $U^{12}$ & $U^{13}$ & $U^{23}$ \\
\hline N11 & $0.0385(15)$ & $0.0228(13)$ & $0.0289(13)$ & $0.0008(12)$ & $-0.0018(12)$ & $0.0024(11)$ \\
C12 & $0.0348(17)$ & $0.0208(15)$ & $0.0324(16)$ & $0.0002(14)$ & $-0.0029(14)$ & $-0.0009(13)$ \\
C112 & $0.0731(6)$ & $0.0208(4)$ & $0.0351(4)$ & $-0.0022(4)$ & $-0.0056(4)$ & $-0.0023(3)$ \\
C13 & $0.0278(15)$ & $0.0240(16)$ & $0.0286(16)$ & $0.0016(13)$ & $-0.0003(13)$ & $0.0023(12)$ \\
C14 & $0.0306(16)$ & $0.0225(16)$ & $0.0315(16)$ & $-0.0009(13)$ & $-0.0002(14)$ & $0.0040(13)$ \\
C14A & $0.0254(15)$ & $0.0247(16)$ & $0.0345(17)$ & $-0.0018(13)$ & $-0.0005(13)$ & $-0.0019(13)$ \\
C15 & $0.0321(17)$ & $0.0271(17)$ & $0.0404(19)$ & $0.0011(14)$ & $0.0004(15)$ & $-0.0037(14)$
\end{tabular}




\begin{tabular}{|c|c|c|c|c|c|c|}
\hline $\mathrm{C} 16$ & $0.0306(17)$ & $0.040(2)$ & 0.039 (2) & $-0.0001(16)$ & $-0.0027(15)$ & $-0.0103(16)$ \\
\hline $\mathrm{C} 17$ & $0.0402(19)$ & $0.048(2)$ & $0.0259(16)$ & $-0.0039(18)$ & $-0.0017(15)$ & $-0.0029(15)$ \\
\hline $\mathrm{C} 18$ & $0.0391(19)$ & $0.0345(18)$ & $0.0309(17)$ & $-0.0009(16)$ & $-0.0023(15)$ & $0.0030(15)$ \\
\hline C18A & $0.0266(16)$ & $0.0276(16)$ & $0.0329(17)$ & $-0.0010(13)$ & $-0.0019(13)$ & $0.0001(14)$ \\
\hline C137 & $0.0413(18)$ & $0.0206(15)$ & $0.0287(16)$ & $0.0009(14)$ & $-0.0029(14)$ & $0.0027(13)$ \\
\hline O131 & $0.0548(15)$ & $0.0209(11)$ & $0.0282(12)$ & $0.0041(11)$ & $-0.0016(11)$ & $0.0038(9)$ \\
\hline $\mathrm{C} 131$ & $0.0296(16)$ & $0.0256(16)$ & $0.0279(16)$ & $0.0039(13)$ & $0.0008(13)$ & $0.0048(13)$ \\
\hline $\mathrm{C} 132$ & $0.0284(16)$ & $0.0270(17)$ & $0.0298(17)$ & $0.0045(14)$ & $0.0011(13)$ & $0.0042(13)$ \\
\hline $\mathrm{C} 133$ & $0.0373(18)$ & $0.0323(18)$ & $0.0316(18)$ & $0.0075(15)$ & $0.0034(15)$ & $0.0107(14)$ \\
\hline $\mathrm{C} 134$ & $0.049(2)$ & $0.046(2)$ & $0.0266(17)$ & $0.0104(19)$ & $0.0010(16)$ & $0.0043(16)$ \\
\hline $\mathrm{C} 135$ & $0.049(2)$ & 0.0355 (19) & $0.0326(18)$ & $0.0083(17)$ & $-0.0029(16)$ & $-0.0056(15)$ \\
\hline $\mathrm{C} 136$ & $0.044(2)$ & $0.0251(17)$ & $0.0359(18)$ & $0.0066(15)$ & $0.0004(15)$ & $-0.0002(14)$ \\
\hline $\mathrm{C} 138$ & $0.0357(18)$ & $0.0251(17)$ & $0.0345(17)$ & $0.0045(14)$ & $-0.0010(14)$ & $0.0045(14)$ \\
\hline $\mathrm{O} 138$ & $0.108(3)$ & $0.0250(14)$ & $0.0440(16)$ & $0.0015(16)$ & $-0.0068(17)$ & $0.0038(12)$ \\
\hline O139 & $0.0511(15)$ & $0.0257(12)$ & $0.0318(12)$ & $-0.0007(11)$ & $-0.0024(11)$ & $-0.0004(10)$ \\
\hline C139 & $0.053(2)$ & $0.0323(19)$ & $0.0382(19)$ & $-0.0024(18)$ & $-0.0049(17)$ & $-0.0082(16)$ \\
\hline C161 & $0.054(2)$ & $0.049(2)$ & $0.044(2)$ & $0.003(2)$ & 0.0007 (19) & $-0.0149(19)$ \\
\hline N21 & $0.0440(17)$ & $0.0262(15)$ & $0.0332(15)$ & $0.0035(13)$ & $-0.0030(13)$ & $-0.0036(12)$ \\
\hline $\mathrm{C} 22$ & $0.0377(18)$ & $0.0238(16)$ & $0.0318(17)$ & $0.0025(14)$ & $-0.0026(13)$ & $0.0006(14)$ \\
\hline $\mathrm{Cl} 22$ & $0.0791(7)$ & $0.0235(4)$ & $0.0363(4)$ & $0.0029(4)$ & $-0.0059(4)$ & 0.0019 \\
\hline $\mathrm{C} 23$ & $0.0307(16)$ & $0.0286(17)$ & $0.0297(17)$ & $0.0046(14)$ & $-0.0027(13)$ & $-0.0045(14)$ \\
\hline $\mathrm{C} 24$ & $0.0332(17)$ & $0.0237(16)$ & $0.0366(19)$ & $0.0004(14)$ & $-0.0022(14)$ & $-0.0045(14)$ \\
\hline $\mathrm{C} 24 \mathrm{~A}$ & $0.0281(16)$ & $0.0295(17)$ & $0.0330(18)$ & $-0.0005(14)$ & $-0.0010(14)$ & $-0.0025(14)$ \\
\hline $\mathrm{C} 25$ & $0.0371(19)$ & $0.0314(18)$ & $0.039(2)$ & $-0.0024(15)$ & $-0.0011(15)$ & $0.0001(15)$ \\
\hline $\mathrm{C} 26$ & $0.040(2)$ & $0.045(2)$ & $0.037(2)$ & $-0.0038(17)$ & $-0.0008(16)$ & $0.0092(17)$ \\
\hline $\mathrm{C} 27$ & $0.046(2)$ & $0.054(2)$ & $0.0295(18)$ & $-0.0019(19)$ & $-0.0019(16)$ & $-0.0007(17)$ \\
\hline $\mathrm{C} 28$ & $0.048(2)$ & $0.039(2)$ & $0.0296(18)$ & $0.0000(17)$ & $-0.0002(16)$ & $-0.0060(15)$ \\
\hline C28A & $0.0322(17)$ & $0.0314(18)$ & $0.0359(18)$ & $0.0022(15)$ & $-0.0013(14)$ & $-0.0021(15)$ \\
\hline $\mathrm{C} 237$ & $0.0403(19)$ & $0.0230(16)$ & $0.0328(18)$ & $0.0032(14)$ & $-0.0021(14)$ & $-0.0036(14)$ \\
\hline $\mathrm{O} 231$ & $0.0597(16)$ & $0.0244(12)$ & $0.0285(13)$ & $-0.0032(11)$ & $0.0045(11)$ & $-0.0034(10)$ \\
\hline $\mathrm{C} 231$ & $0.0297(16)$ & $0.0290(17)$ & $0.0316(18)$ & $-0.0023(14)$ & $-0.0020(13)$ & $-0.0035(13)$ \\
\hline $\mathrm{C} 232$ & $0.0286(16)$ & $0.0276(17)$ & $0.0321(17)$ & $-0.0028(14)$ & $-0.0032(13)$ & $-0.0032(14)$ \\
\hline $\mathrm{C} 233$ & $0.042(2)$ & $0.0306(18)$ & $0.0378(19)$ & $-0.0064(16)$ & $-0.0020(16)$ & $-0.0099(15)$ \\
\hline $\mathrm{C} 234$ & $0.048(2)$ & $0.043(2)$ & $0.0268(18)$ & $-0.0062(18)$ & $-0.0065(15)$ & $-0.0023(16)$ \\
\hline $\mathrm{C} 235$ & $0.041(2)$ & $0.040(2)$ & $0.0338(19)$ & $0.0003(16)$ & $-0.0053(15)$ & $0.0082(16)$ \\
\hline $\mathrm{C} 236$ & $0.0348(18)$ & $0.0275(17)$ & $0.0368(19)$ & $0.0012(14)$ & $-0.0039(15)$ & $0.0011(14)$ \\
\hline $\mathrm{C} 238$ & $0.0301(16)$ & $0.0297(18)$ & $0.039(2)$ & $-0.0025(14)$ & $0.0013(14)$ & $-0.0053(15)$ \\
\hline $\mathrm{O} 238$ & $0.088(2)$ & $0.0271(14)$ & $0.0490(17)$ & $-0.0156(14)$ & $-0.0052(15)$ & $-0.0007(13)$ \\
\hline $\mathrm{O} 239$ & $0.0632(18)$ & $0.0287(13)$ & $0.0330(13)$ & $0.0012(12)$ & $-0.0076(12)$ & $0.0028(11)$ \\
\hline C239 & $0.065(3)$ & $0.036(2)$ & $0.045(2)$ & $0.0049(19)$ & $-0.0081(19)$ & $0.0128(18)$ \\
\hline C261 & $0.061(3)$ & 0.049 (3) & $0.048(2)$ & $-0.005(2)$ & $0.001(2)$ & $0.015(2)$ \\
\hline N31 & $0.0335(14)$ & $0.0237(14)$ & $0.0274(13)$ & $-0.0006(12)$ & $-0.0016(11)$ & $0.0024(11)$ \\
\hline $\mathrm{C} 32$ & $0.0324(16)$ & $0.0188(15)$ & $0.0302(16)$ & $-0.0016(13)$ & $-0.0026(14)$ & $-0.0014(13)$ \\
\hline $\mathrm{Cl} 32$ & $0.0723(6)$ & $0.0187(4)$ & $0.0299(4)$ & -0.0005 & $-0.0027(4)$ & $-0.0015(3)$ \\
\hline $\mathrm{C} 33$ & $0.0249(14)$ & $0.0211(15)$ & $0.0300(16)$ & $0.0011(12)$ & $0.0012(13)$ & $0.0023(13)$ \\
\hline $\mathrm{C} 34$ & $0.0304(16)$ & $0.0203(15)$ & $0.0308(16)$ & $0.0017(13)$ & $0.0005(14)$ & $0.0026(12)$ \\
\hline C34A & $0.0262(16)$ & $0.0268(17)$ & $0.0300(17)$ & $0.0016(13)$ & $-0.0013(13)$ & $-0.0033(13)$ \\
\hline $\mathrm{C} 35$ & $0.0377(18)$ & $0.0281(17)$ & $0.0377(19)$ & $0.0022(15)$ & $-0.0022(15)$ & $-0.0068(15)$ \\
\hline
\end{tabular}




\begin{tabular}{|c|c|c|c|c|c|c|}
\hline $\mathrm{C} 36$ & $0.0371(19)$ & $0.043(2)$ & $0.0364(19)$ & $0.0025(17)$ & $-0.0052(15)$ & $-0.0114(16)$ \\
\hline C37 & $0.042(2)$ & $0.053(2)$ & $0.0261(17)$ & $0.0016(18)$ & $-0.0019(16)$ & $-0.0059(16)$ \\
\hline $\mathrm{C} 38$ & $0.0412(19)$ & $0.039(2)$ & $0.0270(17)$ & $0.0004(17)$ & $-0.0025(15)$ & $0.0027(15)$ \\
\hline C38A & $0.0277(16)$ & $0.0291(17)$ & $0.0268(16)$ & $-0.0011(13)$ & $-0.0015(13)$ & $0.0023(13)$ \\
\hline C337 & $0.0336(16)$ & $0.0178(14)$ & $0.0268(16)$ & $0.0008(13)$ & $0.0005(13)$ & $0.0026(12)$ \\
\hline O331 & 0.0517 (14) & $0.0184(11)$ & $0.0251(11)$ & $-0.0017(11)$ & $0.0010(11)$ & $0.0016(9)$ \\
\hline C331 & $0.0296(15)$ & $0.0244(16)$ & $0.0254(15)$ & $-0.0011(13)$ & $-0.0001(13)$ & $0.0033(12)$ \\
\hline C332 & $0.0324(17)$ & $0.0255(16)$ & $0.0319(17)$ & $0.0009(14)$ & $-0.0019(14)$ & $0.0021(13)$ \\
\hline C333 & $0.045(2)$ & $0.0304(18)$ & $0.0345(18)$ & $0.0046(16)$ & $-0.0043(16)$ & $0.0113(14)$ \\
\hline C334 & $0.055(2)$ & $0.045(2)$ & $0.0244(16)$ & $0.007(2)$ & $-0.0042(16)$ & $0.0058(16)$ \\
\hline C335 & $0.045(2)$ & $0.038(2)$ & $0.0295(17)$ & $0.0029(17)$ & $-0.0036(16)$ & $-0.0040(15)$ \\
\hline C336 & $0.0418(19)$ & $0.0226(16)$ & $0.0304(17)$ & $-0.0010(15)$ & $0.0016(15)$ & $0.0016(13)$ \\
\hline C338 & $0.0391(19)$ & $0.0221(16)$ & $0.0406(18)$ & $0.0006(15)$ & $-0.0016(15)$ & $0.0046(14)$ \\
\hline O338 & $0.087(2)$ & $0.0234(14)$ & $0.0528(17)$ & $0.0118(14)$ & $-0.0069(16)$ & $0.0045(12)$ \\
\hline O339 & $0.0746(19)$ & $0.0206(12)$ & $0.0338(13)$ & $0.0021(12)$ & $-0.0089(13)$ & $-0.0035(10)$ \\
\hline C339 & $0.100(4)$ & $0.028(2)$ & $0.047(2)$ & $0.004(2)$ & $-0.007(2)$ & $-0.0121(18)$ \\
\hline C361 & $0.057(3)$ & $0.054(3)$ & $0.045(2)$ & $0.003(2)$ & $-0.003(2)$ & $-0.023(2)$ \\
\hline N41 & $0.0366(16)$ & $0.0262(14)$ & $0.0308(14)$ & $-0.0005(12)$ & $-0.0009(12)$ & $-0.0026(12)$ \\
\hline $\mathrm{C} 42$ & $0.0324(17)$ & $0.0205(15)$ & $0.0298(16)$ & $-0.0022(13)$ & $-0.0016(13)$ & $0.0015(13)$ \\
\hline $\mathrm{Cl} 42$ & $0.0676(6)$ & $0.0203(4)$ & $0.0306(4)$ & $-0.0046(4)$ & $-0.0026(4)$ & 0.0015 \\
\hline $\mathrm{C} 43$ & $0.0280(16)$ & $0.0233(16)$ & $0.0281(16)$ & $-0.0012(13)$ & $-0.0007(13)$ & $0.0007(13)$ \\
\hline $\mathrm{C} 44$ & $0.0307(16)$ & $0.0229(16)$ & $0.0313(17)$ & $0.0003(13)$ & $-0.0012(13)$ & $-0.0051(13)$ \\
\hline C44A & $0.0241(16)$ & $0.0280(17)$ & $0.0314(17)$ & $0.0011(13)$ & $-0.0001(13)$ & $0.0028(14)$ \\
\hline $\mathrm{C} 45$ & $0.0330(18)$ & $0.0291(18)$ & $0.039(2)$ & $0.0034(15)$ & $-0.0007(15)$ & $0.0065(15)$ \\
\hline $\mathrm{C} 46$ & $0.0259(17)$ & $0.047(2)$ & $0.039(2)$ & $0.0050(16)$ & $-0.0011(15)$ & $0.0125(17)$ \\
\hline $\mathrm{C} 47$ & $0.040(2)$ & $0.052(2)$ & $0.0260(17)$ & $0.0009(17)$ & $-0.0003(15)$ & $0.0090(16)$ \\
\hline $\mathrm{C} 48$ & $0.041(2)$ & $0.039(2)$ & $0.0312(18)$ & $0.0008(16)$ & $-0.0028(15)$ & $-0.0050(16)$ \\
\hline C48A & $0.0274(16)$ & $0.0318(17)$ & $0.0260(16)$ & $-0.0013(13)$ & $0.0010(12)$ & $0.0002(14)$ \\
\hline C437 & $0.0382(18)$ & $0.0207(15)$ & $0.0270(16)$ & $0.0016(14)$ & $0.0001(13)$ & $-0.0025(13)$ \\
\hline O431 & $0.0513(15)$ & $0.0210(12)$ & $0.0278(12)$ & $0.0010(11)$ & $-0.0005(11)$ & $-0.0040(9)$ \\
\hline C431 & $0.0322(17)$ & $0.0278(17)$ & $0.0282(16)$ & $0.0004(14)$ & $0.0012(13)$ & $-0.0039(13)$ \\
\hline $\mathrm{C} 432$ & $0.0329(18)$ & $0.0305(18)$ & $0.0307(17)$ & $-0.0020(14)$ & $0.0026(13)$ & $-0.0029(14)$ \\
\hline $\mathrm{C} 433$ & $0.049(2)$ & $0.0322(18)$ & $0.0356(19)$ & $-0.0055(16)$ & $0.0025(16)$ & $-0.0107(16)$ \\
\hline $\mathrm{C} 434$ & $0.061(3)$ & $0.048(2)$ & $0.0262(18)$ & $-0.004(2)$ & $0.0002(17)$ & $-0.0078(16)$ \\
\hline $\mathrm{C} 435$ & $0.056(2)$ & $0.041(2)$ & $0.0294(19)$ & $0.0004(19)$ & $0.0032(17)$ & $0.0040(16)$ \\
\hline $\mathrm{C} 436$ & $0.043(2)$ & $0.0286(17)$ & $0.0338(19)$ & $-0.0036(16)$ & $0.0031(15)$ & $-0.0020(15)$ \\
\hline C438 & $0.0402(19)$ & $0.0263(18)$ & $0.0376(19)$ & $0.0000(15)$ & $-0.0007(14)$ & $-0.0065(14)$ \\
\hline O438 & $0.092(2)$ & $0.0269(14)$ & $0.0488(17)$ & $-0.0053(15)$ & $-0.0008(15)$ & $-0.0079(13)$ \\
\hline O439 & $0.0629(17)$ & $0.0251(12)$ & $0.0332(13)$ & $-0.0066(11)$ & $-0.0010(12)$ & $0.0014(10)$ \\
\hline C439 & $0.078(3)$ & $0.0304(19)$ & $0.042(2)$ & $-0.007(2)$ & $-0.002(2)$ & $0.0069(17)$ \\
\hline C461 & $0.046(2)$ & $0.059(3)$ & $0.045(2)$ & $0.007(2)$ & -0.0015 (19) & $0.026(2)$ \\
\hline
\end{tabular}

Geometric parameters $\left(A,{ }^{\circ}\right)$

\begin{tabular}{llll}
\hline $\mathrm{N} 11-\mathrm{C} 12$ & $1.292(4)$ & $\mathrm{N} 31-\mathrm{C} 32$ & $1.287(4)$ \\
$\mathrm{N} 11-\mathrm{C} 18 \mathrm{~A}$ & $1.373(4)$ & $\mathrm{N} 31-\mathrm{C} 38 \mathrm{~A}$ & $1.365(4)$ \\
$\mathrm{C} 12-\mathrm{C} 13$ & $1.412(4)$ & $\mathrm{C} 32-\mathrm{C} 33$ & $1.420(4)$ \\
$\mathrm{C} 12-\mathrm{C} 12$ & $1.752(3)$ & $\mathrm{C} 32-\mathrm{C} 132$ & $1.756(3)$
\end{tabular}




\begin{tabular}{|c|c|c|c|}
\hline $\mathrm{C} 13-\mathrm{C} 14$ & $1.375(4)$ & $\mathrm{C} 33-\mathrm{C} 34$ & $1.369(4)$ \\
\hline $\mathrm{C} 13-\mathrm{C} 137$ & $1.507(4)$ & $\mathrm{C} 33-\mathrm{C} 337$ & 1.507 (4) \\
\hline $\mathrm{C} 14-\mathrm{C} 14 \mathrm{~A}$ & $1.408(4)$ & $\mathrm{C} 34-\mathrm{C} 34 \mathrm{~A}$ & $1.409(4)$ \\
\hline C14-H14 & 0.9500 & C $34-\mathrm{H} 34$ & 0.9500 \\
\hline $\mathrm{C} 14 \mathrm{~A}-\mathrm{C} 18 \mathrm{~A}$ & $1.414(5)$ & $\mathrm{C} 34 \mathrm{~A}-\mathrm{C} 38 \mathrm{~A}$ & $1.415(5)$ \\
\hline $\mathrm{C} 14 \mathrm{~A}-\mathrm{C} 15$ & $1.421(5)$ & $\mathrm{C} 34 \mathrm{~A}-\mathrm{C} 35$ & $1.418(5)$ \\
\hline $\mathrm{C} 15-\mathrm{C} 16$ & $1.369(5)$ & $\mathrm{C} 35-\mathrm{C} 36$ & $1.379(5)$ \\
\hline C15-H15 & 0.9500 & C $35-\mathrm{H} 35$ & 0.9500 \\
\hline $\mathrm{C} 16-\mathrm{C} 17$ & $1.421(6)$ & $\mathrm{C} 36-\mathrm{C} 37$ & $1.415(6)$ \\
\hline $\mathrm{C} 16-\mathrm{C} 161$ & $1.505(5)$ & $\mathrm{C} 36-\mathrm{C} 361$ & $1.506(5)$ \\
\hline $\mathrm{C} 17-\mathrm{C} 18$ & $1.370(5)$ & $\mathrm{C} 37-\mathrm{C} 38$ & $1.360(5)$ \\
\hline $\mathrm{C} 17-\mathrm{H} 17$ & 0.9500 & C $37-\mathrm{H} 37$ & 0.9500 \\
\hline $\mathrm{C} 18-\mathrm{C} 18 \mathrm{~A}$ & $1.411(5)$ & $\mathrm{C} 38-\mathrm{C} 38 \mathrm{~A}$ & $1.422(5)$ \\
\hline C18-H18 & 0.9500 & C38-H38 & 0.9500 \\
\hline $\mathrm{C} 137-\mathrm{O} 131$ & $1.419(4)$ & $\mathrm{C} 337-\mathrm{O} 331$ & $1.417(4)$ \\
\hline C137-H13A & 0.9900 & С $337-\mathrm{H} 33 \mathrm{~A}$ & 0.9900 \\
\hline C137-H13B & 0.9900 & С $337-$ Н33В & 0.9900 \\
\hline $\mathrm{O} 131-\mathrm{C} 131$ & $1.365(4)$ & O331-C331 & $1.355(4)$ \\
\hline $\mathrm{C} 131-\mathrm{C} 136$ & $1.391(5)$ & C331-C336 & $1.393(5)$ \\
\hline $\mathrm{C} 131-\mathrm{C} 132$ & $1.408(5)$ & $\mathrm{C} 331-\mathrm{C} 332$ & $1.413(4)$ \\
\hline $\mathrm{C} 132-\mathrm{C} 133$ & $1.395(4)$ & C332-C333 & $1.392(5)$ \\
\hline $\mathrm{C} 132-\mathrm{C} 138$ & $1.486(5)$ & C332-C338 & $1.489(5)$ \\
\hline $\mathrm{C} 133-\mathrm{C} 134$ & $1.378(5)$ & C333-C334 & $1.380(5)$ \\
\hline C133-H133 & 0.9500 & С $333-\mathrm{H} 333$ & 0.9500 \\
\hline $\mathrm{C} 134-\mathrm{C} 135$ & $1.386(6)$ & C334-C335 & $1.380(5)$ \\
\hline C134-H134 & 0.9500 & C334-H334 & 0.9500 \\
\hline $\mathrm{C} 135-\mathrm{C} 136$ & $1.379(5)$ & $\mathrm{C} 335-\mathrm{C} 336$ & $1.383(5)$ \\
\hline C135-H135 & 0.9500 & C335-H335 & 0.9500 \\
\hline C136-H136 & 0.9500 & C336-H336 & 0.9500 \\
\hline $\mathrm{C} 138-\mathrm{O} 138$ & $1.205(4)$ & $\mathrm{C} 338-\mathrm{O} 338$ & $1.205(5)$ \\
\hline $\mathrm{C} 138-\mathrm{O} 139$ & $1.331(4)$ & $\mathrm{C} 338-\mathrm{O} 339$ & $1.325(4)$ \\
\hline O139-C139 & $1.437(4)$ & O339-C339 & $1.448(4)$ \\
\hline C139-H19A & 0.9800 & С $339-\mathrm{H} 39 \mathrm{~A}$ & 0.9800 \\
\hline С139-Н19B & 0.9800 & С $339-$ Н39B & 0.9800 \\
\hline C139- H19C & 0.9800 & C339- H39C & 0.9800 \\
\hline $\mathrm{C} 161-\mathrm{H} 16 \mathrm{~A}$ & 0.9800 & С $361-\mathrm{H} 36 \mathrm{~A}$ & 0.9800 \\
\hline $\mathrm{C} 161-\mathrm{H} 16 \mathrm{~B}$ & 0.9800 & С $361-\mathrm{H} 36 \mathrm{~B}$ & 0.9800 \\
\hline $\mathrm{C} 161-\mathrm{H} 16 \mathrm{C}$ & 0.9800 & С $361-\mathrm{H} 36 \mathrm{C}$ & 0.9800 \\
\hline $\mathrm{N} 21-\mathrm{C} 22$ & $1.284(4)$ & $\mathrm{N} 41-\mathrm{C} 42$ & $1.289(4)$ \\
\hline $\mathrm{N} 21-\mathrm{C} 28 \mathrm{~A}$ & $1.367(5)$ & $\mathrm{N} 41-\mathrm{C} 48 \mathrm{~A}$ & $1.366(4)$ \\
\hline $\mathrm{C} 22-\mathrm{C} 23$ & $1.417(5)$ & $\mathrm{C} 42-\mathrm{C} 43$ & $1.419(5)$ \\
\hline $\mathrm{C} 22-\mathrm{Cl} 22$ & $1.764(4)$ & $\mathrm{C} 42-\mathrm{Cl} 42$ & $1.757(3)$ \\
\hline $\mathrm{C} 23-\mathrm{C} 24$ & $1.369(5)$ & $\mathrm{C} 43-\mathrm{C} 44$ & $1.362(5)$ \\
\hline $\mathrm{C} 23-\mathrm{C} 237$ & $1.498(5)$ & $\mathrm{C} 43-\mathrm{C} 437$ & $1.501(4)$ \\
\hline $\mathrm{C} 24-\mathrm{C} 24 \mathrm{~A}$ & $1.420(5)$ & $\mathrm{C} 44-\mathrm{C} 44 \mathrm{~A}$ & $1.410(5)$ \\
\hline $\mathrm{C} 24-\mathrm{H} 24$ & 0.9500 & $\mathrm{C} 44-\mathrm{H} 44$ & 0.9500 \\
\hline $\mathrm{C} 24 \mathrm{~A}-\mathrm{C} 25$ & $1.415(5)$ & $\mathrm{C} 44 \mathrm{~A}-\mathrm{C} 45$ & $1.417(5)$ \\
\hline $\mathrm{C} 24 \mathrm{~A}-\mathrm{C} 28 \mathrm{~A}$ & $1.418(5)$ & $\mathrm{C} 44 \mathrm{~A}-\mathrm{C} 48 \mathrm{~A}$ & $1.429(5)$ \\
\hline
\end{tabular}




\begin{tabular}{|c|c|c|c|}
\hline $\mathrm{C} 25-\mathrm{C} 26$ & $1.366(5)$ & $\mathrm{C} 45-\mathrm{C} 46$ & $1.368(5)$ \\
\hline $\mathrm{C} 25-\mathrm{H} 25$ & 0.9500 & $\mathrm{C} 45-\mathrm{H} 45$ & 0.9500 \\
\hline $\mathrm{C} 26-\mathrm{C} 27$ & $1.413(6)$ & $\mathrm{C} 46-\mathrm{C} 47$ & $1.413(6)$ \\
\hline $\mathrm{C} 26-\mathrm{C} 261$ & $1.509(5)$ & $\mathrm{C} 46-\mathrm{C} 461$ & $1.519(5)$ \\
\hline $\mathrm{C} 27-\mathrm{C} 28$ & $1.357(6)$ & $\mathrm{C} 47-\mathrm{C} 48$ & $1.365(5)$ \\
\hline $\mathrm{C} 27-\mathrm{H} 27$ & 0.9500 & $\mathrm{C} 47-\mathrm{H} 47$ & 0.9500 \\
\hline $\mathrm{C} 28-\mathrm{C} 28 \mathrm{~A}$ & $1.419(5)$ & $\mathrm{C} 48-\mathrm{C} 48 \mathrm{~A}$ & $1.413(5)$ \\
\hline $\mathrm{C} 28-\mathrm{H} 28$ & 0.9500 & $\mathrm{C} 48-\mathrm{H} 48$ & 0.9500 \\
\hline $\mathrm{C} 237-\mathrm{O} 231$ & $1.416(4)$ & $\mathrm{C} 437-\mathrm{O} 431$ & $1.421(4)$ \\
\hline $\mathrm{C} 237-\mathrm{H} 23 \mathrm{~A}$ & 0.9900 & $\mathrm{C} 437-\mathrm{H} 43 \mathrm{~A}$ & 0.9900 \\
\hline $\mathrm{C} 237-\mathrm{H} 23 \mathrm{~B}$ & 0.9900 & C437-H43B & 0.9900 \\
\hline $\mathrm{O} 231-\mathrm{C} 231$ & $1.359(4)$ & $\mathrm{O} 431-\mathrm{C} 431$ & $1.356(4)$ \\
\hline $\mathrm{C} 231-\mathrm{C} 236$ & $1.395(5)$ & $\mathrm{C} 431-\mathrm{C} 436$ & $1.400(5)$ \\
\hline $\mathrm{C} 231-\mathrm{C} 232$ & $1.409(5)$ & $\mathrm{C} 431-\mathrm{C} 432$ & $1.403(5)$ \\
\hline $\mathrm{C} 232-\mathrm{C} 233$ & $1.394(5)$ & $\mathrm{C} 432-\mathrm{C} 433$ & $1.404(5)$ \\
\hline $\mathrm{C} 232-\mathrm{C} 238$ & $1.489(5)$ & $\mathrm{C} 432-\mathrm{C} 438$ & $1.494(5)$ \\
\hline $\mathrm{C} 233-\mathrm{C} 234$ & $1.368(5)$ & $\mathrm{C} 433-\mathrm{C} 434$ & $1.375(6)$ \\
\hline $\mathrm{C} 233-\mathrm{H} 233$ & 0.9500 & C433-H433 & 0.9500 \\
\hline $\mathrm{C} 234-\mathrm{C} 235$ & $1.386(6)$ & $\mathrm{C} 434-\mathrm{C} 435$ & $1.384(6)$ \\
\hline $\mathrm{C} 234-\mathrm{H} 234$ & 0.9500 & C434-H434 & 0.9500 \\
\hline $\mathrm{C} 235-\mathrm{C} 236$ & $1.384(5)$ & $\mathrm{C} 435-\mathrm{C} 436$ & $1.380(5)$ \\
\hline $\mathrm{C} 235-\mathrm{H} 235$ & 0.9500 & $\mathrm{C} 435-\mathrm{H} 435$ & 0.9500 \\
\hline $\mathrm{C} 236-\mathrm{H} 236$ & 0.9500 & $\mathrm{C} 436-\mathrm{H} 436$ & 0.9500 \\
\hline $\mathrm{C} 238-\mathrm{O} 238$ & $1.195(4)$ & $\mathrm{C} 438-\mathrm{O} 438$ & $1.196(5)$ \\
\hline $\mathrm{C} 238-\mathrm{O} 239$ & $1.331(4)$ & $\mathrm{C} 438-\mathrm{O} 439$ & $1.340(4)$ \\
\hline $\mathrm{O} 239-\mathrm{C} 239$ & $1.443(4)$ & $\mathrm{O} 439-\mathrm{C} 439$ & $1.438(4)$ \\
\hline $\mathrm{C} 239-\mathrm{H} 29 \mathrm{~A}$ & 0.9800 & $\mathrm{C} 439-\mathrm{H} 49 \mathrm{~A}$ & 0.9800 \\
\hline $\mathrm{C} 239-\mathrm{H} 29 \mathrm{~B}$ & 0.9800 & C439- H49B & 0.9800 \\
\hline $\mathrm{C} 239-\mathrm{H} 29 \mathrm{C}$ & 0.9800 & $\mathrm{C} 439-\mathrm{H} 49 \mathrm{C}$ & 0.9800 \\
\hline $\mathrm{C} 261-\mathrm{H} 26 \mathrm{~A}$ & 0.9800 & $\mathrm{C} 461-\mathrm{H} 46 \mathrm{~A}$ & 0.9800 \\
\hline $\mathrm{C} 261-\mathrm{H} 26 \mathrm{~B}$ & 0.9800 & $\mathrm{C} 461-\mathrm{H} 46 \mathrm{~B}$ & 0.9800 \\
\hline $\mathrm{C} 261-\mathrm{H} 26 \mathrm{C}$ & 0.9800 & $\mathrm{C} 461-\mathrm{H} 46 \mathrm{C}$ & 0.9800 \\
\hline $\mathrm{C} 12-\mathrm{N} 11-\mathrm{C} 18 \mathrm{~A}$ & $116.9(3)$ & $\mathrm{C} 32-\mathrm{N} 31-\mathrm{C} 38 \mathrm{~A}$ & $116.9(3)$ \\
\hline $\mathrm{N} 11-\mathrm{C} 12-\mathrm{C} 13$ & $127.0(3)$ & $\mathrm{N} 31-\mathrm{C} 32-\mathrm{C} 33$ & $127.2(3)$ \\
\hline $\mathrm{N} 11-\mathrm{C} 12-\mathrm{Cl12}$ & $115.4(3)$ & $\mathrm{N} 31-\mathrm{C} 32-\mathrm{Cl} 32$ & $115.7(2)$ \\
\hline $\mathrm{C} 13-\mathrm{C} 12-\mathrm{C} 112$ & $117.6(2)$ & $\mathrm{C} 33-\mathrm{C} 32-\mathrm{Cl} 32$ & $117.1(2)$ \\
\hline $\mathrm{C} 14-\mathrm{C} 13-\mathrm{C} 12$ & $116.1(3)$ & $\mathrm{C} 34-\mathrm{C} 33-\mathrm{C} 32$ & $115.8(3)$ \\
\hline $\mathrm{C} 14-\mathrm{C} 13-\mathrm{C} 137$ & $123.2(3)$ & $\mathrm{C} 34-\mathrm{C} 33-\mathrm{C} 337$ & $123.1(3)$ \\
\hline $\mathrm{C} 12-\mathrm{C} 13-\mathrm{C} 137$ & $120.7(3)$ & $\mathrm{C} 32-\mathrm{C} 33-\mathrm{C} 337$ & $121.1(3)$ \\
\hline $\mathrm{C} 13-\mathrm{C} 14-\mathrm{C} 14 \mathrm{~A}$ & 120.0 & $\mathrm{C} 33-\mathrm{C} 34-\mathrm{C} 34 \mathrm{~A}$ & $120.0(3)$ \\
\hline $\mathrm{C} 13-\mathrm{C} 14-\mathrm{H} 14$ & 120.0 & $\mathrm{C} 33-\mathrm{C} 34-\mathrm{H} 34$ & 120.0 \\
\hline $\mathrm{C} 14 \mathrm{~A}-\mathrm{C} 14-\mathrm{H} 14$ & 120.0 & $\mathrm{C} 34 \mathrm{~A}-\mathrm{C} 34-\mathrm{H} 34$ & 120.0 \\
\hline $\mathrm{C} 14-\mathrm{C} 14 \mathrm{~A}-\mathrm{C} 18 \mathrm{~A}$ & $118.2(3)$ & $\mathrm{C} 34-\mathrm{C} 34 \mathrm{~A}-\mathrm{C} 38 \mathrm{~A}$ & $118.3(3)$ \\
\hline $\mathrm{C} 14-\mathrm{C} 14 \mathrm{~A}-\mathrm{C} 15$ & $123.1(3)$ & $\mathrm{C} 34-\mathrm{C} 34 \mathrm{~A}-\mathrm{C} 35$ & $123.0(3)$ \\
\hline $\mathrm{C} 18 \mathrm{~A}-\mathrm{C} 14 \mathrm{~A}-\mathrm{C} 15$ & $118.7(3)$ & $\mathrm{C} 38 \mathrm{~A}-\mathrm{C} 34 \mathrm{~A}-\mathrm{C} 35$ & $118.8(3)$ \\
\hline $\mathrm{C} 16-\mathrm{C} 15-\mathrm{C} 14 \mathrm{~A}$ & $121.4(3)$ & $\mathrm{C} 36-\mathrm{C} 35-\mathrm{C} 34 \mathrm{~A}$ & $121.5(3)$ \\
\hline $\mathrm{C} 16-\mathrm{C} 15-\mathrm{H} 15$ & 119.3 & $\mathrm{C} 36-\mathrm{C} 35-\mathrm{H} 35$ & 119.3 \\
\hline
\end{tabular}




\begin{tabular}{|c|c|}
\hline $\mathrm{C} 14 \mathrm{~A}-\mathrm{C} 15-\mathrm{H} 15$ & 119.3 \\
\hline $\mathrm{C} 15-\mathrm{C} 16-\mathrm{C} 17$ & $118.8(3)$ \\
\hline $\mathrm{C} 15-\mathrm{C} 16-\mathrm{C} 161$ & $121.8(4)$ \\
\hline $\mathrm{C} 17-\mathrm{C} 16-\mathrm{C} 161$ & $119.4(3)$ \\
\hline $\mathrm{C} 18-\mathrm{C} 17-\mathrm{C} 16$ & $121.4(3)$ \\
\hline $\mathrm{C} 18-\mathrm{C} 17-\mathrm{H} 17$ & 119.3 \\
\hline $\mathrm{C} 16-\mathrm{C} 17-\mathrm{H} 17$ & 119.3 \\
\hline $\mathrm{C} 17-\mathrm{C} 18-\mathrm{C} 18 \mathrm{~A}$ & $119.9(3)$ \\
\hline $\mathrm{C} 17-\mathrm{C} 18-\mathrm{H} 18$ & 120.0 \\
\hline $\mathrm{C} 18 \mathrm{~A}-\mathrm{C} 18-\mathrm{H} 18$ & 120.0 \\
\hline $\mathrm{N} 11-\mathrm{C} 18 \mathrm{~A}-\mathrm{C} 18$ & $118.5(3)$ \\
\hline $\mathrm{N} 11-\mathrm{C} 18 \mathrm{~A}-\mathrm{C} 14 \mathrm{~A}$ & $121.8(3)$ \\
\hline $\mathrm{C} 18-\mathrm{C} 18 \mathrm{~A}-\mathrm{C} 14 \mathrm{~A}$ & $119.7(3)$ \\
\hline $\mathrm{O} 131-\mathrm{C} 137-\mathrm{C} 13$ & $107.4(3)$ \\
\hline $\mathrm{O} 131-\mathrm{C} 137-\mathrm{H} 13 \mathrm{~A}$ & 110.2 \\
\hline $\mathrm{C} 13-\mathrm{C} 137-\mathrm{H} 13 \mathrm{~A}$ & 110.2 \\
\hline O131-C137-H13B & 110.2 \\
\hline $\mathrm{C} 13-\mathrm{C} 137-\mathrm{H} 13 \mathrm{~B}$ & 110.2 \\
\hline $\mathrm{H} 13 \mathrm{~A}-\mathrm{C} 137-\mathrm{H} 13 \mathrm{~B}$ & 108.5 \\
\hline $\mathrm{C} 131-\mathrm{O} 131-\mathrm{C} 137$ & $117.9(2)$ \\
\hline $\mathrm{O} 131-\mathrm{C} 131-\mathrm{C} 136$ & $122.4(3)$ \\
\hline $\mathrm{O} 131-\mathrm{C} 131-\mathrm{C} 132$ & $117.6(3)$ \\
\hline $\mathrm{C} 136-\mathrm{C} 131-\mathrm{C} 132$ & $120.0(3)$ \\
\hline $\mathrm{C} 133-\mathrm{C} 132-\mathrm{C} 131$ & $117.8(3)$ \\
\hline $\mathrm{C} 133-\mathrm{C} 132-\mathrm{C} 138$ & $115.4(3)$ \\
\hline $\mathrm{C} 131-\mathrm{C} 132-\mathrm{C} 138$ & $126.8(3)$ \\
\hline $\mathrm{C} 134-\mathrm{C} 133-\mathrm{C} 132$ & $122.1(3)$ \\
\hline $\mathrm{C} 134-\mathrm{C} 133-\mathrm{H} 133$ & 119.0 \\
\hline $\mathrm{C} 132-\mathrm{C} 133-\mathrm{H} 133$ & 119.0 \\
\hline $\mathrm{C} 133-\mathrm{C} 134-\mathrm{C} 135$ & $119.2(3)$ \\
\hline $\mathrm{C} 133-\mathrm{C} 134-\mathrm{H} 134$ & 120.4 \\
\hline $\mathrm{C} 135-\mathrm{C} 134-\mathrm{H} 134$ & 120.4 \\
\hline $\mathrm{C} 136-\mathrm{C} 135-\mathrm{C} 134$ & $120.4(3)$ \\
\hline $\mathrm{C} 136-\mathrm{C} 135-\mathrm{H} 135$ & 119.8 \\
\hline $\mathrm{C} 134-\mathrm{C} 135-\mathrm{H} 135$ & 119.8 \\
\hline $\mathrm{C} 135-\mathrm{C} 136-\mathrm{C} 131$ & $120.5(3)$ \\
\hline $\mathrm{C} 135-\mathrm{C} 136-\mathrm{H} 136$ & 119.8 \\
\hline $\mathrm{C} 131-\mathrm{C} 136-\mathrm{H} 136$ & 119.8 \\
\hline $\mathrm{O} 138-\mathrm{C} 138-\mathrm{O} 139$ & $121.7(3)$ \\
\hline $\mathrm{O} 138-\mathrm{C} 138-\mathrm{C} 132$ & $122.6(3)$ \\
\hline $\mathrm{O} 139-\mathrm{C} 138-\mathrm{C} 132$ & $115.6(3)$ \\
\hline $\mathrm{C} 138-\mathrm{O} 139-\mathrm{C} 139$ & $115.4(3)$ \\
\hline $\mathrm{O} 139-\mathrm{C} 139-\mathrm{H} 19 \mathrm{~A}$ & 109.5 \\
\hline O139- & 109.5 \\
\hline $\mathrm{H} 19 \mathrm{~A}-\mathrm{C} 139-\mathrm{H} 19 \mathrm{~B}$ & 109.5 \\
\hline $\mathrm{O} 139-\mathrm{C} 139-\mathrm{H} 19 \mathrm{C}$ & 109.5 \\
\hline $\mathrm{H} 19 \mathrm{~A}-\mathrm{C} 139-\mathrm{H} 19 \mathrm{C}$ & 109.5 \\
\hline & \\
\hline
\end{tabular}

\begin{tabular}{|c|c|}
\hline $\mathrm{C} 34 \mathrm{~A}-\mathrm{C} 35-\mathrm{H} 35$ & 119.3 \\
\hline $\mathrm{C} 35-\mathrm{C} 36-\mathrm{C} 37$ & $118.4(3)$ \\
\hline $\mathrm{C} 35-\mathrm{C} 36-\mathrm{C} 361$ & $120.9(4)$ \\
\hline $\mathrm{C} 37-\mathrm{C} 36-\mathrm{C} 361$ & $120.6(3)$ \\
\hline $\mathrm{C} 38-\mathrm{C} 37-\mathrm{C} 36$ & $122.2(3)$ \\
\hline $\mathrm{C} 38-\mathrm{C} 37-\mathrm{H} 37$ & 118.9 \\
\hline $\mathrm{C} 36-\mathrm{C} 37-\mathrm{H} 37$ & 118.9 \\
\hline $\mathrm{C} 37-\mathrm{C} 38-\mathrm{C} 38 \mathrm{~A}$ & $119.7(4)$ \\
\hline $\mathrm{C} 37-\mathrm{C} 38-\mathrm{H} 38$ & 120.1 \\
\hline $\mathrm{C} 38 \mathrm{~A}-\mathrm{C} 38-\mathrm{H} 38$ & 120.1 \\
\hline $\mathrm{N} 31-\mathrm{C} 38 \mathrm{~A}-\mathrm{C} 34 \mathrm{~A}$ & $121.8(3)$ \\
\hline $\mathrm{N} 31-\mathrm{C} 38 \mathrm{~A}-\mathrm{C} 38$ & $118.8(3)$ \\
\hline $\mathrm{C} 34 \mathrm{~A}-\mathrm{C} 38 \mathrm{~A}-\mathrm{C} 38$ & $119.4(3)$ \\
\hline $\mathrm{O} 331-\mathrm{C} 337-\mathrm{C} 33$ & $107.0(3)$ \\
\hline $\mathrm{O} 331-\mathrm{C} 337-\mathrm{H} 33 \mathrm{~A}$ & 110.3 \\
\hline $\mathrm{C} 33-\mathrm{C} 337-\mathrm{H} 33 \mathrm{~A}$ & 110.3 \\
\hline $\mathrm{O} 331-\mathrm{C} 337-\mathrm{H} 33 \mathrm{~B}$ & 110.3 \\
\hline $\mathrm{C} 33-\mathrm{C} 337-\mathrm{H} 33 \mathrm{~B}$ & 110.3 \\
\hline $\mathrm{H} 33 \mathrm{~A}-\mathrm{C} 337-\mathrm{H} 33 \mathrm{~B}$ & 108.6 \\
\hline $\mathrm{C} 331-\mathrm{O} 331-\mathrm{C} 337$ & $118.5(2)$ \\
\hline $\mathrm{O} 331-\mathrm{C} 331-\mathrm{C} 336$ & $122.9(3)$ \\
\hline $\mathrm{O} 331-\mathrm{C} 331-\mathrm{C} 332$ & $117.4(3)$ \\
\hline C336-C331-C332 & $119.7(3)$ \\
\hline $\mathrm{C} 333-\mathrm{C} 332-\mathrm{C} 331$ & $118.1(3)$ \\
\hline $\mathrm{C} 333-\mathrm{C} 332-\mathrm{C} 338$ & $115.9(3)$ \\
\hline $\mathrm{C} 331-\mathrm{C} 332-\mathrm{C} 338$ & $126.1(3)$ \\
\hline $\mathrm{C} 334-\mathrm{C} 333-\mathrm{C} 332$ & $122.0(3)$ \\
\hline $\mathrm{C} 334-\mathrm{C} 333-\mathrm{H} 333$ & 119.0 \\
\hline $\mathrm{C} 332-\mathrm{C} 333-\mathrm{H} 333$ & 119.0 \\
\hline $\mathrm{C} 335-\mathrm{C} 334-\mathrm{C} 333$ & $119.2(3)$ \\
\hline $\mathrm{C} 335-\mathrm{C} 334-\mathrm{H} 334$ & 120.4 \\
\hline $\mathrm{C} 333-\mathrm{C} 334-\mathrm{H} 334$ & 120.4 \\
\hline $\mathrm{C} 334-\mathrm{C} 335-\mathrm{C} 336$ & $120.6(3)$ \\
\hline C334-C335-H335 & 119.7 \\
\hline $\mathrm{C} 336-\mathrm{C} 335-\mathrm{H} 335$ & 119.7 \\
\hline $\mathrm{C} 335-\mathrm{C} 336-\mathrm{C} 331$ & $120.3(3)$ \\
\hline $\mathrm{C} 335-\mathrm{C} 336-\mathrm{H} 336$ & 119.8 \\
\hline $\mathrm{C} 331-\mathrm{C} 336-\mathrm{H} 336$ & 119.8 \\
\hline $\mathrm{O} 338-\mathrm{C} 338-\mathrm{O} 339$ & $122.7(3)$ \\
\hline $\mathrm{O} 338-\mathrm{C} 338-\mathrm{C} 332$ & $122.6(3)$ \\
\hline $\mathrm{O} 339-\mathrm{C} 338-\mathrm{C} 332$ & $114.6(3)$ \\
\hline $\mathrm{C} 338-\mathrm{O} 339-\mathrm{C} 339$ & $115.2(3)$ \\
\hline $\mathrm{O} 339-\mathrm{C} 339-\mathrm{H} 39 \mathrm{~A}$ & 109.5 \\
\hline $\mathrm{O} 339-\mathrm{C} 339-\mathrm{H} 39 \mathrm{~B}$ & 109.5 \\
\hline $\mathrm{H} 39 \mathrm{~A}-\mathrm{C} 339-\mathrm{H} 39 \mathrm{~B}$ & 109.5 \\
\hline $\mathrm{O} 339-\mathrm{C} 339-\mathrm{H} 39 \mathrm{C}$ & 109.5 \\
\hline $\mathrm{H} 39 \mathrm{~A}-\mathrm{C} 339-\mathrm{H} 39 \mathrm{C}$ & 109.5 \\
\hline $\mathrm{H} 39 \mathrm{~B}-\mathrm{C} 339-\mathrm{H} 39 \mathrm{C}$ & 109.5 \\
\hline
\end{tabular}


C16-C161-H16A

C16-C161-H16B

H16A-C161-H16B

$\mathrm{C} 16-\mathrm{C} 161-\mathrm{H} 16 \mathrm{C}$

H16A-C161-H16C

H16B - C161-H16C

$\mathrm{C} 22-\mathrm{N} 21-\mathrm{C} 28 \mathrm{~A}$

$\mathrm{N} 21-\mathrm{C} 22-\mathrm{C} 23$

$\mathrm{N} 21-\mathrm{C} 22-\mathrm{Cl} 22$

$\mathrm{C} 23-\mathrm{C} 22-\mathrm{Cl} 22$

$\mathrm{C} 24-\mathrm{C} 23-\mathrm{C} 22$

$\mathrm{C} 24-\mathrm{C} 23-\mathrm{C} 237$

$\mathrm{C} 22-\mathrm{C} 23-\mathrm{C} 237$

$\mathrm{C} 23-\mathrm{C} 24-\mathrm{C} 24 \mathrm{~A}$

$\mathrm{C} 23-\mathrm{C} 24-\mathrm{H} 24$

$\mathrm{C} 24 \mathrm{~A}-\mathrm{C} 24-\mathrm{H} 24$

$\mathrm{C} 25-\mathrm{C} 24 \mathrm{~A}-\mathrm{C} 28 \mathrm{~A}$

$\mathrm{C} 25-\mathrm{C} 24 \mathrm{~A}-\mathrm{C} 24$

$\mathrm{C} 28 \mathrm{~A}-\mathrm{C} 24 \mathrm{~A}-\mathrm{C} 24$

$\mathrm{C} 26-\mathrm{C} 25-\mathrm{C} 24 \mathrm{~A}$

$\mathrm{C} 26-\mathrm{C} 25-\mathrm{H} 25$

$\mathrm{C} 24 \mathrm{~A}-\mathrm{C} 25-\mathrm{H} 25$

$\mathrm{C} 25-\mathrm{C} 26-\mathrm{C} 27$

$\mathrm{C} 25-\mathrm{C} 26-\mathrm{C} 261$

$\mathrm{C} 27-\mathrm{C} 26-\mathrm{C} 261$

$\mathrm{C} 28-\mathrm{C} 27-\mathrm{C} 26$

C28-C27-H 27

$\mathrm{C} 26-\mathrm{C} 27-\mathrm{H} 27$

$\mathrm{C} 27-\mathrm{C} 28-\mathrm{C} 28 \mathrm{~A}$

$\mathrm{C} 27-\mathrm{C} 28-\mathrm{H} 28$

$\mathrm{C} 28 \mathrm{~A}-\mathrm{C} 28-\mathrm{H} 28$

$\mathrm{N} 21-\mathrm{C} 28 \mathrm{~A}-\mathrm{C} 24 \mathrm{~A}$

$\mathrm{N} 21-\mathrm{C} 28 \mathrm{~A}-\mathrm{C} 28$

$\mathrm{C} 24 \mathrm{~A}-\mathrm{C} 28 \mathrm{~A}-\mathrm{C} 28$

O231-C237-C23

O231-C237-H23A

$\mathrm{C} 23-\mathrm{C} 237-\mathrm{H} 23 \mathrm{~A}$

O231-C237-H23B

$\mathrm{C} 23-\mathrm{C} 237-\mathrm{H} 23 \mathrm{~B}$

$\mathrm{H} 23 \mathrm{~A}-\mathrm{C} 237-\mathrm{H} 23 \mathrm{~B}$

$\mathrm{C} 231-\mathrm{O} 231-\mathrm{C} 237$

$\mathrm{O} 231-\mathrm{C} 231-\mathrm{C} 236$

$\mathrm{O} 231-\mathrm{C} 231-\mathrm{C} 232$

$\mathrm{C} 236-\mathrm{C} 231-\mathrm{C} 232$

$\mathrm{C} 233-\mathrm{C} 232-\mathrm{C} 231$

$\mathrm{C} 233-\mathrm{C} 232-\mathrm{C} 238$

$\mathrm{C} 231-\mathrm{C} 232-\mathrm{C} 238$

$\mathrm{C} 234-\mathrm{C} 233-\mathrm{C} 232$
109.5

109.5

109.5

109.5

109.5

109.5

116.7 (3)

127.9 (3)

115.2 (3)

116.9 (3)

115.5 (3)

$123.2(3)$

121.3 (3)

120.3 (3)

119.8

119.8

119.0 (3)

123.4 (3)

$117.6(3)$

121.3 (4)

119.4

119.4

118.8 (4)

121.1 (4)

120.0 (4)

122.0 (4)

119.0

119.0

119.8 (4)

120.1

120.1

122.0 (3)

118.9 (3)

119.1 (3)

107.4 (3)

110.2

110.2

110.2

110.2

108.5

$118.9(3)$

$122.7(3)$

117.5 (3)

119.8 (3)

$117.9(3)$

115.7 (3)

126.3 (3)

122.2 (4)
C36-C361-H36A

C36- C361-H36B

H36A-C361-H36B

$\mathrm{C} 36-\mathrm{C} 361-\mathrm{H} 36 \mathrm{C}$

$\mathrm{H} 36 \mathrm{~A}-\mathrm{C} 361-\mathrm{H} 36 \mathrm{C}$

$\mathrm{H} 36 \mathrm{~B}-\mathrm{C} 361-\mathrm{H} 36 \mathrm{C}$

C42-N41-C48A

$\mathrm{N} 41-\mathrm{C} 42-\mathrm{C} 43$

$\mathrm{N} 41-\mathrm{C} 42-\mathrm{C} 142$

$\mathrm{C} 43-\mathrm{C} 42-\mathrm{Cl} 42$

$\mathrm{C} 44-\mathrm{C} 43-\mathrm{C} 42$

$\mathrm{C} 44-\mathrm{C} 43-\mathrm{C} 437$

$\mathrm{C} 42-\mathrm{C} 43-\mathrm{C} 437$

$\mathrm{C} 43-\mathrm{C} 44-\mathrm{C} 44 \mathrm{~A}$

$\mathrm{C} 43-\mathrm{C} 44-\mathrm{H} 44$

$\mathrm{C} 44 \mathrm{~A}-\mathrm{C} 44-\mathrm{H} 44$

$\mathrm{C} 44-\mathrm{C} 44 \mathrm{~A}-\mathrm{C} 45$

$\mathrm{C} 44-\mathrm{C} 44 \mathrm{~A}-\mathrm{C} 48 \mathrm{~A}$

$\mathrm{C} 45-\mathrm{C} 44 \mathrm{~A}-\mathrm{C} 48 \mathrm{~A}$

$\mathrm{C} 46-\mathrm{C} 45-\mathrm{C} 44 \mathrm{~A}$

$\mathrm{C} 46-\mathrm{C} 45-\mathrm{H} 45$

$\mathrm{C} 44 \mathrm{~A}-\mathrm{C} 45-\mathrm{H} 45$

C45-C46-C47

$\mathrm{C} 45-\mathrm{C} 46-\mathrm{C} 461$

$\mathrm{C} 47-\mathrm{C} 46-\mathrm{C} 461$

$\mathrm{C} 48-\mathrm{C} 47-\mathrm{C} 46$

C48-C47-H47

$\mathrm{C} 46-\mathrm{C} 47-\mathrm{H} 47$

$\mathrm{C} 47-\mathrm{C} 48-\mathrm{C} 48 \mathrm{~A}$

$\mathrm{C} 47-\mathrm{C} 48-\mathrm{H} 48$

$\mathrm{C} 48 \mathrm{~A}-\mathrm{C} 48-\mathrm{H} 48$

$\mathrm{N} 41-\mathrm{C} 48 \mathrm{~A}-\mathrm{C} 48$

$\mathrm{N} 41-\mathrm{C} 48 \mathrm{~A}-\mathrm{C} 44 \mathrm{~A}$

$\mathrm{C} 48-\mathrm{C} 48 \mathrm{~A}-\mathrm{C} 44 \mathrm{~A}$

O431-C437-C43

$\mathrm{O} 431-\mathrm{C} 437-\mathrm{H} 43 \mathrm{~A}$

$\mathrm{C} 43-\mathrm{C} 437-\mathrm{H} 43 \mathrm{~A}$

O431- C437-H43B

$\mathrm{C} 43-\mathrm{C} 437-\mathrm{H} 43 \mathrm{~B}$

H43A-C437-H43B

$\mathrm{C} 431-\mathrm{O} 431-\mathrm{C} 437$

$\mathrm{O} 431-\mathrm{C} 431-\mathrm{C} 436$

O431-C431-C432

$\mathrm{C} 436-\mathrm{C} 431-\mathrm{C} 432$

$\mathrm{C} 431-\mathrm{C} 432-\mathrm{C} 433$

$\mathrm{C} 431-\mathrm{C} 432-\mathrm{C} 438$

$\mathrm{C} 433-\mathrm{C} 432-\mathrm{C} 438$

$\mathrm{C} 434-\mathrm{C} 433-\mathrm{C} 432$
109.5

109.5

109.5

109.5

109.5

109.5

$117.0(3)$

$127.2(3)$

$115.6(3)$

$117.2(2)$

$115.9(3)$

123.4 (3)

120.7 (3)

$120.6(3)$

119.7

119.7

$123.8(3)$

$117.6(3)$

$118.5(3)$

$121.5(4)$

119.3

119.3

119.1 (3)

121.4 (4)

119.5 (4)

$121.6(3)$

119.2

119.2

120.1 (4)

119.9

119.9

119.2 (3)

121.7 (3)

119.1 (3)

106.8 (3)

110.4

110.4

110.4

110.4

108.6

118.5 (3)

122.6 (3)

118.1 (3)

119.4 (3)

118.2 (3)

$126.8(3)$

115.0 (3)

$122.0(3)$ 


\begin{tabular}{|c|c|}
\hline $\mathrm{C} 234-\mathrm{C} 233-\mathrm{H} 233$ & 118.9 \\
\hline $\mathrm{C} 232-\mathrm{C} 233-\mathrm{H} 233$ & 118.9 \\
\hline $\mathrm{C} 233-\mathrm{C} 234-\mathrm{C} 235$ & $119.5(3)$ \\
\hline $\mathrm{C} 233-\mathrm{C} 234-\mathrm{H} 234$ & 120.3 \\
\hline $\mathrm{C} 235-\mathrm{C} 234-\mathrm{H} 234$ & 120.3 \\
\hline $\mathrm{C} 236-\mathrm{C} 235-\mathrm{C} 234$ & $120.2(3)$ \\
\hline $\mathrm{C} 236-\mathrm{C} 235-\mathrm{H} 235$ & 119.9 \\
\hline $\mathrm{C} 234-\mathrm{C} 235-\mathrm{H} 235$ & 119.9 \\
\hline $\mathrm{C} 235-\mathrm{C} 236-\mathrm{C} 231$ & $120.3(3)$ \\
\hline $\mathrm{C} 235-\mathrm{C} 236-\mathrm{H} 236$ & 119.8 \\
\hline $\mathrm{C} 231-\mathrm{C} 236-\mathrm{H} 236$ & 119.8 \\
\hline $\mathrm{O} 238-\mathrm{C} 238-\mathrm{O} 239$ & $123.2(4)$ \\
\hline $\mathrm{O} 238-\mathrm{C} 238-\mathrm{C} 232$ & $122.8(3)$ \\
\hline $\mathrm{O} 239-\mathrm{C} 238-\mathrm{C} 232$ & $114.0(3)$ \\
\hline $\mathrm{C} 238-\mathrm{O} 239-\mathrm{C} 239$ & $115.4(3)$ \\
\hline $\mathrm{O} 239-\mathrm{C} 239-\mathrm{H} 29 \mathrm{~A}$ & 109.5 \\
\hline $\mathrm{O} 239-\mathrm{C} 239-\mathrm{H} 29 \mathrm{~B}$ & 109.5 \\
\hline $\mathrm{H} 29 \mathrm{~A}-\mathrm{C} 239-\mathrm{H} 29 \mathrm{~B}$ & 109.5 \\
\hline $\mathrm{O} 239-\mathrm{C} 239-\mathrm{H} 29 \mathrm{C}$ & 109.5 \\
\hline $\mathrm{H} 29 \mathrm{~A}-\mathrm{C} 239-\mathrm{H} 29 \mathrm{C}$ & 109.5 \\
\hline $\mathrm{H} 29 \mathrm{~B}-\mathrm{C} 239-\mathrm{H} 29 \mathrm{C}$ & 109.5 \\
\hline $\mathrm{C} 26-\mathrm{C} 261-\mathrm{H} 26 \mathrm{~A}$ & 109.5 \\
\hline $\mathrm{C} 26-\mathrm{C} 261-\mathrm{H} 26 \mathrm{~B}$ & 109.5 \\
\hline $\mathrm{H} 26 \mathrm{~A}-\mathrm{C} 261-\mathrm{H} 26 \mathrm{~B}$ & 109.5 \\
\hline $\mathrm{C} 26-\mathrm{C} 261-\mathrm{H} 26 \mathrm{C}$ & 109.5 \\
\hline $\mathrm{H} 26 \mathrm{~A}-\mathrm{C} 261-\mathrm{H} 26 \mathrm{C}$ & 109.5 \\
\hline $\mathrm{H} 26 \mathrm{~B}-\mathrm{C} 261-\mathrm{H} 26 \mathrm{C}$ & 109.5 \\
\hline $\mathrm{C} 18 \mathrm{~A}-\mathrm{N} 11-\mathrm{C} 12-\mathrm{C} 13$ & $1.6(6)$ \\
\hline $\mathrm{C} 18 \mathrm{~A}-\mathrm{N} 11-\mathrm{C} 12-\mathrm{C} 12$ & $-178.1(2)$ \\
\hline $\mathrm{N} 11-\mathrm{C} 12-\mathrm{C} 13-\mathrm{C} 14$ & $-1.1(5)$ \\
\hline $\mathrm{C} 112-\mathrm{C} 12-\mathrm{C} 13-\mathrm{C} 14$ & $178.6(3)$ \\
\hline $\mathrm{N} 11-\mathrm{C} 12-\mathrm{C} 13-\mathrm{C} 137$ & $179.3(3)$ \\
\hline $\mathrm{C} 112-\mathrm{C} 12-\mathrm{C} 13-\mathrm{C} 137$ & $-1.1(5)$ \\
\hline $\mathrm{C} 12-\mathrm{C} 13-\mathrm{C} 14-\mathrm{C} 14 \mathrm{~A}$ & $-0.9(5)$ \\
\hline $\mathrm{C} 137-\mathrm{C} 13-\mathrm{C} 14-\mathrm{C} 14 \mathrm{~A}$ & $178.8(3)$ \\
\hline $\mathrm{C} 13-\mathrm{C} 14-\mathrm{C} 14 \mathrm{~A}-\mathrm{C} 18 \mathrm{~A}$ & $2.1(5)$ \\
\hline $\mathrm{C} 13-\mathrm{C} 14-\mathrm{C} 14 \mathrm{~A}-\mathrm{C} 15$ & $-175.6(3)$ \\
\hline $\mathrm{C} 14-\mathrm{C} 14 \mathrm{~A}-\mathrm{C} 15-\mathrm{C} 16$ & $176.9(4)$ \\
\hline $\mathrm{C} 18 \mathrm{~A}-\mathrm{C} 14 \mathrm{~A}-\mathrm{C} 15-\mathrm{C} 16$ & $-0.7(5)$ \\
\hline $\mathrm{C} 14 \mathrm{~A}-\mathrm{C} 15-\mathrm{C} 16-\mathrm{C} 17$ & $1.9(6)$ \\
\hline $\mathrm{C} 14 \mathrm{~A}-\mathrm{C} 15-\mathrm{C} 16-\mathrm{C} 161$ & $-178.9(3)$ \\
\hline $\mathrm{C} 15-\mathrm{C} 16-\mathrm{C} 17-\mathrm{C} 18$ & $-1.5(6)$ \\
\hline $\mathrm{C} 161-\mathrm{C} 16-\mathrm{C} 17-\mathrm{C} 18$ & $179.3(4)$ \\
\hline $\mathrm{C} 16-\mathrm{C} 17-\mathrm{C} 18-\mathrm{C} 18 \mathrm{~A}$ & $-0.1(6)$ \\
\hline $\mathrm{C} 12-\mathrm{N} 11-\mathrm{C} 18 \mathrm{~A}-\mathrm{C} 18$ & $176.9(3)$ \\
\hline $\mathrm{C} 12-\mathrm{N} 11-\mathrm{C} 18 \mathrm{~A}-\mathrm{C} 14 \mathrm{~A}$ & $-0.2(5)$ \\
\hline $\mathrm{C} 17-\mathrm{C} 18-\mathrm{C} 18 \mathrm{~A}-\mathrm{N} 11$ & $-175.8(3)$ \\
\hline
\end{tabular}

119.0

119.0

$119.2(3)$

120.4

120.4

120.5 (4)

119.8

119.8

120.7 (3)

119.6

119.6

122.7 (4)

$122.8(3)$

$114.5(3)$

$114.6(3)$

109.5

109.5

109.5

109.5

109.5

109.5

109.5

109.5

109.5

109.5

109.5

109.5

$-1.5(5)$

$177.6(2)$

$2.3(5)$

$-176.7(3)$

$-178.2(3)$

$2.8(4)$

$-1.1(5)$

179.4 (3)

-0.5 (5)

179.8 (3)

178.9 (3)

$-0.8(6)$

0.4 (6)

-179.9 (4)

-0.1 (6)

-179.8 (4)

0.2 (6)

$-0.4(5)$

$179.8(3)$

1.4 (5) 


\begin{tabular}{|c|c|c|c|}
\hline $\mathrm{C} 17-\mathrm{C} 18-\mathrm{C} 18 \mathrm{~A}-\mathrm{C} 14 \mathrm{~A}$ & $1.3(5)$ & $\mathrm{C} 35-\mathrm{C} 34 \mathrm{~A}-\mathrm{C} 38 \mathrm{~A}-\mathrm{N} 31$ & $-178.9(3)$ \\
\hline $\mathrm{C} 14-\mathrm{C} 14 \mathrm{~A}-\mathrm{C} 18 \mathrm{~A}-\mathrm{N} 11$ & $-1.6(5)$ & $\mathrm{C} 34-\mathrm{C} 34 \mathrm{~A}-\mathrm{C} 38 \mathrm{~A}-\mathrm{C} 38$ & $-178.9(3)$ \\
\hline $\mathrm{C} 15-\mathrm{C} 14 \mathrm{~A}-\mathrm{C} 18 \mathrm{~A}-\mathrm{N} 11$ & $176.2(3)$ & $\mathrm{C} 35-\mathrm{C} 34 \mathrm{~A}-\mathrm{C} 38 \mathrm{~A}-\mathrm{C} 38$ & $0.9(5)$ \\
\hline $\mathrm{C} 14-\mathrm{C} 14 \mathrm{~A}-\mathrm{C} 18 \mathrm{~A}-\mathrm{C} 18$ & $-178.6(3)$ & $\mathrm{C} 37-\mathrm{C} 38-\mathrm{C} 38 \mathrm{~A}-\mathrm{N} 31$ & $179.2(3)$ \\
\hline $\mathrm{C} 15-\mathrm{C} 14 \mathrm{~A}-\mathrm{C} 18 \mathrm{~A}-\mathrm{C} 18$ & $-0.9(5)$ & $\mathrm{C} 37-\mathrm{C} 38-\mathrm{C} 38 \mathrm{~A}-\mathrm{C} 34 \mathrm{~A}$ & $-0.6(6)$ \\
\hline $\mathrm{C} 14-\mathrm{C} 13-\mathrm{C} 137-\mathrm{O} 131$ & $0.9(5)$ & $\mathrm{C} 34-\mathrm{C} 33-\mathrm{C} 337-\mathrm{O} 331$ & $-2.1(4)$ \\
\hline $\mathrm{C} 12-\mathrm{C} 13-\mathrm{C} 137-\mathrm{O} 131$ & $-179.4(3)$ & $\mathrm{C} 32-\mathrm{C} 33-\mathrm{C} 337-\mathrm{O} 331$ & $178.4(3)$ \\
\hline $\mathrm{C} 13-\mathrm{C} 137-\mathrm{O} 131-\mathrm{C} 131$ & $177.2(3)$ & $\mathrm{C} 33-\mathrm{C} 337-\mathrm{O} 331-\mathrm{C} 331$ & $-178.9(3)$ \\
\hline $\mathrm{C} 137-\mathrm{O} 131-\mathrm{C} 131-\mathrm{C} 136$ & $3.5(5)$ & $\mathrm{C} 337-\mathrm{O} 331-\mathrm{C} 331-\mathrm{C} 336$ & $-1.8(5)$ \\
\hline $\mathrm{C} 137-\mathrm{O} 131-\mathrm{C} 131-\mathrm{C} 132$ & $-176.5(3)$ & $\mathrm{C} 337-\mathrm{O} 331-\mathrm{C} 331-\mathrm{C} 332$ & $177.7(3)$ \\
\hline $\mathrm{O} 131-\mathrm{C} 131-\mathrm{C} 132-\mathrm{C} 133$ & $179.1(3)$ & $\mathrm{O} 331-\mathrm{C} 331-\mathrm{C} 332-\mathrm{C} 333$ & $178.2(3)$ \\
\hline $\mathrm{C} 136-\mathrm{C} 131-\mathrm{C} 132-\mathrm{C} 133$ & $-0.9(5)$ & $\mathrm{C} 336-\mathrm{C} 331-\mathrm{C} 332-\mathrm{C} 333$ & $-2.3(5)$ \\
\hline $\mathrm{O} 131-\mathrm{C} 131-\mathrm{C} 132-\mathrm{C} 138$ & $-1.9(5)$ & $\mathrm{O} 331-\mathrm{C} 331-\mathrm{C} 332-\mathrm{C} 338$ & $-1.8(5)$ \\
\hline $\mathrm{C} 136-\mathrm{C} 131-\mathrm{C} 132-\mathrm{C} 138$ & $178.1(3)$ & $\mathrm{C} 336-\mathrm{C} 331-\mathrm{C} 332-\mathrm{C} 338$ & $177.7(3)$ \\
\hline $\mathrm{C} 131-\mathrm{C} 132-\mathrm{C} 133-\mathrm{C} 134$ & $0.6(6)$ & $\mathrm{C} 331-\mathrm{C} 332-\mathrm{C} 333-\mathrm{C} 334$ & $1.1(6)$ \\
\hline $\mathrm{C} 138-\mathrm{C} 132-\mathrm{C} 133-\mathrm{C} 134$ & $-178.6(3)$ & $\mathrm{C} 338-\mathrm{C} 332-\mathrm{C} 333-\mathrm{C} 334$ & $-178.9(4)$ \\
\hline $\mathrm{C} 132-\mathrm{C} 133-\mathrm{C} 134-\mathrm{C} 135$ & $0.3(6)$ & $\mathrm{C} 332-\mathrm{C} 333-\mathrm{C} 334-\mathrm{C} 335$ & $0.8(6)$ \\
\hline $\mathrm{C} 133-\mathrm{C} 134-\mathrm{C} 135-\mathrm{C} 136$ & $-0.9(6)$ & C333-C334-C335-C336 & $-1.5(6)$ \\
\hline $\mathrm{C} 134-\mathrm{C} 135-\mathrm{C} 136-\mathrm{C} 131$ & $0.5(6)$ & $\mathrm{C} 334-\mathrm{C} 335-\mathrm{C} 336-\mathrm{C} 331$ & $0.2(6)$ \\
\hline $\mathrm{O} 131-\mathrm{C} 131-\mathrm{C} 136-\mathrm{C} 135$ & $-179.6(3)$ & $\mathrm{O} 331-\mathrm{C} 331-\mathrm{C} 336-\mathrm{C} 335$ & $-178.9(3)$ \\
\hline $\mathrm{C} 132-\mathrm{C} 131-\mathrm{C} 136-\mathrm{C} 135$ & $0.4(6)$ & $\mathrm{C} 332-\mathrm{C} 331-\mathrm{C} 336-\mathrm{C} 335$ & $1.7(5)$ \\
\hline $\mathrm{C} 133-\mathrm{C} 132-\mathrm{C} 138-\mathrm{O} 138$ & $1.6(6)$ & $\mathrm{C} 333-\mathrm{C} 332-\mathrm{C} 338-\mathrm{O} 338$ & $11.3(6)$ \\
\hline $\mathrm{C} 131-\mathrm{C} 132-\mathrm{C} 138-\mathrm{O} 138$ & $-177.5(4)$ & $\mathrm{C} 331-\mathrm{C} 332-\mathrm{C} 338-\mathrm{O} 338$ & $-168.7(4)$ \\
\hline $\mathrm{C} 133-\mathrm{C} 132-\mathrm{C} 138-\mathrm{O} 139$ & $-178.1(3)$ & $\mathrm{C} 333-\mathrm{C} 332-\mathrm{C} 338-\mathrm{O} 339$ & $-167.4(3)$ \\
\hline $\mathrm{C} 131-\mathrm{C} 132-\mathrm{C} 138-\mathrm{O} 139$ & $2.8(6)$ & $\mathrm{C} 331-\mathrm{C} 332-\mathrm{C} 338-\mathrm{O} 339$ & $12.7(5)$ \\
\hline $\mathrm{O} 138-\mathrm{C} 138-\mathrm{O} 139-\mathrm{C} 139$ & $-0.5(6)$ & $\mathrm{O} 338-\mathrm{C} 338-\mathrm{O} 339-\mathrm{C} 339$ & $-0.3(6)$ \\
\hline $\mathrm{C} 132-\mathrm{C} 138-\mathrm{O} 139-\mathrm{C} 139$ & $179.2(3)$ & $\mathrm{C} 332-\mathrm{C} 338-\mathrm{O} 339-\mathrm{C} 339$ & $178.3(4)$ \\
\hline $\mathrm{C} 28 \mathrm{~A}-\mathrm{N} 21-\mathrm{C} 22-\mathrm{C} 23$ & $1.2(6)$ & $\mathrm{C} 48 \mathrm{~A}-\mathrm{N} 41-\mathrm{C} 42-\mathrm{C} 43$ & $-0.9(5)$ \\
\hline $\mathrm{C} 28 \mathrm{~A}-\mathrm{N} 21-\mathrm{C} 22-\mathrm{Cl} 22$ & $-177.2(3)$ & $\mathrm{C} 48 \mathrm{~A}-\mathrm{N} 41-\mathrm{C} 42-\mathrm{Cl} 42$ & $179.4(2)$ \\
\hline $\mathrm{N} 21-\mathrm{C} 22-\mathrm{C} 23-\mathrm{C} 24$ & $-2.1(5)$ & $\mathrm{N} 41-\mathrm{C} 42-\mathrm{C} 43-\mathrm{C} 44$ & $0.0(5)$ \\
\hline $\mathrm{C} 122-\mathrm{C} 22-\mathrm{C} 23-\mathrm{C} 24$ & $176.4(3)$ & $\mathrm{Cl} 42-\mathrm{C} 42-\mathrm{C} 43-\mathrm{C} 44$ & $179.8(3)$ \\
\hline $\mathrm{N} 21-\mathrm{C} 22-\mathrm{C} 23-\mathrm{C} 237$ & $177.4(3)$ & $\mathrm{N} 41-\mathrm{C} 42-\mathrm{C} 43-\mathrm{C} 437$ & $-179.7(3)$ \\
\hline $\mathrm{C} 122-\mathrm{C} 22-\mathrm{C} 23-\mathrm{C} 237$ & $-4.2(4)$ & $\mathrm{C} 142-\mathrm{C} 42-\mathrm{C} 43-\mathrm{C} 437$ & $0.1(4)$ \\
\hline $\mathrm{C} 22-\mathrm{C} 23-\mathrm{C} 24-\mathrm{C} 24 \mathrm{~A}$ & $0.7(5)$ & $\mathrm{C} 42-\mathrm{C} 43-\mathrm{C} 44-\mathrm{C} 44 \mathrm{~A}$ & $1.3(5)$ \\
\hline $\mathrm{C} 237-\mathrm{C} 23-\mathrm{C} 24-\mathrm{C} 24 \mathrm{~A}$ & $-178.7(3)$ & $\mathrm{C} 437-\mathrm{C} 43-\mathrm{C} 44-\mathrm{C} 44 \mathrm{~A}$ & $-179.0(3)$ \\
\hline $\mathrm{C} 23-\mathrm{C} 24-\mathrm{C} 24 \mathrm{~A}-\mathrm{C} 25$ & $179.7(3)$ & $\mathrm{C} 43-\mathrm{C} 44-\mathrm{C} 44 \mathrm{~A}-\mathrm{C} 45$ & $177.5(3)$ \\
\hline $\mathrm{C} 23-\mathrm{C} 24-\mathrm{C} 24 \mathrm{~A}-\mathrm{C} 28 \mathrm{~A}$ & $1.1(5)$ & $\mathrm{C} 43-\mathrm{C} 44-\mathrm{C} 44 \mathrm{~A}-\mathrm{C} 48 \mathrm{~A}$ & $-1.7(5)$ \\
\hline $\mathrm{C} 28 \mathrm{~A}-\mathrm{C} 24 \mathrm{~A}-\mathrm{C} 25-\mathrm{C} 26$ & $1.0(5)$ & $\mathrm{C} 44-\mathrm{C} 44 \mathrm{~A}-\mathrm{C} 45-\mathrm{C} 46$ & $-180.0(3)$ \\
\hline $\mathrm{C} 24-\mathrm{C} 24 \mathrm{~A}-\mathrm{C} 25-\mathrm{C} 26$ & $-177.5(4)$ & $\mathrm{C} 48 \mathrm{~A}-\mathrm{C} 44 \mathrm{~A}-\mathrm{C} 45-\mathrm{C} 46$ & $-0.8(5)$ \\
\hline $\mathrm{C} 24 \mathrm{~A}-\mathrm{C} 25-\mathrm{C} 26-\mathrm{C} 27$ & $-1.1(6)$ & $\mathrm{C} 44 \mathrm{~A}-\mathrm{C} 45-\mathrm{C} 46-\mathrm{C} 47$ & $-0.1(6)$ \\
\hline $\mathrm{C} 24 \mathrm{~A}-\mathrm{C} 25-\mathrm{C} 26-\mathrm{C} 261$ & $178.6(4)$ & $\mathrm{C} 44 \mathrm{~A}-\mathrm{C} 45-\mathrm{C} 46-\mathrm{C} 461$ & $178.5(3)$ \\
\hline $\mathrm{C} 25-\mathrm{C} 26-\mathrm{C} 27-\mathrm{C} 28$ & $0.1(6)$ & $\mathrm{C} 45-\mathrm{C} 46-\mathrm{C} 47-\mathrm{C} 48$ & $0.1(6)$ \\
\hline $\mathrm{C} 261-\mathrm{C} 26-\mathrm{C} 27-\mathrm{C} 28$ & $-179.6(4)$ & $\mathrm{C} 461-\mathrm{C} 46-\mathrm{C} 47-\mathrm{C} 48$ & $-178.5(4)$ \\
\hline $\mathrm{C} 26-\mathrm{C} 27-\mathrm{C} 28-\mathrm{C} 28 \mathrm{~A}$ & $0.9(6)$ & $\mathrm{C} 46-\mathrm{C} 47-\mathrm{C} 48-\mathrm{C} 48 \mathrm{~A}$ & $0.8(6)$ \\
\hline $\mathrm{C} 22-\mathrm{N} 21-\mathrm{C} 28 \mathrm{~A}-\mathrm{C} 24 \mathrm{~A}$ & $1.0(5)$ & $\mathrm{C} 42-\mathrm{N} 41-\mathrm{C} 48 \mathrm{~A}-\mathrm{C} 48$ & $-179.6(3)$ \\
\hline $\mathrm{C} 22-\mathrm{N} 21-\mathrm{C} 28 \mathrm{~A}-\mathrm{C} 28$ & $-179.7(3)$ & $\mathrm{C} 42-\mathrm{N} 41-\mathrm{C} 48 \mathrm{~A}-\mathrm{C} 44 \mathrm{~A}$ & $0.4(5)$ \\
\hline $\mathrm{C} 25-\mathrm{C} 24 \mathrm{~A}-\mathrm{C} 28 \mathrm{~A}-\mathrm{N} 21$ & $179.3(3)$ & $\mathrm{C} 47-\mathrm{C} 48-\mathrm{C} 48 \mathrm{~A}-\mathrm{N} 41$ & $178.4(3)$ \\
\hline $\mathrm{C} 24-\mathrm{C} 24 \mathrm{~A}-\mathrm{C} 28 \mathrm{~A}-\mathrm{N} 21$ & $-2.1(5)$ & $\mathrm{C} 47-\mathrm{C} 48-\mathrm{C} 48 \mathrm{~A}-\mathrm{C} 44 \mathrm{~A}$ & $-1.6(5)$ \\
\hline
\end{tabular}




$\begin{array}{ll}\mathrm{C} 25-\mathrm{C} 24 \mathrm{~A}-\mathrm{C} 28 \mathrm{~A}-\mathrm{C} 28 & 0.0(5) \\ \mathrm{C} 24-\mathrm{C} 24 \mathrm{~A}-\mathrm{C} 28 \mathrm{~A}-\mathrm{C} 28 & 178.6(3) \\ \mathrm{C} 27-\mathrm{C} 28-\mathrm{C} 28 \mathrm{~A}-\mathrm{N} 21 & 179.7(4) \\ \mathrm{C} 27-\mathrm{C} 28-\mathrm{C} 28 \mathrm{~A}-\mathrm{C} 24 \mathrm{~A} & -1.0(6) \\ \mathrm{C} 24-\mathrm{C} 23-\mathrm{C} 237-\mathrm{O} 231 & -0.8(5) \\ \mathrm{C} 22-\mathrm{C} 23-\mathrm{C} 237-\mathrm{O} 231 & 179.8(3) \\ \mathrm{C} 23-\mathrm{C} 237-\mathrm{O} 231-\mathrm{C} 231 & -175.9(3) \\ \mathrm{C} 237-\mathrm{O} 231-\mathrm{C} 231-\mathrm{C} 236 & -5.8(5) \\ \mathrm{C} 237-\mathrm{O} 231-\mathrm{C} 231-\mathrm{C} 232 & 174.5(3) \\ \mathrm{O} 231-\mathrm{C} 231-\mathrm{C} 232-\mathrm{C} 233 & 179.3(3) \\ \mathrm{C} 236-\mathrm{C} 231-\mathrm{C} 232-\mathrm{C} 233 & -0.3(5) \\ \mathrm{O} 231-\mathrm{C} 231-\mathrm{C} 232-\mathrm{C} 238 & -1.2(5) \\ \mathrm{C} 236-\mathrm{C} 231-\mathrm{C} 232-\mathrm{C} 238 & 179.2(3) \\ \mathrm{C} 231-\mathrm{C} 232-\mathrm{C} 233-\mathrm{C} 234 & 0.3(6) \\ \mathrm{C} 238-\mathrm{C} 232-\mathrm{C} 233-\mathrm{C} 234 & -179.2(3) \\ \mathrm{C} 232-\mathrm{C} 233-\mathrm{C} 234-\mathrm{C} 235 & -0.5(6) \\ \mathrm{C} 233-\mathrm{C} 234-\mathrm{C} 235-\mathrm{C} 236 & 0.6(6) \\ \mathrm{C} 234-\mathrm{C} 235-\mathrm{C} 236-\mathrm{C} 231 & -0.5(6) \\ \mathrm{O} 231-\mathrm{C} 231-\mathrm{C} 236-\mathrm{C} 235 & -179.2(3) \\ \mathrm{C} 232-\mathrm{C} 231-\mathrm{C} 236-\mathrm{C} 235 & 0.4(5) \\ \mathrm{C} 233-\mathrm{C} 232-\mathrm{C} 238-\mathrm{O} 238 & -14.2(5) \\ \mathrm{C} 231-\mathrm{C} 232-\mathrm{C} 238-\mathrm{O} 238 & 166.4(4) \\ \mathrm{C} 233-\mathrm{C} 232-\mathrm{C} 238-\mathrm{O} 239 & 164.7(3) \\ \mathrm{C} 231-\mathrm{C} 232-\mathrm{C} 238-\mathrm{O} 239 & -14.8(5) \\ \mathrm{O} 238-\mathrm{C} 238-\mathrm{O} 239-\mathrm{C} 239 & 2.5(6) \\ \mathrm{C} 232-\mathrm{C} 238-\mathrm{O} 239-\mathrm{C} 239 & -176.4(3) \\ & \\ & \\ \end{array}$

$\begin{array}{ll}\mathrm{C} 44-\mathrm{C} 44 \mathrm{~A}-\mathrm{C} 48 \mathrm{~A}-\mathrm{N} 41 & 0.8(5) \\ \mathrm{C} 45-\mathrm{C} 44 \mathrm{~A}-\mathrm{C} 48 \mathrm{~A}-\mathrm{N} 41 & -178.4(3) \\ \mathrm{C} 44-\mathrm{C} 44 \mathrm{~A}-\mathrm{C} 48 \mathrm{~A}-\mathrm{C} 48 & -179.2(3) \\ \mathrm{C} 45-\mathrm{C} 44 \mathrm{~A}-\mathrm{C} 48 \mathrm{~A}-\mathrm{C} 48 & 1.6(5) \\ \mathrm{C} 44-\mathrm{C} 43-\mathrm{C} 437-\mathrm{O} 431 & 2.7(5) \\ \mathrm{C} 42-\mathrm{C} 43-\mathrm{C} 437-\mathrm{O} 431 & -177.6(3) \\ \mathrm{C} 43-\mathrm{C} 437-\mathrm{O} 431-\mathrm{C} 431 & 176.4(3) \\ \mathrm{C} 437-\mathrm{O} 431-\mathrm{C} 431-\mathrm{C} 436 & 4.8(5) \\ \mathrm{C} 437-\mathrm{O} 431-\mathrm{C} 431-\mathrm{C} 432 & -174.4(3) \\ \mathrm{O} 431-\mathrm{C} 431-\mathrm{C} 432-\mathrm{C} 433 & -178.1(3) \\ \mathrm{C} 436-\mathrm{C} 431-\mathrm{C} 432-\mathrm{C} 433 & 2.7(5) \\ \mathrm{O} 431-\mathrm{C} 431-\mathrm{C} 432-\mathrm{C} 438 & 1.1(5) \\ \mathrm{C} 436-\mathrm{C} 431-\mathrm{C} 432-\mathrm{C} 438 & -178.1(3) \\ \mathrm{C} 431-\mathrm{C} 432-\mathrm{C} 433-\mathrm{C} 434 & -1.9(6) \\ \mathrm{C} 438-\mathrm{C} 432-\mathrm{C} 433-\mathrm{C} 434 & 178.8(4) \\ \mathrm{C} 432-\mathrm{C} 433-\mathrm{C} 434-\mathrm{C} 435 & 0.6(6) \\ \mathrm{C} 433-\mathrm{C} 434-\mathrm{C} 435-\mathrm{C} 436 & -0.1(7) \\ \mathrm{C} 434-\mathrm{C} 435-\mathrm{C} 436-\mathrm{C} 431 & 0.9(6) \\ \mathrm{O} 431-\mathrm{C} 431-\mathrm{C} 436-\mathrm{C} 435 & 178.5(4) \\ \mathrm{C} 432-\mathrm{C} 431-\mathrm{C} 436-\mathrm{C} 435 & -2.3(6) \\ \mathrm{C} 431-\mathrm{C} 432-\mathrm{C} 438-\mathrm{O} 438 & 178.9(4) \\ \mathrm{C} 433-\mathrm{C} 432-\mathrm{C} 438-\mathrm{O} 438 & -1.9(6) \\ \mathrm{C} 431-\mathrm{C} 432-\mathrm{C} 438-\mathrm{O} 439 & -0.7(5) \\ \mathrm{C} 433-\mathrm{C} 432-\mathrm{C} 438-\mathrm{O} 439 & 178.5(3) \\ \mathrm{O} 438-\mathrm{C} 438-\mathrm{O} 439-\mathrm{C} 439 & 0.4(6) \\ \mathrm{C} 432-\mathrm{C} 438-\mathrm{O} 439-\mathrm{C} 439 & -180.0(3) \\ \end{array}$

Hydrogen-bond geometry $\left(A,{ }^{\circ}\right)$

\begin{tabular}{lllll}
\hline$D-\mathrm{H} \cdots A$ & $D-\mathrm{H}$ & $\mathrm{H} \cdots A$ & $D \cdots A$ & $D-\mathrm{H} \cdots A$ \\
\hline $\mathrm{C} 28-\mathrm{H} 28 \cdots \mathrm{N} 41^{\mathrm{i}}$ & 0.95 & 2.63 & $3.565(5)$ & 169 \\
$\mathrm{C} 136-\mathrm{H} 136 \cdots \mathrm{O} 138^{\mathrm{ii}}$ & 0.95 & 2.50 & $3.261(4)$ & 137 \\
$\mathrm{C} 236-\mathrm{H} 236 \cdots \mathrm{O} 438^{\mathrm{iii}}$ & 0.95 & 2.43 & $3.223(4)$ & 141 \\
$\mathrm{C} 336-\mathrm{H} 336 \cdots \mathrm{O} 338^{\mathrm{iv}}$ & 0.95 & 2.46 & $3.238(4)$ & 139 \\
$\mathrm{C} 436-\mathrm{H} 436 \cdots \mathrm{O} 238^{\mathrm{iii}}$ & 0.95 & 2.51 & $3.254(4)$ & 136 \\
$\mathrm{C} 337-\mathrm{H} 33 B \cdots C g 1$ & 0.99 & 2.64 & $3.441(4)$ & 138 \\
$\mathrm{C} 437-\mathrm{H} 43 A \cdots C g 2$ & 0.99 & 2.64 & $3.446(4)$ & 138 \\
\hline
\end{tabular}

Symmetry codes: (i) $x-1 / 2,-y+3 / 2,-z+1$; (ii) $-x, y-1 / 2,-z+1 / 2$; (iii) $-x+1, y+1 / 2,-z+1 / 2$; (iv) $-x+1, y-1 / 2,-z+1 / 2$.

(IV) 2-Chloro-3-[(naphthalen-1-yloxy)methyl]quinoline

Crystal data

$\mathrm{C}_{20} \mathrm{H}_{14} \mathrm{ClNO}$

$M_{r}=319.77$

Monoclinic, $P 2_{1}$

$a=5.3165(3) \AA$

$b=10.5098$ (4) $\AA$

$c=13.6201(7) \AA$

$\beta=98.527(5)^{\circ}$

$V=752.62(6) \AA^{3}$

$Z=2$

$F(000)=332$

$D_{\mathrm{x}}=1.411 \mathrm{Mg} \mathrm{m}^{-3}$

$\mathrm{Cu} K \alpha$ radiation, $\lambda=1.54184 \AA$

Cell parameters from 2014 reflections

$\theta=3.3-72.6^{\circ}$ 
$\mu=2.27 \mathrm{~mm}^{-1}$

$T=173 \mathrm{~K}$

\section{Data collection}

Agilent Eos Gemini diffractometer

Radiation source: Enhance $(\mathrm{Cu}) \mathrm{X}$-ray Source $\omega$ scans

Absorption correction: multi-scan

(SADABS; Sheldrick, 2003)

$T_{\min }=0.551, T_{\max }=0.834$

4606 measured reflections

\section{Refinement}

Refinement on $F^{2}$

Least-squares matrix: full

$R\left[F^{2}>2 \sigma\left(F^{2}\right)\right]=0.033$

$w R\left(F^{2}\right)=0.090$

$S=1.08$

2014 reflections

208 parameters

1 restraint

Hydrogen site location: inferred from neighbouring sites
Needle, colourless

$0.34 \times 0.10 \times 0.08 \mathrm{~mm}$

2014 independent reflections

1938 reflections with $I>2 \sigma(I)$

$R_{\text {int }}=0.029$

$\theta_{\text {max }}=72.6^{\circ}, \theta_{\text {min }}=3.3^{\circ}$

$h=-6 \rightarrow 6$

$k=-8 \rightarrow 12$

$l=-16 \rightarrow 16$

$\mathrm{H}$-atom parameters constrained

$w=1 /\left[\sigma^{2}\left(F_{\mathrm{o}}^{2}\right)+(0.0579 P)^{2}\right]$

where $P=\left(F_{\mathrm{o}}^{2}+2 F_{\mathrm{c}}^{2}\right) / 3$

$(\Delta / \sigma)_{\max }<0.001$

$\Delta \rho_{\max }=0.22 \mathrm{e} \AA^{-3}$

$\Delta \rho_{\min }=-0.19$ e $\AA^{-3}$

Absolute structure: Classical Flack method preferred over Parsons because s.u. lower.

Absolute structure parameter: -0.007 (18)

\section{Special details}

Geometry. All e.s.d.'s (except the e.s.d. in the dihedral angle between two 1.s. planes) are estimated using the full covariance matrix. The cell e.s.d.'s are taken into account individually in the estimation of e.s.d.'s in distances, angles and torsion angles; correlations between e.s.d.'s in cell parameters are only used when they are defined by crystal symmetry. An approximate (isotropic) treatment of cell e.s.d.'s is used for estimating e.s.d.'s involving l.s. planes.

Fractional atomic coordinates and isotropic or equivalent isotropic displacement parameters $\left(\AA^{2}\right)$

\begin{tabular}{lllll}
\hline & $x$ & $y$ & $z$ & $U_{\text {iso }} * / U_{\text {eq }}$ \\
\hline N1 & $0.1678(4)$ & $0.8551(2)$ & $0.36307(18)$ & $0.0324(5)$ \\
C2 & $0.3087(5)$ & $0.8457(3)$ & $0.29394(19)$ & $0.0300(5)$ \\
C12 & $0.25067(12)$ & $0.95854(7)$ & $0.19826(5)$ & $0.04033(19)$ \\
C3 & $0.5025(5)$ & $0.7539(2)$ & $0.28753(19)$ & $0.0291(5)$ \\
C4 & $0.5420(5)$ & $0.6680(3)$ & $0.3636(2)$ & $0.0311(5)$ \\
H4 & 0.6701 & 0.6049 & 0.3641 & $0.037^{*}$ \\
C4A & $0.3943(5)$ & $0.6721(3)$ & $0.4417(2)$ & $0.0301(5)$ \\
C5 & $0.4220(6)$ & $0.5842(3)$ & $0.5210(2)$ & $0.0373(6)$ \\
H5 & 0.5494 & 0.5202 & 0.5244 & $0.045^{*}$ \\
C6 & $0.2686(6)$ & $0.5897(3)$ & $0.5927(2)$ & $0.0392(6)$ \\
H6 & 0.2863 & 0.5284 & 0.6446 & $0.047^{*}$ \\
C7 & $0.0835(5)$ & $0.6863(3)$ & $0.5899(2)$ & $0.0394(6)$ \\
H7 & -0.0216 & 0.6903 & 0.6404 & $0.047^{*}$ \\
C8 & $0.0539(5)$ & $0.7742(3)$ & $0.5151(2)$ & $0.0375(6)$ \\
H8 & -0.0698 & 0.8396 & 0.5145 & $0.045^{*}$ \\
C8A & $0.2063(5)$ & $0.7682(2)$ & $0.4390(2)$ & $0.0301(5)$ \\
C37A & $0.6440(5)$ & $0.7514(3)$ & $0.20041(19)$ & $0.0309(5)$ \\
H37A & 0.5238 & 0.7395 & 0.1383 & $0.037^{*}$
\end{tabular}




$\begin{array}{lllll}\text { H37B } & 0.7351 & 0.8329 & 0.1958 & 0.037^{*} \\ \text { O31 } & 0.8208(4) & 0.64868(19) & 0.21392(13) & 0.0335(4) \\ \text { C31 } & 0.9563(5) & 0.6249(3) & 0.13811(19) & 0.0300(5) \\ \text { C32 } & 0.9293(5) & 0.6908(3) & 0.0506(2) & 0.0338(6) \\ \text { H32 } & 0.8081 & 0.7576 & 0.0391 & 0.041^{*} \\ \text { C33 } & 1.0815(5) & 0.6599(3) & -0.0230(2) & 0.0359(6) \\ \text { H33 } & 1.0579 & 0.7047 & -0.0842 & 0.043^{*} \\ \text { C34 } & 1.2606(5) & 0.5671(3) & -0.0073(2) & 0.0372(6) \\ \text { H34 } & 1.3650 & 0.5496 & -0.0566 & 0.045^{*} \\ \text { C34A } & 1.2927(5) & 0.4962(2) & 0.0824(2) & 0.0323(6) \\ \text { C35 } & 1.4795(5) & 0.3997(3) & 0.1028(2) & 0.0375(6) \\ \text { H35 } & 1.5873 & 0.3812 & 0.0550 & 0.045^{*} \\ \text { C36 } & 1.5078(5) & 0.3334(3) & 0.1892(2) & 0.0417(7) \\ \text { H36 } & 1.6336 & 0.2687 & 0.2007 & 0.050^{*} \\ \text { C37 } & 1.3522(5) & 0.3594(3) & 0.2622(2) & 0.0386(6) \\ \text { H37 } & 1.3739 & 0.3127 & 0.3226 & 0.046^{*} \\ \text { C38 } & 1.1692(5) & 0.4525(3) & 0.24564(19) & 0.0329(5) \\ \text { H38 } & 1.0628 & 0.4690 & 0.2944 & 0.040^{*} \\ \text { C38A } & 1.1379(5) & 0.5238(2) & 0.1565(2) & 0.0288(5)\end{array}$

Atomic displacement parameters $\left(\AA^{2}\right)$

\begin{tabular}{lllllll}
\hline & $U^{11}$ & $U^{22}$ & $U^{33}$ & $U^{12}$ & $U^{13}$ & $U^{23}$ \\
\hline $\mathrm{N} 1$ & $0.0306(11)$ & $0.0295(11)$ & $0.0363(11)$ & $0.0035(9)$ & $0.0022(9)$ & $-0.0015(9)$ \\
$\mathrm{C} 2$ & $0.0298(12)$ & $0.0270(12)$ & $0.0313(13)$ & $-0.0002(10)$ & $-0.0016(10)$ & $0.0006(10)$ \\
$\mathrm{C} 2$ & $0.0429(3)$ & $0.0359(3)$ & $0.0417(3)$ & $0.0079(3)$ & $0.0048(2)$ & $0.0091(3)$ \\
$\mathrm{C} 3$ & $0.0259(12)$ & $0.0279(12)$ & $0.0319(12)$ & $-0.0018(10)$ & $-0.0011(10)$ & $-0.0042(10)$ \\
$\mathrm{C} 4$ & $0.0276(12)$ & $0.0281(12)$ & $0.0365(13)$ & $0.0036(10)$ & $0.0016(10)$ & $-0.0036(11)$ \\
$\mathrm{C} 4 \mathrm{~A}$ & $0.0268(11)$ & $0.0267(12)$ & $0.0355(13)$ & $-0.0020(10)$ & $0.0007(10)$ & $-0.0045(10)$ \\
$\mathrm{C} 5$ & $0.0376(13)$ & $0.0330(14)$ & $0.0403(14)$ & $0.0040(11)$ & $0.0029(11)$ & $0.0025(11)$ \\
$\mathrm{C} 6$ & $0.0407(15)$ & $0.0389(16)$ & $0.0369(14)$ & $-0.0042(13)$ & $0.0023(12)$ & $0.0053(12)$ \\
$\mathrm{C} 7$ & $0.0346(14)$ & $0.0493(17)$ & $0.0356(14)$ & $-0.0025(13)$ & $0.0090(11)$ & $-0.0027(12)$ \\
$\mathrm{C} 8$ & $0.0321(14)$ & $0.0393(15)$ & $0.0410(15)$ & $0.0034(12)$ & $0.0050(11)$ & $-0.0038(12)$ \\
$\mathrm{C} 8 \mathrm{~A}$ & $0.0265(11)$ & $0.0286(12)$ & $0.0339(13)$ & $-0.0017(10)$ & $0.0006(10)$ & $-0.0046(10)$ \\
$\mathrm{C} 37 \mathrm{~A}$ & $0.0287(12)$ & $0.0285(12)$ & $0.0348(13)$ & $0.0013(10)$ & $0.0024(10)$ & $0.0010(10)$ \\
O31 & $0.0363(9)$ & $0.0332(10)$ & $0.0320(9)$ & $0.0074(8)$ & $0.0078(7)$ & $0.0022(7)$ \\
$\mathrm{C} 31$ & $0.0281(12)$ & $0.0308(12)$ & $0.0313(12)$ & $-0.0043(10)$ & $0.0053(10)$ & $-0.0024(10)$ \\
$\mathrm{C} 32$ & $0.0327(13)$ & $0.0349(14)$ & $0.0331(13)$ & $0.0000(11)$ & $0.0027(10)$ & $-0.0005(11)$ \\
$\mathrm{C} 33$ & $0.0386(14)$ & $0.0397(15)$ & $0.0287(13)$ & $-0.0083(12)$ & $0.0031(11)$ & $0.0006(11)$ \\
$\mathrm{C} 34$ & $0.0365(14)$ & $0.0416(15)$ & $0.0348(14)$ & $-0.0083(12)$ & $0.0095(11)$ & $-0.0087(12)$ \\
$\mathrm{C} 34 \mathrm{~A}$ & $0.0290(12)$ & $0.0316(14)$ & $0.0355(13)$ & $-0.0061(10)$ & $0.0024(10)$ & $-0.0088(10)$ \\
$\mathrm{C} 35$ & $0.0298(13)$ & $0.0373(14)$ & $0.0466(15)$ & $-0.0011(11)$ & $0.0095(11)$ & $-0.0108(12)$ \\
C36 & $0.0313(14)$ & $0.0358(15)$ & $0.0571(18)$ & $0.0044(12)$ & $0.0033(13)$ & $-0.0027(13)$ \\
C37 & $0.0358(14)$ & $0.0366(14)$ & $0.0423(15)$ & $0.0008(12)$ & $0.0023(12)$ & $0.0058(13)$ \\
C38 & $0.0301(11)$ & $0.0324(13)$ & $0.0367(12)$ & $-0.0015(12)$ & $0.0063(10)$ & $-0.0015(13)$ \\
C38A & $0.0259(11)$ & $0.0270(12)$ & $0.0327(12)$ & $-0.0043(9)$ & $0.0013(10)$ & $-0.0039(10)$ \\
& & & & & & \\
\hline & & & & & &
\end{tabular}


Geometric parameters $\left(\AA,{ }^{\circ}\right)$

\begin{tabular}{|c|c|c|c|}
\hline $\mathrm{N} 1-\mathrm{C} 2$ & $1.291(3)$ & $\mathrm{C} 37 \mathrm{~A}-\mathrm{H} 37 \mathrm{~B}$ & 0.9900 \\
\hline $\mathrm{N} 1-\mathrm{C} 8 \mathrm{~A}$ & $1.372(3)$ & $\mathrm{O} 31-\mathrm{C} 31$ & $1.367(3)$ \\
\hline $\mathrm{C} 2-\mathrm{C} 3$ & $1.424(4)$ & $\mathrm{C} 31-\mathrm{C} 32$ & $1.368(4)$ \\
\hline $\mathrm{C} 2-\mathrm{Cl} 2$ & $1.755(3)$ & $\mathrm{C} 31-\mathrm{C} 38 \mathrm{~A}$ & $1.432(4)$ \\
\hline $\mathrm{C} 3-\mathrm{C} 4$ & $1.367(4)$ & $\mathrm{C} 32-\mathrm{C} 33$ & $1.416(4)$ \\
\hline $\mathrm{C} 3-\mathrm{C} 37 \mathrm{~A}$ & $1.497(3)$ & $\mathrm{C} 32-\mathrm{H} 32$ & 0.9500 \\
\hline $\mathrm{C} 4-\mathrm{C} 4 \mathrm{~A}$ & $1.413(3)$ & $\mathrm{C} 33-\mathrm{C} 34$ & $1.357(5)$ \\
\hline $\mathrm{C} 4-\mathrm{H} 4$ & 0.9500 & $\mathrm{C} 33-\mathrm{H} 33$ & 0.9500 \\
\hline $\mathrm{C} 4 \mathrm{~A}-\mathrm{C} 5$ & $1.413(4)$ & C34-C34A & $1.420(4)$ \\
\hline $\mathrm{C} 4 \mathrm{~A}-\mathrm{C} 8 \mathrm{~A}$ & $1.418(4)$ & $\mathrm{C} 34-\mathrm{H} 34$ & 0.9500 \\
\hline $\mathrm{C} 5-\mathrm{C} 6$ & $1.364(4)$ & $\mathrm{C} 34 \mathrm{~A}-\mathrm{C} 35$ & $1.417(4)$ \\
\hline $\mathrm{C} 5-\mathrm{H} 5$ & 0.9500 & $\mathrm{C} 34 \mathrm{~A}-\mathrm{C} 38 \mathrm{~A}$ & $1.423(4)$ \\
\hline $\mathrm{C} 6-\mathrm{C} 7$ & $1.410(4)$ & $\mathrm{C} 35-\mathrm{C} 36$ & $1.357(4)$ \\
\hline $\mathrm{C} 6-\mathrm{H} 6$ & 0.9500 & $\mathrm{C} 35-\mathrm{H} 35$ & 0.9500 \\
\hline $\mathrm{C} 7-\mathrm{C} 8$ & $1.366(4)$ & $\mathrm{C} 36-\mathrm{C} 37$ & $1.411(4)$ \\
\hline $\mathrm{C} 7-\mathrm{H} 7$ & 0.9500 & $\mathrm{C} 36-\mathrm{H} 36$ & 0.9500 \\
\hline $\mathrm{C} 8-\mathrm{C} 8 \mathrm{~A}$ & $1.409(4)$ & $\mathrm{C} 37-\mathrm{C} 38$ & $1.375(4)$ \\
\hline $\mathrm{C} 8-\mathrm{H} 8$ & 0.9500 & C37-H37 & 0.9500 \\
\hline $\mathrm{C} 37 \mathrm{~A}-\mathrm{O} 31$ & $1.426(3)$ & $\mathrm{C} 38-\mathrm{C} 38 \mathrm{~A}$ & $1.416(4)$ \\
\hline $\mathrm{C} 37 \mathrm{~A}-\mathrm{H} 37 \mathrm{~A}$ & 0.9900 & $\mathrm{C} 38-\mathrm{H} 38$ & 0.9500 \\
\hline $\mathrm{C} 2-\mathrm{N} 1-\mathrm{C} 8 \mathrm{~A}$ & $117.4(2)$ & $\mathrm{H} 37 \mathrm{~A}-\mathrm{C} 37 \mathrm{~A}-\mathrm{H} 37 \mathrm{~B}$ & 108.4 \\
\hline $\mathrm{N} 1-\mathrm{C} 2-\mathrm{C} 3$ & $126.9(2)$ & $\mathrm{C} 31-\mathrm{O} 31-\mathrm{C} 37 \mathrm{~A}$ & $116.9(2)$ \\
\hline $\mathrm{N} 1-\mathrm{C} 2-\mathrm{Cl} 2$ & $115.61(19)$ & $\mathrm{O} 31-\mathrm{C} 31-\mathrm{C} 32$ & $124.5(2)$ \\
\hline $\mathrm{C} 3-\mathrm{C} 2-\mathrm{Cl} 2$ & $117.5(2)$ & $\mathrm{O} 31-\mathrm{C} 31-\mathrm{C} 38 \mathrm{~A}$ & $114.9(2)$ \\
\hline $\mathrm{C} 4-\mathrm{C} 3-\mathrm{C} 2$ & $115.6(2)$ & $\mathrm{C} 32-\mathrm{C} 31-\mathrm{C} 38 \mathrm{~A}$ & $120.6(2)$ \\
\hline $\mathrm{C} 4-\mathrm{C} 3-\mathrm{C} 37 \mathrm{~A}$ & $123.4(2)$ & $\mathrm{C} 31-\mathrm{C} 32-\mathrm{C} 33$ & $120.2(3)$ \\
\hline $\mathrm{C} 2-\mathrm{C} 3-\mathrm{C} 37 \mathrm{~A}$ & $120.9(2)$ & $\mathrm{C} 31-\mathrm{C} 32-\mathrm{H} 32$ & 119.9 \\
\hline $\mathrm{C} 3-\mathrm{C} 4-\mathrm{C} 4 \mathrm{~A}$ & $120.7(2)$ & $\mathrm{C} 33-\mathrm{C} 32-\mathrm{H} 32$ & 119.9 \\
\hline $\mathrm{C} 3-\mathrm{C} 4-\mathrm{H} 4$ & 119.7 & $\mathrm{C} 34-\mathrm{C} 33-\mathrm{C} 32$ & $121.0(3)$ \\
\hline $\mathrm{C} 4 \mathrm{~A}-\mathrm{C} 4-\mathrm{H} 4$ & 119.7 & $\mathrm{C} 34-\mathrm{C} 33-\mathrm{H} 33$ & 119.5 \\
\hline $\mathrm{C} 4-\mathrm{C} 4 \mathrm{~A}-\mathrm{C} 5$ & $123.3(2)$ & $\mathrm{C} 32-\mathrm{C} 33-\mathrm{H} 33$ & 119.5 \\
\hline $\mathrm{C} 4-\mathrm{C} 4 \mathrm{~A}-\mathrm{C} 8 \mathrm{~A}$ & $118.1(2)$ & $\mathrm{C} 33-\mathrm{C} 34-\mathrm{C} 34 \mathrm{~A}$ & $120.3(2)$ \\
\hline $\mathrm{C} 5-\mathrm{C} 4 \mathrm{~A}-\mathrm{C} 8 \mathrm{~A}$ & $118.6(2)$ & $\mathrm{C} 33-\mathrm{C} 34-\mathrm{H} 34$ & 119.9 \\
\hline $\mathrm{C} 6-\mathrm{C} 5-\mathrm{C} 4 \mathrm{~A}$ & $120.9(3)$ & $\mathrm{C} 34 \mathrm{~A}-\mathrm{C} 34-\mathrm{H} 34$ & 119.9 \\
\hline $\mathrm{C} 6-\mathrm{C} 5-\mathrm{H} 5$ & 119.5 & $\mathrm{C} 35-\mathrm{C} 34 \mathrm{~A}-\mathrm{C} 34$ & $122.4(3)$ \\
\hline $\mathrm{C} 4 \mathrm{~A}-\mathrm{C} 5-\mathrm{H} 5$ & 119.5 & $\mathrm{C} 35-\mathrm{C} 34 \mathrm{~A}-\mathrm{C} 38 \mathrm{~A}$ & $118.0(3)$ \\
\hline $\mathrm{C} 5-\mathrm{C} 6-\mathrm{C} 7$ & $120.0(3)$ & $\mathrm{C} 34-\mathrm{C} 34 \mathrm{~A}-\mathrm{C} 38 \mathrm{~A}$ & $119.6(3)$ \\
\hline $\mathrm{C} 5-\mathrm{C} 6-\mathrm{H} 6$ & 120.0 & $\mathrm{C} 36-\mathrm{C} 35-\mathrm{C} 34 \mathrm{~A}$ & $121.5(3)$ \\
\hline $\mathrm{C} 7-\mathrm{C} 6-\mathrm{H} 6$ & 120.0 & $\mathrm{C} 36-\mathrm{C} 35-\mathrm{H} 35$ & 119.3 \\
\hline $\mathrm{C} 8-\mathrm{C} 7-\mathrm{C} 6$ & $120.7(3)$ & $\mathrm{C} 34 \mathrm{~A}-\mathrm{C} 35-\mathrm{H} 35$ & 119.3 \\
\hline $\mathrm{C} 8-\mathrm{C} 7-\mathrm{H} 7$ & 119.7 & $\mathrm{C} 35-\mathrm{C} 36-\mathrm{C} 37$ & $120.7(3)$ \\
\hline $\mathrm{C} 6-\mathrm{C} 7-\mathrm{H} 7$ & 119.7 & $\mathrm{C} 35-\mathrm{C} 36-\mathrm{H} 36$ & 119.7 \\
\hline $\mathrm{C} 7-\mathrm{C} 8-\mathrm{C} 8 \mathrm{~A}$ & $120.2(3)$ & $\mathrm{C} 37-\mathrm{C} 36-\mathrm{H} 36$ & 119.7 \\
\hline $\mathrm{C} 7-\mathrm{C} 8-\mathrm{H} 8$ & 119.9 & $\mathrm{C} 38-\mathrm{C} 37-\mathrm{C} 36$ & $119.8(3)$ \\
\hline $\mathrm{C} 8 \mathrm{~A}-\mathrm{C} 8-\mathrm{H} 8$ & 119.9 & $\mathrm{C} 38-\mathrm{C} 37-\mathrm{H} 37$ & 120.1 \\
\hline
\end{tabular}




\begin{tabular}{|c|c|c|c|}
\hline $\mathrm{N} 1-\mathrm{C} 8 \mathrm{~A}-\mathrm{C} 8$ & $119.2(2)$ & $\mathrm{C} 36-\mathrm{C} 37-\mathrm{H} 37$ & 120.1 \\
\hline $\mathrm{N} 1-\mathrm{C} 8 \mathrm{~A}-\mathrm{C} 4 \mathrm{~A}$ & $121.3(2)$ & $\mathrm{C} 37-\mathrm{C} 38-\mathrm{C} 38 \mathrm{~A}$ & $120.5(2)$ \\
\hline $\mathrm{C} 8-\mathrm{C} 8 \mathrm{~A}-\mathrm{C} 4 \mathrm{~A}$ & $119.5(2)$ & $\mathrm{C} 37-\mathrm{C} 38-\mathrm{H} 38$ & 119.7 \\
\hline $\mathrm{O} 31-\mathrm{C} 37 \mathrm{~A}-\mathrm{C} 3$ & $108.1(2)$ & $\mathrm{C} 38 \mathrm{~A}-\mathrm{C} 38-\mathrm{H} 38$ & 119.7 \\
\hline $\mathrm{O} 31-\mathrm{C} 37 \mathrm{~A}-\mathrm{H} 37 \mathrm{~A}$ & 110.1 & $\mathrm{C} 38-\mathrm{C} 38 \mathrm{~A}-\mathrm{C} 34 \mathrm{~A}$ & $119.5(2)$ \\
\hline $\mathrm{C} 3-\mathrm{C} 37 \mathrm{~A}-\mathrm{H} 37 \mathrm{~A}$ & 110.1 & $\mathrm{C} 38-\mathrm{C} 38 \mathrm{~A}-\mathrm{C} 31$ & $122.2(2)$ \\
\hline $\mathrm{O} 31-\mathrm{C} 37 \mathrm{~A}-\mathrm{H} 37 \mathrm{~B}$ & 110.1 & $\mathrm{C} 34 \mathrm{~A}-\mathrm{C} 38 \mathrm{~A}-\mathrm{C} 31$ & $118.2(2)$ \\
\hline $\mathrm{C} 3-\mathrm{C} 37 \mathrm{~A}-\mathrm{H} 37 \mathrm{~B}$ & 110.1 & & \\
\hline $\mathrm{C} 8 \mathrm{~A}-\mathrm{N} 1-\mathrm{C} 2-\mathrm{C} 3$ & $0.0(4)$ & $\mathrm{C} 3-\mathrm{C} 37 \mathrm{~A}-\mathrm{O} 31-\mathrm{C} 31$ & $175.4(2)$ \\
\hline $\mathrm{C} 8 \mathrm{~A}-\mathrm{N} 1-\mathrm{C} 2-\mathrm{Cl} 2$ & $179.65(19)$ & $\mathrm{C} 37 \mathrm{~A}-\mathrm{O} 31-\mathrm{C} 31-\mathrm{C} 32$ & $-1.4(4)$ \\
\hline $\mathrm{N} 1-\mathrm{C} 2-\mathrm{C} 3-\mathrm{C} 4$ & $-0.5(4)$ & $\mathrm{C} 37 \mathrm{~A}-\mathrm{O} 31-\mathrm{C} 31-\mathrm{C} 38 \mathrm{~A}$ & $177.9(2)$ \\
\hline $\mathrm{C} 12-\mathrm{C} 2-\mathrm{C} 3-\mathrm{C} 4$ & $179.83(19)$ & $\mathrm{O} 31-\mathrm{C} 31-\mathrm{C} 32-\mathrm{C} 33$ & $179.2(3)$ \\
\hline $\mathrm{N} 1-\mathrm{C} 2-\mathrm{C} 3-\mathrm{C} 37 \mathrm{~A}$ & $177.5(2)$ & $\mathrm{C} 38 \mathrm{~A}-\mathrm{C} 31-\mathrm{C} 32-\mathrm{C} 33$ & $-0.1(4)$ \\
\hline $\mathrm{Cl} 2-\mathrm{C} 2-\mathrm{C} 3-\mathrm{C} 37 \mathrm{~A}$ & $-2.1(3)$ & $\mathrm{C} 31-\mathrm{C} 32-\mathrm{C} 33-\mathrm{C} 34$ & $-1.7(4)$ \\
\hline $\mathrm{C} 2-\mathrm{C} 3-\mathrm{C} 4-\mathrm{C} 4 \mathrm{~A}$ & $0.7(4)$ & $\mathrm{C} 32-\mathrm{C} 33-\mathrm{C} 34-\mathrm{C} 34 \mathrm{~A}$ & $2.1(4)$ \\
\hline $\mathrm{C} 37 \mathrm{~A}-\mathrm{C} 3-\mathrm{C} 4-\mathrm{C} 4 \mathrm{~A}$ & $-177.3(2)$ & $\mathrm{C} 33-\mathrm{C} 34-\mathrm{C} 34 \mathrm{~A}-\mathrm{C} 35$ & $-179.0(2)$ \\
\hline $\mathrm{C} 3-\mathrm{C} 4-\mathrm{C} 4 \mathrm{~A}-\mathrm{C} 5$ & $178.4(3)$ & $\mathrm{C} 33-\mathrm{C} 34-\mathrm{C} 34 \mathrm{~A}-\mathrm{C} 38 \mathrm{~A}$ & $-0.7(4)$ \\
\hline $\mathrm{C} 3-\mathrm{C} 4-\mathrm{C} 4 \mathrm{~A}-\mathrm{C} 8 \mathrm{~A}$ & $-0.5(4)$ & $\mathrm{C} 34-\mathrm{C} 34 \mathrm{~A}-\mathrm{C} 35-\mathrm{C} 36$ & $179.7(3)$ \\
\hline $\mathrm{C} 4-\mathrm{C} 4 \mathrm{~A}-\mathrm{C} 5-\mathrm{C} 6$ & $-177.7(3)$ & $\mathrm{C} 38 \mathrm{~A}-\mathrm{C} 34 \mathrm{~A}-\mathrm{C} 35-\mathrm{C} 36$ & $1.4(4)$ \\
\hline $\mathrm{C} 8 \mathrm{~A}-\mathrm{C} 4 \mathrm{~A}-\mathrm{C} 5-\mathrm{C} 6$ & $1.2(4)$ & $\mathrm{C} 34 \mathrm{~A}-\mathrm{C} 35-\mathrm{C} 36-\mathrm{C} 37$ & $-0.6(4)$ \\
\hline $\mathrm{C} 4 \mathrm{~A}-\mathrm{C} 5-\mathrm{C} 6-\mathrm{C} 7$ & $-1.7(4)$ & $\mathrm{C} 35-\mathrm{C} 36-\mathrm{C} 37-\mathrm{C} 38$ & $0.3(4)$ \\
\hline $\mathrm{C} 5-\mathrm{C} 6-\mathrm{C} 7-\mathrm{C} 8$ & $0.7(5)$ & $\mathrm{C} 36-\mathrm{C} 37-\mathrm{C} 38-\mathrm{C} 38 \mathrm{~A}$ & $-0.9(4)$ \\
\hline $\mathrm{C} 6-\mathrm{C} 7-\mathrm{C} 8-\mathrm{C} 8 \mathrm{~A}$ & $0.9(4)$ & $\mathrm{C} 37-\mathrm{C} 38-\mathrm{C} 38 \mathrm{~A}-\mathrm{C} 34 \mathrm{~A}$ & $1.8(4)$ \\
\hline $\mathrm{C} 2-\mathrm{N} 1-\mathrm{C} 8 \mathrm{~A}-\mathrm{C} 8$ & $-179.1(2)$ & $\mathrm{C} 37-\mathrm{C} 38-\mathrm{C} 38 \mathrm{~A}-\mathrm{C} 31$ & $-177.5(2)$ \\
\hline $\mathrm{C} 2-\mathrm{N} 1-\mathrm{C} 8 \mathrm{~A}-\mathrm{C} 4 \mathrm{~A}$ & $0.3(4)$ & $\mathrm{C} 35-\mathrm{C} 34 \mathrm{~A}-\mathrm{C} 38 \mathrm{~A}-\mathrm{C} 38$ & $-2.0(4)$ \\
\hline $\mathrm{C} 7-\mathrm{C} 8-\mathrm{C} 8 \mathrm{~A}-\mathrm{N} 1$ & $178.0(3)$ & $\mathrm{C} 34-\mathrm{C} 34 \mathrm{~A}-\mathrm{C} 38 \mathrm{~A}-\mathrm{C} 38$ & $179.6(2)$ \\
\hline $\mathrm{C} 7-\mathrm{C} 8-\mathrm{C} 8 \mathrm{~A}-\mathrm{C} 4 \mathrm{~A}$ & $-1.4(4)$ & $\mathrm{C} 35-\mathrm{C} 34 \mathrm{~A}-\mathrm{C} 38 \mathrm{~A}-\mathrm{C} 31$ & $177.3(2)$ \\
\hline $\mathrm{C} 4-\mathrm{C} 4 \mathrm{~A}-\mathrm{C} 8 \mathrm{~A}-\mathrm{N} 1$ & $-0.1(4)$ & $\mathrm{C} 34-\mathrm{C} 34 \mathrm{~A}-\mathrm{C} 38 \mathrm{~A}-\mathrm{C} 31$ & $-1.0(3)$ \\
\hline $\mathrm{C} 5-\mathrm{C} 4 \mathrm{~A}-\mathrm{C} 8 \mathrm{~A}-\mathrm{N} 1$ & $-179.0(2)$ & $\mathrm{O} 31-\mathrm{C} 31-\mathrm{C} 38 \mathrm{~A}-\mathrm{C} 38$ & $1.4(3)$ \\
\hline $\mathrm{C} 4-\mathrm{C} 4 \mathrm{~A}-\mathrm{C} 8 \mathrm{~A}-\mathrm{C} 8$ & $179.3(2)$ & $\mathrm{C} 32-\mathrm{C} 31-\mathrm{C} 38 \mathrm{~A}-\mathrm{C} 38$ & $-179.2(2)$ \\
\hline $\mathrm{C} 5-\mathrm{C} 4 \mathrm{~A}-\mathrm{C} 8 \mathrm{~A}-\mathrm{C} 8$ & $0.4(4)$ & $\mathrm{O} 31-\mathrm{C} 31-\mathrm{C} 38 \mathrm{~A}-\mathrm{C} 34 \mathrm{~A}$ & $-177.9(2)$ \\
\hline $\mathrm{C} 4-\mathrm{C} 3-\mathrm{C} 37 \mathrm{~A}-\mathrm{O} 31$ & $-1.1(3)$ & $\mathrm{C} 32-\mathrm{C} 31-\mathrm{C} 38 \mathrm{~A}-\mathrm{C} 34 \mathrm{~A}$ & $1.4(3)$ \\
\hline $\mathrm{C} 2-\mathrm{C} 3-\mathrm{C} 37 \mathrm{~A}-\mathrm{O} 31$ & $-179.0(2)$ & & \\
\hline
\end{tabular}

Hydrogen-bond geometry $\left(\AA,{ }^{\circ}\right)$

\begin{tabular}{lllll}
\hline$D-\mathrm{H} \cdots A$ & $D-\mathrm{H}$ & $\mathrm{H} \cdots A$ & $D \cdots A$ & $D-\mathrm{H}^{\cdots} \cdot A$ \\
\hline $\mathrm{C} 37 A-\mathrm{H} 37 A^{\cdots} C g 3^{\mathrm{i}}$ & 0.99 & 2.74 & $3.552(3)$ & 139 \\
\hline
\end{tabular}

Symmetry code: (i) $x-1, y, z$.

(V) \{5-[(2-Chloroquinolin-3-yl)methoxy]-4-(hydroxymethyl)-6-methyl-pyridin-3-yl\}methanol

Crystal data

$\mathrm{C}_{18} \mathrm{H}_{17} \mathrm{ClN}_{2} \mathrm{O}_{3}$

$M_{r}=344.79$

Monoclinic, $P 2{ }_{1} / n$

$a=9.7866(3) \AA$

$$
\begin{aligned}
& b=15.3336(4) \AA \\
& c=10.6570(3) \AA \\
& \beta=92.381(3)^{\circ} \\
& V=1597.85(8) \AA^{3}
\end{aligned}
$$


$Z=4$

$F(000)=720$

$D_{\mathrm{x}}=1.433 \mathrm{Mg} \mathrm{m}^{-3}$

$\mathrm{Cu} K \alpha$ radiation, $\lambda=1.54184 \AA$

Cell parameters from 3112 reflections

Data collection

Agilent Eos Gemini

diffractometer

Radiation source: Enhance $(\mathrm{Cu}) \mathrm{X}$-ray Source

$\omega$ scans

Absorption correction: multi-scan

(SADABS; Sheldrick, 2003)

$T_{\min }=0.375, T_{\max }=0.481$

9423 measured reflections

\section{Refinement}

Refinement on $F^{2}$

Least-squares matrix: full

$R\left[F^{2}>2 \sigma\left(F^{2}\right)\right]=0.045$

$w R\left(F^{2}\right)=0.127$

$S=1.05$

3112 reflections

219 parameters

0 restraints

Hydrogen site location: mixed $\theta=5.1-72.5^{\circ}$

$\mu=2.29 \mathrm{~mm}^{-1}$

$T=173 \mathrm{~K}$

Block, colourless

$0.42 \times 0.38 \times 0.32 \mathrm{~mm}$

3112 independent reflections

2764 reflections with $I>2 \sigma(I)$

$R_{\text {int }}=0.043$

$\theta_{\text {max }}=72.5^{\circ}, \theta_{\min }=5.1^{\circ}$

$h=-11 \rightarrow 9$

$k=-17 \rightarrow 18$

$l=-13 \rightarrow 12$

Special details

Geometry. All e.s.d.'s (except the e.s.d. in the dihedral angle between two 1.s. planes) are estimated using the full covariance matrix. The cell e.s.d.'s are taken into account individually in the estimation of e.s.d.'s in distances, angles and torsion angles; correlations between e.s.d.'s in cell parameters are only used when they are defined by crystal symmetry. An approximate (isotropic) treatment of cell e.s.d.'s is used for estimating e.s.d.'s involving 1.s. planes.

Fractional atomic coordinates and isotropic or equivalent isotropic displacement parameters $\left(\AA^{2}\right)$

\begin{tabular}{lllll}
\hline & $x$ & $y$ & $z$ & $U_{\text {iso }} / U_{\text {eq }}$ \\
\hline $\mathrm{N} 1$ & $0.14131(14)$ & $0.33261(9)$ & $0.62337(13)$ & $0.0316(3)$ \\
$\mathrm{C} 2$ & $0.16152(16)$ & $0.41138(10)$ & $0.58407(15)$ & $0.0285(3)$ \\
$\mathrm{C} 2$ & $0.10286(4)$ & $0.49427(3)$ & $0.68208(4)$ & $0.03973(18)$ \\
$\mathrm{C} 3$ & $0.22557(16)$ & $0.43634(10)$ & $0.47234(16)$ & $0.0289(3)$ \\
$\mathrm{C} 4$ & $0.27768(17)$ & $0.36931(11)$ & $0.40445(15)$ & $0.0317(4)$ \\
$\mathrm{H} 4$ & 0.3241 & 0.3818 & 0.3300 & $0.038^{*}$ \\
$\mathrm{C} 4 \mathrm{~A}$ & $0.26375(17)$ & $0.28188(11)$ & $0.44305(16)$ & $0.0310(4)$ \\
$\mathrm{C} 5$ & $0.3168(2)$ & $0.21043(12)$ & $0.37582(18)$ & $0.0389(4)$ \\
$\mathrm{H} 5$ & 0.3677 & 0.2203 & 0.3031 & $0.047^{*}$ \\
$\mathrm{C} 6$ & $0.2941(2)$ & $0.12723(12)$ & $0.41650(19)$ & $0.0429(5)$ \\
$\mathrm{H} 6$ & 0.3305 & 0.0795 & 0.3720 & $0.052^{*}$ \\
$\mathrm{C} 7$ & $0.2183(2)$ & $0.11136(12)$ & $0.52249(19)$ & $0.0463(5)$ \\
$\mathrm{H} 7$ & 0.2022 & 0.0530 & 0.5479 & $0.056^{*}$ \\
$\mathrm{C} 8$ & $0.1668(2)$ & $0.17907(12)$ & $0.59013(17)$ & $0.0408(4)$ \\
$\mathrm{H} 8$ & 0.1155 & 0.1677 & 0.6621 & $0.049^{*}$ \\
$\mathrm{C} 8 \mathrm{~A}$ & $0.19062(18)$ & $0.26579(10)$ & $0.55190(16)$ & $0.0313(4)$
\end{tabular}

$\mathrm{H}$-atom parameters constrained

$w=1 /\left[\sigma^{2}\left(F_{\mathrm{o}}^{2}\right)+(0.0793 P)^{2}+0.2783 P\right]$ where $P=\left(F_{\mathrm{o}}^{2}+2 F_{\mathrm{c}}^{2}\right) / 3$

$(\Delta / \sigma)_{\max }=0.001$

$\Delta \rho_{\max }=0.32$ e $\AA^{-3}$

$\Delta \rho_{\min }=-0.25$ e $\AA^{-3}$

Extinction correction: SHELXL2014 (Sheldrick, 2015), $\mathrm{Fc}^{*}=\mathrm{kFc}\left[1+0.001 \mathrm{xFc}^{2} \lambda^{3} / \sin (2 \theta)\right]^{-1 / 4}$

Extinction coefficient: 0.0022 (4) 


$\begin{array}{lllll}\text { C37 } & 0.23683(17) & 0.52862(11) & 0.42748(17) & 0.0329(4) \\ \text { H37A } & 0.2336 & 0.5302 & 0.3345 & 0.039^{*} \\ \text { H37B } & 0.1596 & 0.5636 & 0.4573 & 0.039^{*} \\ \text { O31 } & 0.36442(11) & 0.56428(7) & 0.47603(10) & 0.0276(3) \\ \text { N31 } & 0.51999(13) & 0.70720(9) & 0.25268(13) & 0.0297(3) \\ \text { C32 } & 0.47930(15) & 0.63485(10) & 0.30994(15) & 0.0259(3) \\ \text { C33 } & 0.39991(15) & 0.64051(10) & 0.41634(14) & 0.0236(3) \\ \text { C34 } & 0.36223(15) & 0.72095(10) & 0.46347(14) & 0.0258(3) \\ \text { C35 } & 0.40670(16) & 0.79604(10) & 0.40161(15) & 0.0284(3) \\ \text { C36 } & 0.48433(17) & 0.78469(10) & 0.29806(16) & 0.0311(4) \\ \text { H36 } & 0.5145 & 0.8354 & 0.2561 & 0.037^{*} \\ \text { C321 } & 0.52007(17) & 0.54887(11) & 0.25592(17) & 0.0329(4) \\ \text { H32A } & 0.5987 & 0.5572 & 0.2032 & 0.039^{*} \\ \text { H32B } & 0.4434 & 0.5249 & 0.2049 & 0.039^{*} \\ \text { H32C } & 0.5447 & 0.5084 & 0.3243 & 0.039^{*} \\ \text { C341 } & 0.27452(17) & 0.72795(11) & 0.57607(15) & 0.0316(4) \\ \text { H41A } & 0.2588 & 0.6694 & 0.6119 & 0.038^{*} \\ \text { H41B } & 0.3204 & 0.7647 & 0.6416 & 0.038^{*} \\ \text { O341 } & 0.14772(13) & 0.76632(10) & 0.53514(12) & 0.0437(3) \\ \text { H341 } & 0.1027 & 0.7791 & 0.6056 & 0.066^{*} \\ \text { C351 } & 0.36913(19) & 0.88673(11) & 0.44319(18) & 0.0371(4) \\ \text { H51A } & 0.3812 & 0.8906 & 0.5357 & 0.045^{*} \\ \text { H51B } & 0.4321 & 0.9293 & 0.4063 & 0.045^{*} \\ \text { O351 } & 0.23190(14) & 0.90946(8) & 0.40706(13) & 0.0434(3) \\ \text { H351 } & 0.1838 & 0.8642 & 0.4382 & 0.065^{*}\end{array}$

Atomic displacement parameters $\left(\AA^{2}\right)$

\begin{tabular}{lllllll}
\hline & $U^{11}$ & $U^{22}$ & $U^{33}$ & $U^{12}$ & $U^{13}$ & $U^{23}$ \\
\hline N1 & $0.0401(7)$ & $0.0246(7)$ & $0.0303(7)$ & $-0.0041(5)$ & $0.0016(6)$ & $0.0012(5)$ \\
C2 & $0.0320(7)$ & $0.0220(7)$ & $0.0314(8)$ & $-0.0007(6)$ & $0.0000(6)$ & $-0.0026(6)$ \\
C12 & $0.0436(3)$ & $0.0287(2)$ & $0.0476(3)$ & $0.00182(15)$ & $0.0100(2)$ & $-0.00770(16)$ \\
C3 & $0.0312(8)$ & $0.0235(8)$ & $0.0318(8)$ & $-0.0028(6)$ & $-0.0022(6)$ & $0.0030(6)$ \\
C4 & $0.0380(8)$ & $0.0295(9)$ & $0.0274(8)$ & $-0.0038(6)$ & $0.0000(6)$ & $0.0017(6)$ \\
C4A & $0.0377(8)$ & $0.0258(8)$ & $0.0290(8)$ & $-0.0007(6)$ & $-0.0044(7)$ & $-0.0014(6)$ \\
C5 & $0.0460(10)$ & $0.0349(9)$ & $0.0353(9)$ & $0.0023(7)$ & $-0.0043(8)$ & $-0.0076(7)$ \\
C6 & $0.0575(11)$ & $0.0270(9)$ & $0.0430(10)$ & $0.0059(8)$ & $-0.0132(9)$ & $-0.0105(7)$ \\
C7 & $0.0708(13)$ & $0.0214(8)$ & $0.0453(11)$ & $-0.0039(8)$ & $-0.0149(10)$ & $-0.0006(7)$ \\
C8 & $0.0602(11)$ & $0.0266(9)$ & $0.0350(9)$ & $-0.0081(8)$ & $-0.0051(8)$ & $0.0024(7)$ \\
C8A & $0.0413(9)$ & $0.0233(8)$ & $0.0285(8)$ & $-0.0029(6)$ & $-0.0068(7)$ & $0.0009(6)$ \\
C37 & $0.0346(8)$ & $0.0255(8)$ & $0.0381(9)$ & $-0.0030(6)$ & $-0.0039(7)$ & $0.0081(7)$ \\
O31 & $0.0319(6)$ & $0.0216(5)$ & $0.0292(6)$ & $-0.0012(4)$ & $0.0000(4)$ & $0.0060(4)$ \\
N31 & $0.0299(7)$ & $0.0274(7)$ & $0.0324(7)$ & $-0.0012(5)$ & $0.0082(5)$ & $0.0014(5)$ \\
C32 & $0.0255(7)$ & $0.0240(8)$ & $0.0280(8)$ & $0.0017(6)$ & $0.0018(6)$ & $-0.0011(6)$ \\
C33 & $0.0254(7)$ & $0.0204(7)$ & $0.0251(7)$ & $-0.0004(5)$ & $0.0005(6)$ & $0.0025(5)$ \\
C34 & $0.0284(7)$ & $0.0250(8)$ & $0.0240(7)$ & $0.0007(5)$ & $0.0012(6)$ & $-0.0002(6)$ \\
C35 & $0.0333(8)$ & $0.0201(7)$ & $0.0318(8)$ & $-0.0005(6)$ & $0.0025(6)$ & $-0.0011(6)$ \\
C36 & $0.0331(8)$ & $0.0256(8)$ & $0.0351(9)$ & $-0.0033(6)$ & $0.0064(6)$ & $0.0038(6)$
\end{tabular}




\begin{tabular}{lllllll} 
C321 & $0.0363(8)$ & $0.0274(8)$ & $0.0354(9)$ & $0.0056(6)$ & $0.0058(7)$ & $-0.0048(7)$ \\
C341 & $0.0407(9)$ & $0.0304(8)$ & $0.0244(8)$ & $0.0032(6)$ & $0.0084(6)$ & $-0.0011(6)$ \\
O341 & $0.0404(7)$ & $0.0575(8)$ & $0.0343(7)$ & $0.0124(6)$ & $0.0145(5)$ & $0.0018(6)$ \\
C351 & $0.0481(10)$ & $0.0220(8)$ & $0.0415(10)$ & $0.0018(7)$ & $0.0067(8)$ & $-0.0035(7)$ \\
O351 & $0.0531(8)$ & $0.0307(7)$ & $0.0467(8)$ & $0.0135(6)$ & $0.0046(6)$ & $-0.0013(5)$ \\
\hline
\end{tabular}

Geometric parameters $\left(\hat{A},{ }^{\circ}\right)$

\begin{tabular}{|c|c|c|c|}
\hline $\mathrm{N} 1-\mathrm{C} 2$ & $1.296(2)$ & N31-C36 & $1.335(2)$ \\
\hline $\mathrm{N} 1-\mathrm{C} 8 \mathrm{~A}$ & $1.376(2)$ & $\mathrm{N} 31-\mathrm{C} 32$ & $1.335(2)$ \\
\hline $\mathrm{C} 2-\mathrm{C} 3$ & $1.421(2)$ & $\mathrm{C} 32-\mathrm{C} 33$ & $1.404(2)$ \\
\hline $\mathrm{C} 2-\mathrm{Cl} 2$ & $1.7566(16)$ & $\mathrm{C} 32-\mathrm{C} 321$ & $1.499(2)$ \\
\hline $\mathrm{C} 3-\mathrm{C} 4$ & $1.368(2)$ & $\mathrm{C} 33-\mathrm{C} 34$ & $1.388(2)$ \\
\hline $\mathrm{C} 3-\mathrm{C} 37$ & $1.499(2)$ & $\mathrm{C} 34-\mathrm{C} 35$ & $1.405(2)$ \\
\hline $\mathrm{C} 4-\mathrm{C} 4 \mathrm{~A}$ & $1.411(2)$ & $\mathrm{C} 34-\mathrm{C} 341$ & $1.508(2)$ \\
\hline $\mathrm{C} 4-\mathrm{H} 4$ & 0.9500 & $\mathrm{C} 35-\mathrm{C} 36$ & $1.377(2)$ \\
\hline $\mathrm{C} 4 \mathrm{~A}-\mathrm{C} 8 \mathrm{~A}$ & $1.410(2)$ & $\mathrm{C} 35-\mathrm{C} 351$ & $1.510(2)$ \\
\hline $\mathrm{C} 4 \mathrm{~A}-\mathrm{C} 5$ & $1.419(2)$ & $\mathrm{C} 36-\mathrm{H} 36$ & 0.9500 \\
\hline $\mathrm{C} 5-\mathrm{C} 6$ & $1.368(3)$ & C321-H32A & 0.9800 \\
\hline $\mathrm{C} 5-\mathrm{H} 5$ & 0.9500 & C321-H32B & 0.9800 \\
\hline $\mathrm{C} 6-\mathrm{C} 7$ & $1.398(3)$ & $\mathrm{C} 321-\mathrm{H} 32 \mathrm{C}$ & 0.9800 \\
\hline $\mathrm{C} 6-\mathrm{H} 6$ & 0.9500 & $\mathrm{C} 341-\mathrm{O} 341$ & $1.425(2)$ \\
\hline $\mathrm{C} 7-\mathrm{C} 8$ & $1.372(3)$ & C341-H41A & 0.9900 \\
\hline $\mathrm{C} 7-\mathrm{H} 7$ & 0.9500 & $\mathrm{C} 341-\mathrm{H} 41 \mathrm{~B}$ & 0.9900 \\
\hline $\mathrm{C} 8-\mathrm{C} 8 \mathrm{~A}$ & $1.413(2)$ & $\mathrm{O} 341-\mathrm{H} 341$ & 0.9077 \\
\hline $\mathrm{C} 8-\mathrm{H} 8$ & 0.9500 & $\mathrm{C} 351-\mathrm{O} 351$ & $1.425(2)$ \\
\hline $\mathrm{C} 37-\mathrm{O} 31$ & $1.4394(19)$ & C351-H51A & 0.9900 \\
\hline C37-H37A & 0.9900 & C351-H51B & 0.9900 \\
\hline C37-H37B & 0.9900 & O351-H351 & 0.9093 \\
\hline $\mathrm{O} 31-\mathrm{C} 33$ & $1.3819(18)$ & & \\
\hline $\mathrm{C} 2-\mathrm{N} 1-\mathrm{C} 8 \mathrm{~A}$ & $116.95(14)$ & $\mathrm{N} 31-\mathrm{C} 32-\mathrm{C} 33$ & $120.23(14)$ \\
\hline $\mathrm{N} 1-\mathrm{C} 2-\mathrm{C} 3$ & $126.88(15)$ & $\mathrm{N} 31-\mathrm{C} 32-\mathrm{C} 321$ & $117.78(14)$ \\
\hline $\mathrm{N} 1-\mathrm{C} 2-\mathrm{Cl} 2$ & $115.09(12)$ & $\mathrm{C} 33-\mathrm{C} 32-\mathrm{C} 321$ & $121.98(14)$ \\
\hline $\mathrm{C} 3-\mathrm{C} 2-\mathrm{Cl} 2$ & $118.02(12)$ & $\mathrm{O} 31-\mathrm{C} 33-\mathrm{C} 34$ & $120.64(14)$ \\
\hline $\mathrm{C} 4-\mathrm{C} 3-\mathrm{C} 2$ & $115.34(14)$ & $\mathrm{O} 31-\mathrm{C} 33-\mathrm{C} 32$ & $118.54(13)$ \\
\hline $\mathrm{C} 4-\mathrm{C} 3-\mathrm{C} 37$ & $120.45(15)$ & $\mathrm{C} 34-\mathrm{C} 33-\mathrm{C} 32$ & $120.78(13)$ \\
\hline $\mathrm{C} 2-\mathrm{C} 3-\mathrm{C} 37$ & $124.21(15)$ & $\mathrm{C} 33-\mathrm{C} 34-\mathrm{C} 35$ & $117.81(14)$ \\
\hline $\mathrm{C} 3-\mathrm{C} 4-\mathrm{C} 4 \mathrm{~A}$ & $121.15(15)$ & $\mathrm{C} 33-\mathrm{C} 34-\mathrm{C} 341$ & $121.33(14)$ \\
\hline $\mathrm{C} 3-\mathrm{C} 4-\mathrm{H} 4$ & 119.4 & $\mathrm{C} 35-\mathrm{C} 34-\mathrm{C} 341$ & $120.85(14)$ \\
\hline $\mathrm{C} 4 \mathrm{~A}-\mathrm{C} 4-\mathrm{H} 4$ & 119.4 & $\mathrm{C} 36-\mathrm{C} 35-\mathrm{C} 34$ & $117.68(14)$ \\
\hline $\mathrm{C} 8 \mathrm{~A}-\mathrm{C} 4 \mathrm{~A}-\mathrm{C} 4$ & $117.69(15)$ & $\mathrm{C} 36-\mathrm{C} 35-\mathrm{C} 351$ & $120.08(15)$ \\
\hline $\mathrm{C} 8 \mathrm{~A}-\mathrm{C} 4 \mathrm{~A}-\mathrm{C} 5$ & $119.29(15)$ & $\mathrm{C} 34-\mathrm{C} 35-\mathrm{C} 351$ & $122.22(15)$ \\
\hline $\mathrm{C} 4-\mathrm{C} 4 \mathrm{~A}-\mathrm{C} 5$ & $123.00(16)$ & $\mathrm{N} 31-\mathrm{C} 36-\mathrm{C} 35$ & $124.36(14)$ \\
\hline $\mathrm{C} 6-\mathrm{C} 5-\mathrm{C} 4 \mathrm{~A}$ & $119.48(18)$ & $\mathrm{N} 31-\mathrm{C} 36-\mathrm{H} 36$ & 117.8 \\
\hline $\mathrm{C} 6-\mathrm{C} 5-\mathrm{H} 5$ & 120.3 & $\mathrm{C} 35-\mathrm{C} 36-\mathrm{H} 36$ & 117.8 \\
\hline $\mathrm{C} 4 \mathrm{~A}-\mathrm{C} 5-\mathrm{H} 5$ & 120.3 & $\mathrm{C} 32-\mathrm{C} 321-\mathrm{H} 32 \mathrm{~A}$ & 109.5 \\
\hline $\mathrm{C} 5-\mathrm{C} 6-\mathrm{C} 7$ & $121.11(17)$ & $\mathrm{C} 32-\mathrm{C} 321-\mathrm{H} 32 \mathrm{~B}$ & 109.5 \\
\hline
\end{tabular}




\begin{tabular}{|c|c|}
\hline $\mathrm{C} 5-\mathrm{C} 6-\mathrm{H} 6$ & 119.4 \\
\hline $\mathrm{C} 7-\mathrm{C} 6-\mathrm{H} 6$ & 119.4 \\
\hline $\mathrm{C} 8-\mathrm{C} 7-\mathrm{C} 6$ & $120.77(17)$ \\
\hline $\mathrm{C} 8-\mathrm{C} 7-\mathrm{H} 7$ & 119.6 \\
\hline $\mathrm{C} 6-\mathrm{C} 7-\mathrm{H} 7$ & 119.6 \\
\hline $\mathrm{C} 7-\mathrm{C} 8-\mathrm{C} 8 \mathrm{~A}$ & $119.49(18)$ \\
\hline $\mathrm{C} 7-\mathrm{C} 8-\mathrm{H} 8$ & 120.3 \\
\hline $\mathrm{C} 8 \mathrm{~A}-\mathrm{C} 8-\mathrm{H} 8$ & 120.3 \\
\hline $\mathrm{N} 1-\mathrm{C} 8 \mathrm{~A}-\mathrm{C} 4 \mathrm{~A}$ & $121.79(15)$ \\
\hline $\mathrm{N} 1-\mathrm{C} 8 \mathrm{~A}-\mathrm{C} 8$ & $118.38(16)$ \\
\hline $\mathrm{C} 4 \mathrm{~A}-\mathrm{C} 8 \mathrm{~A}-\mathrm{C} 8$ & $119.83(16)$ \\
\hline $\mathrm{O} 31-\mathrm{C} 37-\mathrm{C} 3$ & $108.55(12)$ \\
\hline $\mathrm{O} 31-\mathrm{C} 37-\mathrm{H} 37 \mathrm{~A}$ & 110.0 \\
\hline $\mathrm{C} 3-\mathrm{C} 37-\mathrm{H} 37 \mathrm{~A}$ & 110.0 \\
\hline $\mathrm{O} 31-\mathrm{C} 37-\mathrm{H} 37 \mathrm{~B}$ & 110.0 \\
\hline $\mathrm{C} 3-\mathrm{C} 37-\mathrm{H} 37 \mathrm{~B}$ & 110.0 \\
\hline $\mathrm{H} 37 \mathrm{~A}-\mathrm{C} 37-\mathrm{H} 37 \mathrm{~B}$ & 108.4 \\
\hline $\mathrm{C} 33-\mathrm{O} 31-\mathrm{C} 37$ & $112.75(11)$ \\
\hline $\mathrm{C} 36-\mathrm{N} 31-\mathrm{C} 32$ & $119.13(14)$ \\
\hline $\mathrm{C} 8 \mathrm{~A}-\mathrm{N} 1-\mathrm{C} 2-\mathrm{C} 3$ & $2.2(2)$ \\
\hline $\mathrm{C} 8 \mathrm{~A}-\mathrm{N} 1-\mathrm{C} 2-\mathrm{Cl} 2$ & $-177.48(11)$ \\
\hline $\mathrm{N} 1-\mathrm{C} 2-\mathrm{C} 3-\mathrm{C} 4$ & $-4.1(2)$ \\
\hline $\mathrm{Cl} 2-\mathrm{C} 2-\mathrm{C} 3-\mathrm{C} 4$ & $175.57(12)$ \\
\hline $\mathrm{N} 1-\mathrm{C} 2-\mathrm{C} 3-\mathrm{C} 37$ & $175.40(15)$ \\
\hline $\mathrm{C} 12-\mathrm{C} 2-\mathrm{C} 3-\mathrm{C} 37$ & $-4.9(2)$ \\
\hline $\mathrm{C} 2-\mathrm{C} 3-\mathrm{C} 4-\mathrm{C} 4 \mathrm{~A}$ & $1.8(2)$ \\
\hline $\mathrm{C} 37-\mathrm{C} 3-\mathrm{C} 4-\mathrm{C} 4 \mathrm{~A}$ & $-177.78(14)$ \\
\hline $\mathrm{C} 3-\mathrm{C} 4-\mathrm{C} 4 \mathrm{~A}-\mathrm{C} 8 \mathrm{~A}$ & $1.9(2)$ \\
\hline $\mathrm{C} 3-\mathrm{C} 4-\mathrm{C} 4 \mathrm{~A}-\mathrm{C} 5$ & $-179.68(15)$ \\
\hline $\mathrm{C} 8 \mathrm{~A}-\mathrm{C} 4 \mathrm{~A}-\mathrm{C} 5-\mathrm{C} 6$ & $1.1(3)$ \\
\hline $\mathrm{C} 4-\mathrm{C} 4 \mathrm{~A}-\mathrm{C} 5-\mathrm{C} 6$ & $-177.29(16)$ \\
\hline $\mathrm{C} 4 \mathrm{~A}-\mathrm{C} 5-\mathrm{C} 6-\mathrm{C} 7$ & $0.7(3)$ \\
\hline $\mathrm{C} 5-\mathrm{C} 6-\mathrm{C} 7-\mathrm{C} 8$ & $-1.3(3)$ \\
\hline $\mathrm{C} 6-\mathrm{C} 7-\mathrm{C} 8-\mathrm{C} 8 \mathrm{~A}$ & $0.1(3)$ \\
\hline $\mathrm{C} 2-\mathrm{N} 1-\mathrm{C} 8 \mathrm{~A}-\mathrm{C} 4 \mathrm{~A}$ & $2.0(2)$ \\
\hline $\mathrm{C} 2-\mathrm{N} 1-\mathrm{C} 8 \mathrm{~A}-\mathrm{C} 8$ & $-178.19(15)$ \\
\hline $\mathrm{C} 4-\mathrm{C} 4 \mathrm{~A}-\mathrm{C} 8 \mathrm{~A}-\mathrm{N} 1$ & $-4.0(2)$ \\
\hline $\mathrm{C} 5-\mathrm{C} 4 \mathrm{~A}-\mathrm{C} 8 \mathrm{~A}-\mathrm{N} 1$ & $177.53(15)$ \\
\hline $\mathrm{C} 4-\mathrm{C} 4 \mathrm{~A}-\mathrm{C} 8 \mathrm{~A}-\mathrm{C} 8$ & $176.21(15)$ \\
\hline $\mathrm{C} 5-\mathrm{C} 4 \mathrm{~A}-\mathrm{C} 8 \mathrm{~A}-\mathrm{C} 8$ & $-2.3(2)$ \\
\hline $\mathrm{C} 7-\mathrm{C} 8-\mathrm{C} 8 \mathrm{~A}-\mathrm{N} 1$ & $-178.14(16)$ \\
\hline $\mathrm{C} 7-\mathrm{C} 8-\mathrm{C} 8 \mathrm{~A}-\mathrm{C} 4 \mathrm{~A}$ & $1.7(3)$ \\
\hline $\mathrm{C} 4-\mathrm{C} 3-\mathrm{C} 37-\mathrm{O} 31$ & $-88.42(18)$ \\
\hline $\mathrm{C} 2-\mathrm{C} 3-\mathrm{C} 37-\mathrm{O} 31$ & $92.08(18)$ \\
\hline
\end{tabular}

$\mathrm{H} 32 \mathrm{~A}-\mathrm{C} 321-\mathrm{H} 32 \mathrm{~B} \quad 109.5$

$\mathrm{C} 32-\mathrm{C} 321-\mathrm{H} 32 \mathrm{C} \quad 109.5$

$\mathrm{H} 32 \mathrm{~A}-\mathrm{C} 321-\mathrm{H} 32 \mathrm{C} \quad 109.5$

$\mathrm{H} 32 \mathrm{~B}-\mathrm{C} 321-\mathrm{H} 32 \mathrm{C} \quad 109.5$

$\mathrm{O} 341-\mathrm{C} 341-\mathrm{C} 34 \quad 107.64$ (13)

O341-C341-H41A $\quad 110.2$

$\mathrm{C} 34-\mathrm{C} 341-\mathrm{H} 41 \mathrm{~A} \quad 110.2$

O341-C341-H41B $\quad 110.2$

$\mathrm{C} 34-\mathrm{C} 341-\mathrm{H} 41 \mathrm{~B} \quad 110.2$

$\mathrm{H} 41 \mathrm{~A}-\mathrm{C} 341-\mathrm{H} 41 \mathrm{~B} \quad 108.5$

C341-O341-H341 106.4

$\mathrm{O} 351-\mathrm{C} 351-\mathrm{C} 35 \quad 112.61(14)$

O351-C351-H51A $\quad 109.1$

C35-C351-H51A $\quad 109.1$

O351-C351-H51B $\quad 109.1$

$\mathrm{C} 35-\mathrm{C} 351-\mathrm{H} 51 \mathrm{~B} \quad 109.1$

$\mathrm{H} 51 \mathrm{~A}-\mathrm{C} 351-\mathrm{H} 51 \mathrm{~B} \quad 107.8$

C351-O351-H351 102.2

$\mathrm{C} 3-\mathrm{C} 37-\mathrm{O} 31-\mathrm{C} 33 \quad 165.21$ (13)

$\mathrm{C} 36-\mathrm{N} 31-\mathrm{C} 32-\mathrm{C} 33 \quad 0.1(2)$

$\mathrm{C} 36-\mathrm{N} 31-\mathrm{C} 32-\mathrm{C} 321$

$\mathrm{C} 37-\mathrm{O} 31-\mathrm{C} 33-\mathrm{C} 34 \quad 92.25$ (17)

$\mathrm{C} 37-\mathrm{O} 31-\mathrm{C} 33-\mathrm{C} 32 \quad-90.17$ (17)

$\mathrm{N} 31-\mathrm{C} 32-\mathrm{C} 33-\mathrm{O} 31 \quad-177.42$ (13)

$\mathrm{C} 321-\mathrm{C} 32-\mathrm{C} 33-\mathrm{O} 31 \quad 3.2(2)$

$\mathrm{N} 31-\mathrm{C} 32-\mathrm{C} 33-\mathrm{C} 34 \quad 0.2(2)$

$\mathrm{C} 321-\mathrm{C} 32-\mathrm{C} 33-\mathrm{C} 34 \quad-179.21(14)$

$\mathrm{O} 31-\mathrm{C} 33-\mathrm{C} 34-\mathrm{C} 35 \quad 177.22$ (13)

$\mathrm{C} 32-\mathrm{C} 33-\mathrm{C} 34-\mathrm{C} 35 \quad-0.3(2)$

$\mathrm{O} 31-\mathrm{C} 33-\mathrm{C} 34-\mathrm{C} 341 \quad-3.8(2)$

$\mathrm{C} 32-\mathrm{C} 33-\mathrm{C} 34-\mathrm{C} 341 \quad 178.71$ (14)

$\mathrm{C} 33-\mathrm{C} 34-\mathrm{C} 35-\mathrm{C} 36 \quad 0.2(2)$

$\mathrm{C} 341-\mathrm{C} 34-\mathrm{C} 35-\mathrm{C} 36 \quad-178.80$ (14)

$\mathrm{C} 33-\mathrm{C} 34-\mathrm{C} 35-\mathrm{C} 351 \quad 178.66$ (14)

$\mathrm{C} 341-\mathrm{C} 34-\mathrm{C} 35-\mathrm{C} 351 \quad-0.4$ (2)

$\mathrm{C} 32-\mathrm{N} 31-\mathrm{C} 36-\mathrm{C} 35-0.2(3)$

$\mathrm{C} 34-\mathrm{C} 35-\mathrm{C} 36-\mathrm{N} 31 \quad 0.0(3)$

$\mathrm{C} 351-\mathrm{C} 35-\mathrm{C} 36-\mathrm{N} 31 \quad-178.46(15)$

$\mathrm{C} 33-\mathrm{C} 34-\mathrm{C} 341-\mathrm{O} 341 \quad-114.67$ (16)

$\mathrm{C} 35-\mathrm{C} 34-\mathrm{C} 341-\mathrm{O} 341 \quad 64.32$ (19)

$\mathrm{C} 36-\mathrm{C} 35-\mathrm{C} 351-\mathrm{O} 351 \quad 102.07$ (19)

$\mathrm{C} 34-\mathrm{C} 35-\mathrm{C} 351-\mathrm{O} 351 \quad-76.3$ (2) 


\section{supporting information}

Hydrogen-bond geometry $\left(\AA,{ }^{\circ}\right)$

\begin{tabular}{lllll}
\hline$D-\mathrm{H} \cdots A$ & $D-\mathrm{H}$ & $\mathrm{H} \cdots A$ & $D \cdots A$ & $D-\mathrm{H} \cdots A$ \\
\hline $\mathrm{O} 341-\mathrm{H} 341 \cdots \mathrm{N} 31^{\mathrm{i}}$ & 0.91 & 1.81 & $2.7098(19)$ & 174 \\
$\mathrm{O} 351-\mathrm{H} 351 \cdots \mathrm{O} 341$ & 0.91 & 1.86 & $2.7299(19)$ & 158 \\
$\mathrm{C} 4-\mathrm{H} 4 \cdots \mathrm{O} 351^{\mathrm{ii}}$ & 0.95 & 2.60 & $3.374(2)$ & 139
\end{tabular}

Symmetry codes: (i) $x-1 / 2,-y+3 / 2, z+1 / 2$; (ii) $-x+1 / 2, y-1 / 2,-z+1 / 2$. 